\title{
Constant scalar curvature metrics with isolated singularities
}

\author{
Rafe Mazzeo* \\ Stanford University \\ mazzeo@math.stanford.edu
}

\author{
Frank Pacard \\ Universitè de Paris XII \\ Frank.Pacard@diam1.ens-cachan.fr
}

\begin{abstract}
We extend the results and methods of [6] to prove the existence of constant positive scalar curvature metrics $g$ which are complete and conformal to the standard metric on $S^{N} \backslash \Lambda$, where $\Lambda$ is a disjoint union of submanifolds of dimensions between 0 and $(N-2) / 2$. The existence of solutions with isolated singularities occupies the majority of the paper; their existence was previously established by Schoen [12], but the proof we give here, based on the techniques of [6], is more direct, and provides more information about their geometry. When $\Lambda$ is discrete we also establish that these solutions are smooth points in the moduli spaces of all such solutions introduced and studied in [7] and [8]
\end{abstract}

\section{Introduction and statement of the results}

In this paper we construct solutions of the Yamabe problem on the sphere $\left(S^{N}, g_{0}\right)$ with its standard metric which are singular at a specified closed set $\Lambda \subset S^{N}$. More specifically, we seek a new metric $g$ conformal to $g_{0}$ and complete on $\Lambda \subset S^{N}$, and with constant positive scalar curvature $R$. The problem may be translated into a differential equation as follows. Since $g$ is conformal to $g_{0}$, we may write $g=u^{\frac{4}{N-2}} g_{0}$ where $u$ is a positive function on $M \backslash \Lambda$. The scalar curvature functions $R\left(g_{0}\right)$ of $g_{0}$ and $R(g)$ of $g$ are related by the equation

$$
\Delta_{g_{0}} u-\frac{N-2}{4(N-1)} R\left(g_{0}\right) u+\frac{N-2}{4(N-1)} R(g) u^{\frac{N+2}{N-2}}=0 .
$$

In order that $g$ be complete on $S^{N} \backslash \Lambda$ it is necessary for $u$ to tend to infinity rather strongly on approach to $\Lambda$, and of course, we wish to solve this equation with $R$ a (prescribed) constant.

The first two terms of the operator on the left in (1), namely

$$
\mathcal{L}_{g_{0}} \equiv \Delta_{g_{0}}-\frac{N-2}{4(N-1)} R\left(g_{0}\right)
$$

give a second order linear elliptic differential operator known as the conformal Laplacian of the metric $g_{0}$. It satisfies the conformal equivariance property that if two metrics are conformally related, such as $g$ and $g_{0}$ above, then for any function $\phi$,

$$
\mathcal{L}_{g_{0}}(u \phi)=u^{\frac{N+2}{N-2}} \mathcal{L}_{g}(\phi) .
$$

Notice that (1) corresponds to the special case of (3) when $\phi=1$.

This 'singular Yamabe problem' has been extensively studied in recent years, also in the case when the ambient manifold is more general than the sphere, and many existence results as well as obstructions to existence are known. Briefly, for a solution to exist on a general compact

\footnotetext{
*Supported by the NSF with a Young Investigator Fellowship and by grant \#DMS-9303236
} 
Riemannian manifold $\left(M, g_{0}\right)$, the size of $\Lambda$ and the sign of $R$ must be related to one another : if a solution exists with $R<0$, then $\operatorname{dim}(\Lambda)>\frac{N-2}{2}$, while if a solution exists with $R \geq 0$, then $\operatorname{dim}(\Lambda) \leq \frac{N-2}{2}$ and in addition the first eigenvalue of the conformal Laplacian of $g_{0}$ must be nonnegative. Here the dimension is to be interpreted as Hausdorff dimension. Unfortunately, only partial converses to these statements are known. In particular, almost all of the existence results require $\Lambda$ to be a submanifold, or at worst a stratified set.

The character of the analysis required to prove existence of solutions when $R<0$ is quite different than in the other cases. In fact, it is not too difficult, using barrier methods, to construct solutions of (1). The difficulty is in showing that these solutions have a singularity at $\Lambda$ strong enough for $g$ to be complete on the complement. Similarly, when $R=0,(1)$ becomes linear, albeit of a somewhat nonstandard sort. We shall not discuss these cases any further, but refer to the work of R. McOwen [10], D. Finn [1], [2] and the first author [5], and references therein.

We shall concentrate here on the case $R>0$. The first examples of solutions were constructed by R. Schoen [12] when $\Lambda$ is either a finite set of points (of cardinality greater than one) or a nonrectifiable set arising essentially as the limit set of a Kleinian group. Nontrivial solutions with higher dimensional singular sets were constructed by the first author and N. Smale [9], the second author [11], and finally in some generality by both of us [6]. This last result states that solutions may be constructed on an arbitrary compact manifold of nonnegative scalar curvature $M$ whenever $\Lambda$ is a finite disjoint union of submanifolds of dimensions between 1 and $\frac{N-2}{2}$. This paper is meant to extend the methods of [6] to also allow for the construction of solutions with isolated singularities in the case where $M=S^{N}$ and $g_{0}$ is the standard metric on $S^{N}$, i.e. to allow $\Lambda$ to be an arbitrary disjoint finite union of submanifolds of dimensions between 0 and $\frac{N-2}{2}$. Our main result is:

Theorem 1 Suppose that $\Lambda$ is a disjoint union of submanifolds in $S^{N}$ of varying dimensions. Write $\Lambda=\Lambda^{\prime} \cup \Lambda^{\prime \prime}$, where $\Lambda^{\prime}$ is the union of all submanifolds of dimension zero, i.e. $\Lambda^{\prime}=$ $\left\{p_{1}, \ldots, p_{n}\right\}$ is a collection of points, and $\Lambda^{\prime \prime}=\bigcup_{j=1}^{n} \Lambda_{j}$ where $\operatorname{dim} \Lambda_{j}=k_{j}$. Suppose further that $0<k_{j} \leq \frac{N-2}{2}$ for each $j$, and either $n=0$ or $n \geq 2$. Then there exists a complete metric $g=u^{\frac{N+2}{N-2}} g_{0}$ on $S^{N} \backslash \Lambda$ which has constant positive scalar curvature $R=N(N-1)$.

For most of this paper we shall consider the case where $\Lambda^{\prime \prime}$ is empty, so that $\Lambda$ is a finite collection of points

$$
\Lambda=\left\{p_{1}, \ldots, p_{n}\right\} \subset S^{N} .
$$

The modifications needed to treat the general case are an amalgamation of the techniques here and those of [6], and shall be described in a brief final section of this paper.

Of course, solutions with isolated singularities were already constructed by R. Schoen, but his remarkable proof is long and difficult. We feel that (the extension of) the methods of [6] avoid many of the difficulties he encountered and substantially clarify the analysis. In addition, various properties of the solutions follow immediately from the construction here, but are not at all obvious for Schoen's solutions; we describe some of these below.

Let us describe the two approaches, and some of their differences, somewhat more specifically. If we normalize the desired constant scalar curvature $R$ to be $N(N-1)$, then the equation we are trying to solve on the sphere is

$$
\mathcal{N}(u) \equiv \Delta u-\frac{N(N-2)}{4} u+\frac{N(N-2)}{4} u^{\frac{N+2}{N-2}}=0 .
$$

In order to construct a solution with a given singular set $\Lambda$, first a one-parameter family of approximate solutions $\tilde{u}_{\varepsilon}$ of (4), each element of which blows up quickly enough near $\Lambda$, is constructed. Then (4) is linearized about $\tilde{u}_{\varepsilon}$, and the resulting linear operator $L_{\varepsilon}$ is analyzed. If it can be proved that $L_{\varepsilon}$ is surjective on some reasonable space of functions, at least when $\varepsilon$ is sufficiently small, then a standard iteration argument may be used to correct $\tilde{u}_{\varepsilon}$ by adding to it a function $v$ to obtain an exact solution to (1) which blows up sufficiently quickly at $\Lambda$. 
Unfortunately, $L_{\varepsilon}$ is not surjective on $L^{2}$; in fact, $L_{\varepsilon}$ is self-adjoint on $L^{2}$, but 0 is in its continuous spectrum, so that it does not even have closed range on this space. Schoen's tactic is to find an explicit infinite set of functions which span an approximate nullspace $K$, such that $L_{\varepsilon}$ is invertible on $K^{\perp}$. He first solves the equation on $K^{\perp}$, and then gives 'balancing' conditions to ensure that the solution of this restricted problem is a solution of the original problem. Our somewhat different tactic is to work on a finite dimensional extension of a certain weighted Hölder space. On this space, $L_{\varepsilon}$ is actually an isomorphism, when $\Lambda$ is discrete, and surjective in general, for $\varepsilon$ sufficiently small. Unfortunately, as $\varepsilon$ tends to zero, the norm of any right inverse for $L_{\varepsilon}$ blows up (Schoen encounters a similar problem). We must then analyze precisely the rate and manner of blowup. This is different than in [6], when $\Lambda^{\prime}$ is empty, and where the (right) inverse for $L_{\varepsilon}$ is bounded as $\varepsilon \rightarrow 0$. The advantage of working in weighted spaces over using the approximate nullspace $K$ is that in our approach there are only a finite number of obstructions to solving the equation, and these may be identified explicitly and geometrically. In fact, these obstructions are intimately connected with the definition of the finite dimensional extension mentioned above.

As noted earlier, another advantage of our approach is that we may easily derive various properties of the solutions. The main property we are interested in is nondegeneracy, which will be defined precisely in $\S 11$. This property is important in the study of the 'marked' and 'unmarked' moduli spaces $\mathcal{M}_{\Lambda}$ and $\mathcal{M}_{n}$ of solutions of this problem where the singular set $\Lambda$ is fixed or allowed to vary amongst all configurations of $n$ points in $S^{N}$. There are notions of marked and unmarked nondegeneracy associated to each of these spaces. These moduli spaces were defined and studied by the first author, with D. Pollack and K. Uhlenbeck in [7], [8]. It was proved that for $M=S^{N}$ they are real analytic sets. If there exists some $g \in \mathcal{M}_{\Lambda}$ which is marked nondegenerate, then the top dimensional stratum in the component of $g$ is a real analytic manifold of dimension $n$. Similarly, if $g \in \mathcal{M}_{n}$ is unmarked nondegenerate, then nearby $g$ this moduli space is a real analytic manifold of dimension $n(N+1)$. Nondegenerate solutions on $S^{N}$ of a very special type were constructed in [8]; for these solutions, only certain special configurations $\Lambda$ (in particular, only those with cardinality $n$ an even number and with points clustered in pairs) are allowed. In contrast, we prove

Theorem 2 For any integer $n \geq 2$ and any configuration $\Lambda$ of $n$ points in $S^{N}$ there exists an element $g \in \mathcal{M}_{n}$ which has singular set $\Lambda$ and which is unmarked nondegenerate. For a generic (in fact, Zariski open) set of $\Lambda$, this solution is marked nondegenerate, and for such a metric the points $\left(p_{1}, \ldots, p_{n}\right)$ of $\Lambda$ and the 'Delaunay necksizes' $\left(\varepsilon_{1}, \ldots, \varepsilon_{n}\right)$ constitute a full set of coordinates in $\mathcal{M}_{n}$ near $g$, while the Delaunay parameters alone yield coordinates in $\mathcal{M}_{\Lambda}$ near $g$.

The Delaunay necksizes will be defined in $\S 2$. We remark that the admissible sets of Delaunay necksize parameters $\left\{\varepsilon_{1}, \ldots, \varepsilon_{n}\right\}$ in the construction are not arbitrary sets of small numbers, but are required to satisfy a 'balancing conditions' (12).

Although the statement of Theorem 1 is for metrics on the complement in the sphere of a set $\Lambda$, we shall use the conformal equivariance of the equation (4) and prove instead the existence of solutions of this equation on the complement in $\mathbb{R}^{N}$ of a (finite) set $\Sigma$ which decay at infinity. This makes the technical details somewhat easier in that we always have a preferred coordinate system.

The solutions we construct here are not 'the same' as the ones constructed by R. Schoen; they are also quite different from the ones constructed in [8], and possibly don't even lie in the same components of the relevant moduli spaces. The ones in the latter paper are not required to have small Delaunay parameters, while (at least some of) the ones in [12] may be thought of as infinite strings of spheres, connected by very small necks, and joined together at a central convex sphere. Our solutions also have small necks, but the central region is metrically both concave and very small.

Karen Uhlenbeck has informed us that she too has established the existence of solutions to this problem with exactly one singular point on compact Riemannian manifolds $\left(M, g_{0}\right)$ with nonnegative conformal Laplacian. The construction of the approximate solutions in this case is 
substantially different than ours, and requires the positive mass theorem, but her linear analysis is in roughly the same spirit as ours.

The connection between this problem and the construction of complete, noncompact surfaces in $\mathbb{R}^{3}$ with constant mean curvature (CMC) is well-known. Indeed, Kapouleas' initial construction of these surfaces [3] is related to Schoen's construction of constant scalar curvature metrics [12], and the analysis of the moduli space problem in [4] for the CMC case was directly inspired by [7]. Maintaining this tradition, we shall show, in a work in preparation, the existence of complete, noncompact CMC surfaces in $\mathbb{R}^{3}$ with an arbitrary number of ends (greater than two), and with prescribed Delaunay parameters on these ends. These solutions are nondegenerate, hence are smooth points in the relevant moduli spaces. The geometric difference between these solutions and those of Kapouleas are the concavity in the central region of our solutions, versus the convexity of his.

The outline of this paper is as follows. In the first section we examine the basic models of singular solutions, the Delaunay solutions (the reason for this name is explained in [7]), and prove some estimates on them that will be required later. After this, we present the construction of approximate solutions for the problem. These functions are periodic near each end in a certain natural coordinate system, and correspondingly the linearized operators we must analyze have periodic coefficients. Although an analysis of such operators was made in [7], we need rather stronger behaviour than seems to be immediately available from the methods there, so the next few sections are devoted to a somewhat novel construction of inverses for the linearized operators. First of all, the linearized operators are invertible only on rather special function spaces. Then, we construct an inverse for the Dirichlet problems for the linearizations in the $\varepsilon$-neighbourhoods of the singular points, and on the complement of these neighbourhoods. By analyzing the Dirichletto-Neumann operators on the union of the boundaries of these neighbourhoods, we may join these right inverses together to get a global right inverse. To carry this out, we take a slightly indirect path and prove the existence of inverses by this method only for a model problem; the existence of inverses for the true problem is deduced by a rather intricate sequence of perturbations. After proving sufficiently fine estimates for these inverses, a rather standard contraction mapping argument (estimates for which, unfortunately, are not so trivial) is employed to complete the construction. After this we discuss the issue of nondegeneracy of the solutions. As noted earlier, in most of the paper we discuss only the case where $\Lambda$ is discrete, but in the last section we discuss the changes needed to handle the general case.

\section{Delaunay type solutions}

In this section we recall some well known fact about the Delaunay solutions of (1) on $S^{N}$ that will be used extensively in the rest of the paper. A reference for facts not proved here is [7], cf. also [13]. If $w$ is any solution of (1) on $S^{N} \backslash\left\{p_{1}, p_{2}\right\}$, then using the invariance with respect to any conformal transformation we may assume that these points are antipodal. Then, it is known that $u$ is rotationally invariant and thus the equation it satisfies may be reduced to an ODE. It is convenient to stereographically project the sphere $S^{N}$ to $\mathbb{R}^{N}$ from one of the singular points, say $p_{1}$, so that $p_{2}$ is sent to $0 \in \mathbb{R}^{N}$. Then the solution is transformed to a radial solution of

$$
\mathcal{N}(u) \equiv \Delta u+\frac{N(N-2)}{4} u^{\frac{N+2}{N-2}}=0,
$$

on $\mathbb{R}^{N} \backslash\{0\}$ which is singular at the origin.

We may reduce (5) by writing

$$
u(x)=|x|^{\frac{2-N}{2}} v(-\log |x|),
$$


and using $t=-\log |x|$ to get

$$
\frac{d^{2} v}{d t^{2}}-\frac{(N-2)^{2}}{4} v+\frac{N(N-2)}{4} v^{\frac{N+2}{N-2}}=0 .
$$

This equation is nondissipative, and the Hamiltonian energy

$$
H(v, \dot{v})=\dot{v}^{2}-\frac{(N-2)^{2}}{4} v^{2}+\frac{(N-2)^{2}}{4} v^{\frac{2 N}{N-2}}
$$

is constant along solutions of (6). Here and in all that follows, - denotes differentiation with respect to $t$. By examining the level curves of $H$, we see that all bounded solutions of (6) lie in the region of the $(v, \dot{v})$-plane where $H(v, \dot{v}) \leq 0$. There are several different types of bounded solutions; we summarize their basic properties:

Proposition 1 For any $H_{0} \in\left(-\frac{N-2}{2}\left(\frac{N-2}{N}\right)^{N / 2}, 0\right)$, there exists a unique bounded solution of $(6)$ satisfying $H(v, \dot{v})=H_{0}, \dot{v}(0)=0$ and $\ddot{v}(0)>0$. This solution is periodic and for all $t \in \mathbb{R}$ we have $v(t) \in(0,1)$. This solution can be indexed by the parameter $\varepsilon=v(0) \in\left(0,\left(\frac{N-2}{N}\right)^{(N-2) / 4}\right)$, which is the smaller of the two values $v$ assumes when $\dot{v}=0$. When $H_{0}=-\frac{N-2}{2}\left(\frac{N-2}{N}\right)^{N / 2}$, there is a unique bounded solution of (6), given by

$$
v(t)=\left(\frac{N-2}{N}\right)^{(N-2) / 4} .
$$

Finally, if $v$ is a solution with $H_{0}=0$ then either $v(t) \equiv 0$ or $v(t)=\left(\cosh \left(t-t_{0}\right)\right)^{\frac{2-N}{2}}$ for some $t_{0} \in \mathbb{R}$.

We will call these the Delaunay type solutions. Although we do not know them explicitly, the next two propositions give sufficient information about their behaviour as $\varepsilon$ tends to zero for our purposes.

Proposition 2 Fix $\varepsilon \in\left(0,\left(\frac{N-2}{N}\right)^{(N-2) / 4}\right)$ and let $v_{\varepsilon}$ be the corresponding Delaunay solution. Then the period $T_{\varepsilon}$ of $v_{\varepsilon}$ tends to infinity monotonically as $\varepsilon \rightarrow 0$ and satisfies

$$
T_{\varepsilon}=-\frac{4}{N-2} \log (\varepsilon)+O(1) .
$$

In addition, for all $t \in \mathbb{R}$

$$
\varepsilon \leq v_{\varepsilon}(t) \leq \varepsilon \cosh \left(\frac{N-2}{2} t\right)
$$

Proof : The second claim is rather simple. Since $H$ is constant along solutions,

$$
H\left(v_{\varepsilon}, \dot{v}_{\varepsilon}\right)=\dot{v}_{\varepsilon}^{2}-\frac{(N-2)^{2}}{4}\left(v_{\varepsilon}^{2}-v_{\varepsilon}^{\frac{2 N}{N-2}}\right)=-\frac{(N-2)^{2}}{4}\left(\varepsilon^{2}-\varepsilon^{\frac{2 N}{N-2}}\right) .
$$

Moreover, it is easy to see that $v_{\varepsilon}$ is increasing for $t \in\left[0, T_{\varepsilon} / 2\right]$ and decreasing for $t \in\left[T_{\varepsilon} / 2, T_{\varepsilon}\right]$. In particular the minimum of $v_{\varepsilon}$ is achieved at $t=0$ and equals $\varepsilon$. Using this lower bound in (8) we see that

$$
\dot{v}_{\varepsilon}^{2}=\frac{(N-2)^{2}}{4}\left(\left(v_{\varepsilon}^{2}-\varepsilon^{2}\right)-\left(v_{\varepsilon}^{\frac{2 N}{N-2}}-\varepsilon^{\frac{2 N}{N-2}}\right)\right) \leq \frac{(N-2)^{2}}{4}\left(v_{\varepsilon}^{2}-\varepsilon^{2}\right) .
$$

Taking the (positive) square root and integrating this differential inequality yields the inequality $v_{\varepsilon}(t) \leq \varepsilon \cosh \left(\frac{N-2}{2} t\right)$. 
Now we derive the asymptotic estimate for the period $T_{\varepsilon}$. Let $v_{\max , \varepsilon}$ denote the larger of the two solutions of

$$
v^{2}-v^{\frac{2 N}{N-2}}=\varepsilon^{2}-\varepsilon^{\frac{2 N}{N-2}} .
$$

(Hence $v_{\max , \varepsilon}$ is the larger of the two values $v_{\varepsilon}$ assumes when $\dot{v}_{\varepsilon}=0$ ). By (8), we get

$$
\int_{0}^{T_{\varepsilon} / 2}\left(v_{\varepsilon}^{2}-v_{\varepsilon}^{\frac{2 N}{N-2}}-\varepsilon^{2}+\varepsilon^{\frac{2 N}{N-2}}\right)^{-1 / 2} \dot{v}_{\varepsilon} d t=\frac{N-2}{4} T_{\varepsilon} .
$$

In the interval of integration we may change the variable of integration to $\bar{v}=v_{\varepsilon}(t) / \varepsilon$. This yields

$$
\int_{\varepsilon}^{v_{\max , \varepsilon}}\left(\bar{v}^{2}-\varepsilon^{2}-\left(\bar{v}^{\frac{2 N}{N-2}}-\varepsilon^{\frac{2 N}{N-2}}\right)\right)^{-1 / 2} d \bar{v}=\frac{N-2}{4} T_{\varepsilon} .
$$

It is then not difficult to see that this integral grows as $-\log \varepsilon+O(1)$. A proof of the fact that the period is a monotone function of $\varepsilon$ is indicated in [7] and also proved by elementary ODE methods in an unpublished manuscript of Chouikha and Weissler.

Proposition 3 For any $\varepsilon \in\left(0,\left(\frac{N-2}{N}\right)^{(N-2) / 4}\right)$ and for any $t \in \mathbb{R}$ the Delaunay solution $v_{\varepsilon}$ satisfies the estimates

$$
\begin{gathered}
\left|v_{\varepsilon}(t)-\varepsilon \cosh \left(\frac{N-2}{2} t\right)\right| \leq c_{N} \varepsilon^{\frac{N+2}{N-2}} e^{\frac{N+2}{2}|t|}, \\
\left|\dot{v}_{\varepsilon}(t)-\frac{N-2}{2} \varepsilon \sinh \left(\frac{N-2}{2} t\right)\right| \leq c_{N} \varepsilon^{\frac{N+2}{N-2}} e^{\frac{N+2}{2}|t|},
\end{gathered}
$$

for some constant $c_{N}>0$ which depends only on $N$.

Proof : We start with the a priori estimate

$$
v_{\varepsilon}(t) \leq \varepsilon \cosh \left(\frac{N-2}{2} t\right) \leq \varepsilon e^{\frac{N-2}{2} t}
$$

valid for $t>0$. Next, writing the equation for $v_{\varepsilon}$ as

$$
\frac{d^{2} v}{d t^{2}}-\frac{(N-2)^{2}}{4} v=-\frac{N(N-2)}{4} v^{\frac{N+2}{N-2}}
$$

we can represent $v_{\varepsilon}$ as

$$
v_{\varepsilon}(t)=\varepsilon \cosh \left(\frac{N-2}{2} t\right)-\frac{N(N-2)}{4} e^{\frac{N-2}{2} t} \int_{0}^{t} e^{(2-N) s} \int_{0}^{s} e^{\frac{N-2}{2} z} v_{\varepsilon}^{\frac{N+2}{N-2}}(z) d z d s .
$$

Together with the a priori estimates above, this leads immediately to

$$
0 \leq \varepsilon \cosh \left(\frac{N-2}{2} t\right)-v_{\varepsilon}(t) \leq \frac{N-2}{8} \varepsilon^{\frac{N+2}{N-2}} e^{\frac{N+2}{2} t} .
$$

Finally, differentiating the formula of $v_{\varepsilon}$ with respect to $t$, we get

$$
\begin{gathered}
\left|\dot{v}_{\varepsilon}(t)-\frac{N-2}{2} \varepsilon \sinh \left(\frac{N-2}{2} t\right)\right| \leq \frac{N(N-2)}{4}\left(\frac{N-2}{2} e^{\frac{N-2}{2} t} \int_{0}^{t} e^{(2-N) s} \int_{0}^{s} e^{\frac{N-2}{2} z} v_{\varepsilon}^{\frac{N+2}{N-2}}(z) d z d s\right. \\
\left.+e^{\frac{2-N}{2} t} \int_{0}^{t} e^{\frac{N-2}{2} z} v^{\frac{N+2}{N-2}}(z) d z d s\right) \leq \frac{N^{2}-4}{16} \varepsilon^{\frac{N+2}{N-2}} e^{\frac{N+2}{2} t} .
\end{gathered}
$$


Remark 1 The estimate of this Lemma is only interesting in the domain where

$$
2 c_{N} \varepsilon^{\frac{N+2}{N-2}} e^{\frac{N+2}{2}|t|}<(N-2) \varepsilon e^{\frac{N-2}{2}|t|},
$$

i.e. where $|t|<-\frac{2}{N-2} \log \varepsilon-\tilde{c}_{N}$, which is close to half a period of $v_{\varepsilon}$.

We transfer these estimates and this remark back to the $x$-coordinates to obtain

Corollary 1 For any $\varepsilon \in\left(0,\left(\frac{N-2}{N}\right)^{(N-2) / 4}\right)$ and any $x \in \mathbb{R}^{N} \backslash\{0\}$, the Delaunay solution $u_{\varepsilon}(x)$ satisfies the estimates

$$
\begin{gathered}
\left|u_{\varepsilon}(x)-\frac{\varepsilon}{2}\left(1+|x|^{2-N}\right)\right| \leq C_{N} \varepsilon^{\frac{N+2}{N-2}}|x|^{-N}, \\
\left.\left.\left|r \partial_{r} u_{\varepsilon}(x)+\frac{N-2}{2} \varepsilon\right| x\right|^{2-N}\left|\leq C_{N} \varepsilon^{\frac{N+2}{N-2}}\right| x\right|^{-N} .
\end{gathered}
$$

In particular, in the annulus $\varepsilon^{\frac{2}{N-2}} \leq|x| \leq 1, u_{\varepsilon}$ is well-approximated by $\varepsilon|x|^{2-N} / 2$.

There are some important variations of these solutions, leading to a $2 N+2$-dimensional family of Delaunay type solutions. These variations are families of solutions $U(s)$ of $\mathcal{N}(U(s))=0$ with $U(0)=u_{\varepsilon}$, depending smoothly on the parameter $s$. The derivatives of these families with respect to $s$ at $s=0$ correspond to Jacobi fields, i.e. solutions of the linearization of $\mathcal{N}$ about one of the $u_{\varepsilon}$, and will be described below.

We describe these families of variations in turn. The first is quite obvious. It is the family where the Delaunay parameter $\varepsilon$ is varied

$$
(-\varepsilon, 1-\varepsilon) \ni \eta \longrightarrow u_{\varepsilon+\eta}(x) .
$$

The second corresponds to the fact that if $u$ is any solutions of $\mathcal{N}(u)=0$, then $R^{\frac{2-N}{2}} u(x / R)$ also solves this equation. Applying this to a Delaunay solution yields the family

$$
\mathbb{R}^{+} \ni R \longrightarrow|x|^{\frac{2-N}{2}} v_{\varepsilon}(-\log |x|+\log R) .
$$

The other two families of solutions correspond to translations. The simpler of these is the usual translation

$$
\mathbb{R}^{N} \ni b \rightarrow u_{\varepsilon}(x+b) .
$$

The final one corresponds to translations 'at infinity'. To describe this we use the Kelvin transform, $u(x) \rightarrow|x|^{2-N} u\left(\frac{x}{|x|^{2}}\right)$, which preserves the property of being a weak solution of (5). Start with a Delaunay solution $u_{\varepsilon}(x)$. Its Kelvin transform is

$$
|x|^{\frac{2-N}{2}} v_{\varepsilon}(\log |x|) .
$$

Translate this by some $a \in \mathbb{R}^{N}$ to get

$$
|x-a|^{\frac{2-N}{2}} v_{\varepsilon}(\log |x-a|),
$$

which has its singularity at $a$ rather than 0 . Its Kelvin transform yields the family

$$
\left.\left.\mathbb{R}^{N} \ni a \rightarrow|x-a| x\right|^{2}\right|^{\frac{2-N}{2}} v_{\varepsilon}\left(-2 \log |x|+\left.\log |x-a| x\right|^{2} \mid\right) .
$$

Each function in this family has singularity at 0 again.

In fact, the first and third variations, where the Delaunay parameter is changed or where the singularity is moved, are less well behaved than the other two: the Jacobi fields corresponding to them grow too quickly. Thus we shall focus attention on the smaller family of solutions

$$
u_{\varepsilon}(R, a, x)=\left.\left.|x-a| x\right|^{2}\right|^{\frac{2-N}{2}} v_{\varepsilon}\left(-2 \log |x|+\left.\log |x-a| x\right|^{2} \mid+\log R\right),
$$

where only translations along the Delaunay axis and of the 'point at infinity' are allowed. 


\section{Construction of the approximate solutions}

We now proceed to define a family of approximate solutions to the problem, using the Delaunay solutions of the last section. Although we ultimately wish to construct solutions on $S^{N} \backslash \Lambda$, we shall use the conformal invariance of the problem and construct solutions on $\mathbb{R}^{N} \backslash \Sigma$, where

$$
\Sigma=\left\{x_{1}, \ldots, x_{n}\right\}
$$

with appropriate regularity at infinity. This is done purely to make the notation as simple as possible.

One can always assume that all the $B\left(x_{i}, 2\right)$ are disjoint since we may dilate our problem by some factor $\kappa>0$ which will change the set $\Sigma$ into $\kappa \Sigma$ and the function $u$ defined in $\mathbb{R}^{N} \backslash \Sigma$ into $\kappa^{\frac{2-N}{2}} u(\cdot / \kappa)$ defined in $\mathbb{R}^{N} \backslash \kappa \Sigma$.

The approximate solutions we define here have Delaunay type singularities in a ball $B\left(x_{i}, \rho_{i}\right)$ around each point of $\Sigma$ and are harmonic outside the union of slightly larger balls. We use not just the radial Delaunay solutions, but elements of the family $u_{\varepsilon}(R, a, x)$ defined above. The additional parameters in this family yield, on the linear level, the Jacobi fields constituting a 'deficiency space' needed to obtain surjectivity of the linearized operator. In addition, these extra parameters are essential in ensuring a sufficiently good agreement in the transition regions around the boundaries of the small balls. We emphasize again that we do not use all possible Delaunay solutions in each ball, but just two of the four possible families.

Let

$$
\bar{\varepsilon}=\left(\varepsilon_{1}, \ldots, \varepsilon_{n}\right), \quad \bar{R}=\left(R_{1}, \ldots, R_{n}\right), \quad \bar{\rho}=\left(\rho_{1}, \ldots, \rho_{n}\right), \quad \text { and } \quad \bar{a}=\left(a_{1}, \ldots, a_{n}\right)
$$

be sets of (small) Delaunay parameters, positive real numbers corresponding to translations of the Delaunay solutions, (small) radii and vectors in $\mathbb{R}^{N}$, respectively. We shall impose various constraints on these quantities. First, assume that the balls $B\left(x_{i}, \rho_{i}\right)$ do not intersect one another. We will denote by $\Omega_{\bar{\rho}}$ the set $\mathbb{R}^{N} \backslash \cup_{i=1}^{n} B\left(x_{i}, \rho_{i}\right)$. Next, we shall assume that

$$
\rho_{i}=\varepsilon_{i}^{\frac{4}{N^{2}-4}} .
$$

Finally, fix a set of positive numbers $q_{1}, \ldots, q_{n}$, and assume that

$$
\varepsilon_{i}=\varepsilon q_{i}, \quad i=1, \ldots, n,
$$

or simply $\bar{\varepsilon}=\varepsilon \bar{q}$, for some $\varepsilon>0$. We shall call a set $\bar{\varepsilon}$ satisfying (11) admissible. Notice that (10) and (11) imply that the quantities $\varepsilon_{1}, \ldots, \varepsilon_{n}$ are all comparable to one another, and similarly for the $\rho_{i}$. Because of this, it makes sense to estimate various quantities later by $\varepsilon$ or $\rho=\varepsilon^{4 /\left(N^{2}-4\right)}$.

We shall now define the family of approximate solutions $\bar{u}_{\bar{\varepsilon}}(\bar{R}, \bar{a}, \cdot)$. In each ball $B\left(x_{i}, \rho_{i}\right)$, $\bar{u}_{\bar{\varepsilon}}(\bar{R}, \bar{a}, x)$ is equal to $u_{\varepsilon_{i}}\left(R_{i}, a_{i}, x-x_{i}\right)$. On the exterior region $\Omega_{\bar{\rho}}, \bar{u}_{\bar{\varepsilon}}(\bar{R}, \bar{a}, x)$ will equal a harmonic function $w(x)$. With only this restriction, it is possible to make $\bar{u}_{\bar{\varepsilon}}(\bar{R}, \bar{a}, x)$ continuous across each $\partial B\left(x_{i}, \rho_{i}\right)$, but not $\mathcal{C}^{1}$. For example, if one takes $w(x)$ to be the unique harmonic function decaying at infinity with values on $\partial B\left(x_{i}, \rho_{i}\right)$ given by $u_{\varepsilon_{i}}\left(R_{i}, a_{i}, x-x_{i}\right)$, then by definition $\bar{u}_{\bar{\varepsilon}}(\bar{R}, \bar{a}, x)$ is continuous but not $\mathcal{C}^{1}$. However, we do not know this harmonic function all that explicitly, and so it is much easier to deal instead with a harmonic function which is a sum of multiples of Green functions with poles at the $x_{i}$. With such a choice we can no longer make the approximate solution even $\mathcal{C}^{0}$, but the next result shows that we can mitigate this discontinuity to an extent needed for the later argument, by choosing all parameters carefully.

Proposition 4 Suppose that $\bar{\varepsilon}$ is a set of admissible Delaunay parameters, with $\varepsilon<\varepsilon_{0}$. Suppose that the parameters $R_{i}$ and $a_{i}$ satisfy the relationships

$$
\sum_{i \neq i_{0}} R_{i}^{\frac{N-2}{2}} R_{i_{0}}^{\frac{N-2}{2}} q_{i}\left|x_{i_{0}}-x_{i}\right|^{2-N}=q_{i_{0}}, \quad i_{0}=1, \ldots, n
$$


and

$$
a_{i_{0}}=-\frac{1}{q_{i_{0}}} R_{i_{0}}^{\frac{N-2}{2}} \sum_{i \neq i_{0}} q_{i} R_{i}^{\frac{N-2}{2}}\left|x_{i_{0}}-x_{i}\right|^{-N}\left(x_{i_{0}}-x_{i}\right),
$$

and that the harmonic function $\bar{w}_{\bar{\varepsilon}}(\bar{R}, x)$ on $\Omega_{\bar{\rho}}$ is given by

$$
\bar{w}_{\bar{\varepsilon}}(\bar{R}, x)=\sum_{i=1}^{n} \frac{\varepsilon_{i}}{2} R_{i}^{\frac{N-2}{2}}\left|x-x_{i}\right|^{2-N} .
$$

Then, for $i=1, \ldots, n$ and $j=0, \ldots, N$, we have the estimates

$$
\int_{S^{N-1}}\left(u_{\varepsilon_{i}}\left(R_{i}, a_{i}, \cdot-x_{i}\right)-\bar{w}_{\bar{\varepsilon}}(\bar{R}, \cdot)\right)\left(x_{i}+\rho_{i} \theta\right) \phi_{j}(\theta) d \theta=O\left(\varepsilon \rho^{2}\right),
$$

and

$$
\int_{S^{N-1}}\left(\frac{\partial u_{\varepsilon_{i}}\left(R_{i}, a_{i}, \cdot-x_{i}\right)}{\partial r_{i}}-\frac{\partial \bar{w}_{\bar{\varepsilon}}(\bar{R}, \cdot)}{\partial r_{i}}\right)\left(x_{i}+\rho_{i} \theta\right) \phi_{j}(\theta) d \theta=O(\varepsilon \rho),
$$

Here $\phi_{j}(\theta)=\theta_{j}, j=0, \ldots, N$, are eigenfunctions corresponding to the first two eigenvalues of the Laplacian on $S^{N-1}$, i.e. $\lambda_{0}=0$ and $\lambda_{1}=N-1$.

Remark 2 The restrictions imposed on the parameters by this proposition may be regarded as global balancing conditions for the approximate solutions. However, they are rather different in their technical use from the balancing conditions used by Schoen in [12]. In fact, our conditions simply ensure that there is a closest possible degree of contact between an approximate solution inside each of the balls $B\left(x_{i}, \rho_{i}\right)$, where it is equal to a deformed Delaunay solution, and outside the union of these balls, where it is a harmonic function.

Remark 3 It is not immediately clear that there are choices of parameters satisfying both (12) and (13), but we can see that such choices do exist by the following argument. Equation (12) says that $\bar{q}$ is an eigenvector with eigenvalue 1 of some matrix, the coefficients of which depend on the $R_{i}$ and $x_{i}$. Since we are fixing the $x_{i}$, and are considering $\bar{q}$ as fixed, we then regard (12) as an equation for the $R_{i}$. This equation may not have solutions for every $\bar{q}$, but it is not hard to show that there is a cone in the positive orthant of the $\bar{q}$ space for which solutions of this equation do exist. Once we have chosen the $R_{i}$ of course, then we can use (13) to determine the $a_{i}$. One further comment on these quantities. Many of the constants in estimates throughout this paper depend in some manner on these parameters. Since we are considering $\bar{R}$ and $\bar{a}$ fixed, we often neglect to mention the dependence of various constants on them.

Proof: To prove these estimates, we shall expand both $u_{\varepsilon_{i}}\left(R_{i}, a_{i}, x-x_{i}\right)$ and $\bar{w}_{\bar{\varepsilon}}(\bar{R}, x)$ near $\partial B\left(x_{i}, \rho_{i}\right)$. First we consider $u_{\varepsilon_{i}}\left(R_{i}, a_{i}, x-x_{i}\right)$. From Proposition 3 , if $|a| \rho \leq 1 / 8$ as we may well assume by taking $\rho$ small, then near $\partial B\left(x_{i}, \rho_{i}\right)$ we have

$$
u_{\varepsilon_{i}}\left(R_{i}, a_{i}, x-x_{i}\right)=\frac{\varepsilon_{i}}{2}\left(R_{i}^{\frac{N-2}{2}}\left|x-x_{i}\right|^{2-N}+R_{i}^{\frac{2-N}{2}}\left(1+(N-2)\left(a_{i} \cdot\left(x-x_{i}\right)\right)\right)+O\left(\varepsilon \rho^{2}\right) .\right.
$$

It is precisely at this step, in estimating the size of the error term, that we are led to fix the size of the balls using $(10)$, since with this definition of $\rho$ we have $\varepsilon \rho^{2}=\varepsilon^{\frac{N+2}{N-2}} \rho^{-N}$. We also have

$$
\partial_{r_{i}} u_{\varepsilon_{i}}\left(R_{i}, a_{i}, x-x_{i}\right)=-\frac{N-2}{2} \varepsilon_{i}\left(R_{i}^{\frac{N-2}{2}}\left|x-x_{i}\right|^{1-N}-R_{i}^{\frac{2-N}{2}}\left|x-x_{i}\right|^{-1}\left(a_{i} \cdot\left(x-x_{i}\right)\right)\right)+O(\varepsilon \rho) .
$$

Next, for the expansion of $\bar{w}_{\bar{\varepsilon}}(\bar{R}, x)$ near $\partial B\left(x_{i_{0}}, \rho_{i_{0}}\right)$ we have

$$
\begin{aligned}
\bar{w}_{\bar{\varepsilon}}(\bar{R}, x)= & \frac{\varepsilon_{i_{0}}}{2} R_{i_{0}}^{\frac{N-2}{2}}\left|x-x_{i_{0}}\right|^{2-N}+\sum_{i \neq i_{0}} \frac{\varepsilon_{i}}{2} R_{i}^{\frac{N-2}{2}}\left(\left|x_{i_{0}}-x_{i}\right|^{2-N}\right. \\
& \left.+(2-N)\left|x_{i_{0}}-x_{i}\right|^{-N}\left(x_{i_{0}}-x_{i}\right) \cdot\left(x-x_{i_{0}}\right)\right)+O\left(\varepsilon \rho^{2}\right),
\end{aligned}
$$


and

$$
\begin{aligned}
\partial_{r_{i_{0}}} \bar{w}_{\bar{\varepsilon}}(\bar{R}, x)= & (2-N)\left(\frac{\varepsilon_{i_{0}}}{2} R_{i_{0}}^{\frac{N-2}{2}}\left|x-x_{i_{0}}\right|^{1-N}\right. \\
& \left.+\sum_{i \neq i_{0}} \frac{\varepsilon_{i}}{2} R_{i}^{\frac{N-2}{2}}\left|x_{i_{0}}-x_{i}\right|^{-N}\left|x-x_{i_{0}}\right|^{-1}\left(x_{i_{0}}-x_{i}\right) \cdot\left(x-x_{i_{0}}\right)\right)+O(\varepsilon \rho) .
\end{aligned}
$$

Taking the difference of these expressions we obtain, near $\left|x-x_{i_{0}}\right|=\rho_{i_{0}}$, that

$$
\begin{gathered}
u_{\varepsilon_{i_{0}}}\left(R_{i_{0}}, a_{i_{0}}, x-x_{i_{0}}\right)-\bar{w}_{\bar{\varepsilon}}(\bar{R}, x)=\left(\frac{\varepsilon_{i_{0}}}{2} R_{i_{0}}^{\frac{2-N}{2}}-\sum_{i \neq i_{0}} \frac{\varepsilon_{i}}{2} R_{i}^{\frac{N-2}{2}}\left|x_{i_{0}}-x_{i}\right|^{2-N}\right) \\
+(N-2)\left(\frac{\varepsilon_{i_{0}}}{2} R_{i_{0}}^{\frac{2-N}{2}} a_{i_{0}}+\sum_{i \neq i_{0}} \frac{\varepsilon_{i}}{2} R_{i}^{\frac{N-2}{2}}\left|x_{i_{0}}-x_{i}\right|^{-N}\left(x_{i_{0}}-x_{i}\right)\right) \cdot\left(x-x_{i_{0}}\right)+O\left(\varepsilon \rho^{2}\right)
\end{gathered}
$$

and

$$
\begin{gathered}
\partial_{r_{i_{0}}}\left(u_{\varepsilon_{i_{0}}}\left(R_{i_{0}}, a_{i_{0}}, x-x_{i_{0}}\right)-\bar{w}_{\bar{\varepsilon}}(\bar{R}, x)\right)=(N-2)\left(\frac{\varepsilon_{i_{0}}}{2} R_{i_{0}}^{\frac{2-N}{2}} a_{i_{0}}+\right. \\
\left.\sum_{i \neq i_{0}} \frac{\varepsilon_{i}}{2} R_{i}^{\frac{N-2}{2}}\left|x_{i_{0}}-x_{i}\right|^{-N}\left(x_{i_{0}}-x_{i}\right)\right) \cdot \frac{x-x_{i_{0}}}{\left|x-x_{i_{0}}\right|}+O(\varepsilon \rho) .
\end{gathered}
$$

Now insert (12) and (13) into these expressions, using (10) again, to obtain the result.

Notice, and this will be useful, that the expansions in the proof above hold for $\rho_{i} \leq\left|x_{x}-i\right| \leq 2$. Finally, using the function $\bar{w}_{\bar{\varepsilon}}(\bar{R}, x)$ given in Proposition 4, we define the approximate solution

$$
\bar{u}_{\bar{\varepsilon}}(\bar{R}, \bar{a}, x)=\sum_{i=1}^{n} \chi_{i}\left(x-x_{i}\right) u_{\varepsilon_{i}}\left(R_{i}, a_{i}, x-x_{i}\right)+\bar{w}_{\bar{\varepsilon}}(\bar{R}, x)\left(1-\sum_{i=1}^{n} \chi_{i}\left(x-x_{i}\right)\right) .
$$

Here the $\chi_{i}$ are smooth, radial functions equalling one in $|x| \leq \rho_{i}$, vanishing in $|x| \geq 2 \rho_{i}$, and satisfy $\left|\partial_{r} \chi_{i}(x)\right| \leq c \rho_{i}^{-1}$ and $\left|\partial_{r}^{2} \chi_{i}(x)\right| \leq c \rho_{i}^{-2}$. Notice that we may define the approximate solution $\bar{u}_{\bar{\varepsilon}}(\bar{R}, \bar{a}, x)$ regardless of whether the relations (12) and (13) are satisfied.

We conclude this section by stating an estimate which will be used extensively later, and which follows easily from the proof of Proposition 4.

Corollary 2 Let $c>0$ be given and suppose that $\bar{R}$ satisfies (12) and that $|\bar{a}| \leq c$. Then for $\rho_{i} \leq\left|x-x_{i}\right| \leq 1$

$$
\left|\bar{u}_{\bar{\varepsilon}}(\bar{R}, \bar{a}, x)^{\frac{4}{N-2}}-u_{\varepsilon_{i}}\left(R_{i}, a_{i}, x-x_{i}\right)^{\frac{4}{N-2}}\right| \leq C \varepsilon^{\frac{4}{N-2}}\left|x-x_{i}\right|^{N-5}
$$

and

$$
\left|\bar{u}_{\bar{\varepsilon}}(\bar{R}, \bar{a}, x)^{\frac{4}{N-2}}-\bar{u}_{\bar{\varepsilon}}(\bar{R}, 0, x)^{\frac{4}{N-2}}\right| \leq C \varepsilon^{\frac{4}{N-2}}\left|x-x_{i}\right|^{N-5} .
$$

Proof: First of all, notice that the expansions of the proof of the previous Proposition do hold for all $\rho_{i} \leq\left|x-x_{i}\right| \leq 1$. To obtain the first estimate, we write $\bar{u}_{\bar{\varepsilon}}$ and $u_{\varepsilon_{i}}$ for the functions appearing in this estimate. Also, assume $x_{i}=0$ for convenience. We write the quantity to be estimated as

$$
\left|\left(u_{\varepsilon_{i}}+\left(1-\chi_{i}\right)\left(\bar{w}_{\bar{\varepsilon}}-u_{\varepsilon_{i}}\right)\right)^{\frac{4}{N-2}}-u_{\varepsilon_{i}}^{\frac{4}{N-2}}\right| .
$$


By Taylor's theorem, this is dominated by

$$
C\left(u_{\varepsilon_{i}}\right)^{\frac{4}{N-2}-1}\left|u_{\varepsilon_{i}}-\bar{w}_{\bar{\varepsilon}}\right| .
$$

From (15), we see that when $\bar{R}$ solves (12), then $\left|u_{\varepsilon_{i}}-\bar{w}_{\bar{\varepsilon}}\right|$ is bounded by $C \varepsilon\left|x-x_{i}\right|$. Now use that $1 / C \varepsilon\left|x-x_{i}\right|^{2-N} \leq u_{\varepsilon_{i}} \leq C \varepsilon\left|x-x_{i}\right|^{2-N}$ to complete the proof of the first estimate.

To obtain the second estimate, we write the quantity to be estimated as

$$
\left|\left(\bar{w}_{\bar{\varepsilon}}+\chi_{i}\left(u_{\varepsilon_{i}}\left(R_{i}, a_{i}, \cdot\right)-\bar{w}_{\bar{\varepsilon}}\right)\right)^{\frac{4}{N-2}}-\bar{w}_{\bar{\varepsilon}}+\chi_{i}\left(u_{\varepsilon_{i}}\left(R_{i}, 0, \cdot\right)-\bar{w}_{\bar{\varepsilon}}\right)^{\frac{4}{N-2}}\right|
$$

which, by Taylor's theorem, is dominated by

$$
C\left(\bar{w}_{\bar{\varepsilon}}\right)^{\frac{4}{N-2}-1}\left|u_{\varepsilon_{i}}-\bar{w}_{\bar{\varepsilon}}\right| .
$$

The estimate follows at once from the fact that $1 / C \varepsilon\left|x-x_{i}\right|^{2-N} \leq \bar{w}_{\bar{\varepsilon}} \leq C \varepsilon\left|x-x_{i}\right|^{2-N}$.

\section{The linearized operator on $\mathbb{R}^{N} \backslash\{0\}$}

Much of the analysis in this paper concerns the linearization of the nonlinear operator $\mathcal{N}$ about one of the approximate solutions $\bar{u}_{\bar{\varepsilon}}(\bar{R}, \bar{a}, x)$. We shall approach the study of this linearization gradually. In this section we define the linearization about one of the Delaunay solutions $u_{\varepsilon}(R, a, x)$ on $\mathbb{R}^{N} \backslash\{0\}$. The main point here is the introduction of the Jacobi fields $\Psi_{\varepsilon, R, a}^{j, \pm}(x)$. In succeeding sections we discuss the refined mapping properties of the linearization, first for the Dirichlet problem on the unit ball, then for a simpler, locally radial model for the true linearization on $\mathbb{R}^{N} \backslash \Sigma$, and finally for the true linearization itself.

Fix one of the Delaunay solutions $u_{\varepsilon, R, a}$. The linearization of $\mathcal{N}$ at $u_{\varepsilon, R, a}$ is defined by

$$
L_{\varepsilon, R, a} v=\left.\frac{d}{d s}\right|_{s=0} \mathcal{N}\left(u_{\varepsilon}(R, a, \cdot)+s v\right)=\Delta v+\frac{N(N+2)}{4} u_{\varepsilon}(R, a, \cdot)^{\frac{4}{N-2}} v .
$$

More generally, this operator can also be defined as the derivative at $s=0$ of $\mathcal{N}(U(s))$, where $U(s)$ is any one-parameter family of solutions with $U(0)=u_{\varepsilon}(R, a, x), U^{\prime}(0)=v$. Viewed this way, it is immediate that varying the parameters in any one of the families of Delaunay solutions leads to solutions of $L_{\varepsilon, R, a} \Psi=0$. Solutions of this homogeneous problem are called Jacobi fields.

The Jacobi fields corresponding to these various families are easy to determine rather explicitly, cf. [7]. We shall be primarily interested, at least initially, in the Jacobi fields at $a=0$. Since the functions $u_{\varepsilon}(R, 0, x)$ are radial, we may separate variables in this case. Introduce the eigenfunctions $\phi_{j}(\theta)$ and eigenvalues $\lambda_{j}$ for the Laplacian on $S^{N-1}$. Then corresponding to $\lambda_{0}=0$ are the Jacobi fields for the first and second types of variations:

$$
\begin{aligned}
\Psi_{\varepsilon}^{0,-}(x) & =\partial_{\varepsilon} u_{\varepsilon}(1,0, x)=|x|^{\frac{2-N}{2}} \frac{\partial v_{\varepsilon}}{\partial \varepsilon}(-\log |x|), \\
& \equiv|x|^{\frac{2-N}{2}} \Phi_{\varepsilon}^{0,-}(-\log |x|) \\
\text { - } \Psi_{\varepsilon}^{0,+}(x) & =\partial_{R} u_{\varepsilon}(1,0, x)=-|x| \frac{\partial u_{\varepsilon}}{\partial r}(x)-\frac{N-2}{2} u_{\varepsilon}(x)=|x|^{\frac{2-N}{2}} \frac{\partial v_{\varepsilon}}{\partial t}(-\log |x|), \\
& \equiv|x|^{\frac{2-N}{2}} \Phi_{\varepsilon}^{0,+}(-\log |x|)
\end{aligned}
$$

Here we take $\phi_{0}(\theta)=1$. The third and fourth families have Jacobi fields corresponding to the next set of eigenvalues, $\lambda_{1}=\ldots=\lambda_{N}=N-1$. These are:

$$
\begin{aligned}
\Psi_{\varepsilon}^{j,+}(x) & =\partial_{a_{j}} u_{\varepsilon}(1,0, x)=|x|^{\frac{4-N}{2}}\left(\frac{N-2}{2} v_{\varepsilon}(-\log |x|)-\frac{\partial v_{\varepsilon}}{\partial t}(-\log |x|)\right) \phi_{j}(\theta), \\
& \equiv|x|^{\frac{2-N}{2}} \Phi_{\varepsilon}^{1,+}(-\log |x|) \phi_{j}(\theta)
\end{aligned}
$$




$$
\begin{aligned}
\Psi_{\varepsilon}^{j,-}(x) & =\partial_{x_{j}} u_{\varepsilon}(1,0, x)=|x|^{-\frac{N}{2}}\left(\frac{2-N}{2} v_{\varepsilon}(-\log |x|)-\frac{\partial v_{\varepsilon}}{\partial t}(-\log |x|)\right) \phi_{j}(\theta) \\
& \equiv|x|^{\frac{2-N}{2}} \Phi_{\varepsilon}^{1,-}(-\log |x|) \phi_{j}(\theta)
\end{aligned}
$$

Later we shall also use the Jacobi fields corresponding to differentiating the family $u_{\varepsilon}(R, a, x)$ and evaluating not necessarily at $R=1, a=0$. We shall denote these Jacobi fields by

$$
\Psi_{\varepsilon, R, a}^{j, \pm}(x) \equiv|x|^{\frac{2-N}{2}} \Phi_{\varepsilon, R, a}^{j, \pm}(x), \quad j=0, \cdots, N .
$$

Finally, when $a \equiv 0$ we shall denote these Jacobi fields by

$$
\Psi_{\varepsilon, R}^{j, \pm}(x) \equiv|x|^{\frac{2-N}{2}} \Phi_{\varepsilon, R}^{j, \pm}(x), \quad j=0, \cdots, N .
$$

\section{$5 \quad$ Function spaces}

We shall consider the action of the linearized operator on weighted Hölder spaces, which we now define. Since we shall be considering both local and global versions of this linearization, we first define the appropriate spaces on the ball:

Definition 1 For each $k \in \mathbb{N}, 0<\alpha<1$ and $\sigma \in \mathbb{R}^{+}$set

$$
|u|_{k, \alpha,[\sigma, 2 \sigma]}=\sup _{|x| \in[\sigma, 2 \sigma]}\left(\sum_{j=0}^{k} \sigma^{j}\left|\nabla^{j} u(x)\right|\right)+\sigma^{k+\alpha} \sup _{|x|,|y| \in[\sigma, 2 \sigma]}\left(\frac{\left|\nabla^{k} u(x)-\nabla^{k} u(y)\right|}{|x-y|^{\alpha}}\right) .
$$

Then, for any $\mu \in \mathbb{R}$, the space $\mathcal{C}_{\mu}^{k, \alpha}(B(0, R) \backslash\{0\})$ is the collection of functions $u$ which are locally in $\mathcal{C}^{k, \alpha}(B(0, R) \backslash\{0\})$ and for which the norm

$$
\|u\|_{k, \alpha, \mu}=\sup _{0<\sigma \leq R / 2} \sigma^{-\mu}|u|_{k, \alpha,[\sigma, 2 \sigma]}
$$

is finite. The subspace $\mathcal{C}_{\mu, \mathcal{D}}^{k, \alpha}(B(0, R) \backslash\{0\})$ consists of those functions which, in addition, vanish at the boundary $|x|=R$.

In particular, the function $r^{\mu}$ is in $\mathcal{C}_{\mu}^{k, \alpha}$ for any $k, \alpha$ or $\mu$.

There are analogous spaces of functions on the complement of the prescribed singular set in $S^{N}$ of the eventual solution defined in the obvious way. Since it is easier to work in Euclidean coordinates, we shall usually consider instead the induced operator and problem on $\mathbb{R}^{N} \backslash \Sigma$, where $\Sigma=\left\{x_{1}, \ldots, x_{n}\right\}$. The only difference is that we now need to consider decay properties near Euclidean infinity. Consideration of these is included in the following definition. Divide $\mathbb{R}^{N} \backslash \Sigma$ into the union of three slightly overlapping open regions: $\Omega_{1} \equiv \cup_{i=1}^{n} B\left(x_{i}, 1\right)$, a neighbourhood of $\Sigma, \Omega_{2} \equiv\{|x|>1 / C\}$, a neighbourhood of infinity, and a bounded piece $\Omega_{3}$.

Definition 2 For any $\nu, \nu^{\prime} \in \mathbb{R}$, the space $\mathcal{C}_{\nu, \nu^{\prime}}^{k, \alpha}\left(\mathbb{R}^{N} \backslash \Sigma\right)$ is defined to be the collection of $u \in$ $\mathcal{C}^{k, \alpha}\left(\mathbb{R}^{N} \backslash \Sigma\right)$ for which the norm

$$
\|u\|_{k, \alpha, \nu, \nu^{\prime}}=\|u\|_{k, \alpha, \nu, \Omega_{1}}+\left\||x|^{-N+\nu^{\prime}} u\left(x /|x|^{2}\right)\right\|_{k, \alpha, B(0, C)}+\|u\|_{k, \alpha, \Omega_{3}}
$$

is finite.

In this definition $|f|_{k, \alpha, \Omega}$ denotes the usual Hölder norm of the function $f$ over the (bounded) open set $\Omega$.

The one result about these we shall use frequently, and without comment, is that to check if a function $u$ is an element of some $\mathcal{C}_{\nu}^{0, \alpha}$, say, it is sufficient to check that $d(x)^{-\nu}|u(x)| \leq C$ and $d(x)^{-\nu-1}|\nabla u(x)| \leq C$. Here $d(x)$ is a smooth positive function on the complement of $\Sigma$, equaling $\left|x-x_{i}\right|$ on each $B\left(x_{i}, 1\right)$. 


\section{The linearized operator on the unit ball}

In this section we shall study the Dirichlet problem in the unit ball $B(0,1)$ for the linearization about one of the radial Delaunay solutions $u_{\varepsilon}(R, 0, x)$, i.e. when $a=0$. We restrict to the radial Delaunay solutions because they may be studied using separation of variables. Later we shall use a perturbation argument to treat the more general case.

For simplicity, denote the linearization $L_{\varepsilon, R, 0}$ by $L_{\varepsilon, R}$, and when $R=1$, simply by $L_{\varepsilon}$. Our goal here is to study the problem

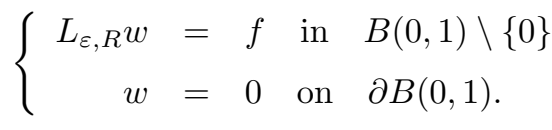

Specifically, we wish to find suitable spaces on which $L_{\varepsilon, R}$ is surjective, and to find optimal estimates for the solution of (19) in these spaces, uniformly as $\varepsilon$ tends to zero. The first part of this was already considered in [7]. There, it was proved that $L_{\varepsilon, R}$ is surjective as a map between certain weighted Sobolev spaces, but the uniformity in $\varepsilon$ was not considered. For various technical reasons, it is more useful here to consider the action of $L_{\varepsilon, R}$ on the family of weighted Hölder spaces defined in the previous section.

One can prove, analogous to the result of [7], that

$$
L_{\varepsilon, R}: \mathcal{C}_{\mu, \mathcal{D}}^{2, \alpha}(B(0,1) \backslash\{0\}) \longrightarrow \mathcal{C}_{\mu-2}^{0, \alpha}(B(0,1) \backslash\{0\})
$$

is Fredholm for all $\mu \in \mathbb{R}, \mu \notin\left\{\mu_{j}^{ \pm}(\varepsilon)\right\}$, where $\left\{\mu_{j}^{ \pm}(\varepsilon)\right\}$ is a discrete set, with each $\mu_{j}^{ \pm}(\varepsilon)$ determined by the ordinary differential equation induced by $L_{\varepsilon, R}$ on the $j$ th eigenspace of the Laplacian on $S^{N-1}$. These weights $\mu_{j}(\varepsilon)$ correspond to the growth rates of solutions of $L_{\varepsilon, R} w=0$ near 0 . Their values depend on $\varepsilon$ in general, although fortunately those with $j=0, \ldots, N$ do not. This is because the solutions of $L_{\varepsilon, R} w=0$ with low cross-sectional eigenvalue are given quite explicitly and geometrically, as we show below, and their growth rates do not depend on $\varepsilon$. These values play the same role as the the indicial roots of a regular singular problem. However, their values for $j>N$ are determined in a far less explicit manner, analytically rather than algebraically, and so are usually impossible to compute. As we have just noted, we know the solutions of this equation, hence the rates of growth of these solutions, explicitly when $j=0, \ldots, N$, so we can determine, as in [7], that $\mu_{0}^{ \pm}=(2-N) / 2$ and $\mu_{j}^{+}=(4-N) / 2, \mu_{j}^{-}=-N / 2, j=1, \ldots, N$. From this, one sees that

$$
\Psi_{\varepsilon, R, a}^{0,+} \in \mathcal{C}_{\mu}^{2, \alpha} \quad \text { for all } \mu \leq \frac{2-N}{2}, \quad \Psi_{\varepsilon, R, a}^{0,-} \in \mathcal{C}_{\mu}^{2, \alpha} \quad \text { for all } \mu<\frac{2-N}{2},
$$

and for $j=1, \ldots, N$,

$$
\Psi_{\varepsilon, R, a}^{j,+} \in \mathcal{C}_{\mu}^{2, \alpha} \quad \text { for all } \mu \leq \frac{4-N}{2}, \quad \Psi_{\varepsilon, R, a}^{j,-} \in \mathcal{C}_{\mu}^{2, \alpha} \quad \text { for all } \mu \leq \frac{-N}{2} .
$$

As we pointed out above, the values $\mu_{j}^{ \pm}$, for $j=0, \ldots, N$, do not depend on $\varepsilon$.

It turns out that (20) is surjective when $\mu<(2-N) / 2, \mu \neq \mu_{j}^{-}$, and injective when $\mu>$ $(2-N) / 2$. The basic conflict is that, although this indicates that we should be working on spaces with weight less than $(2-N) / 2$, we are using $L_{\varepsilon, R}$ to correct the approximate solution, which only grows like $r^{(2-N) / 2}$, and we obviously do not want the correction term to blow up faster than this. As proved in [7], this may be remedied by working in a finite dimensional extension of $\mathcal{C}_{\mu}^{2, \alpha}$, where $(2-N) / 2<\mu<(4-N) / 2$ and the extension is the span of the Jacobi fields $\Psi_{\varepsilon, R}^{0, \pm}$. The point is that although in general (19) has no solution $w \in \mathcal{C}_{\mu}^{2, \alpha}$ when $f \in \mathcal{C}_{\mu-2}^{0, \alpha}$ and $\mu>(2-N) / 2$, there is a solution with the correct decay but with eigencomponent $w_{0}$ having the wrong Dirichlet data. To correct this, one may add on some multiple of one or the other of the 
Jacobi fields $\Psi_{\varepsilon, R}^{0, \pm}$. We shall prove something similar, but with two additional considerations. The first is that unlike the analogous situation in [6], any choice of right inverse has norm blowing up as $\varepsilon$ tends to zero. However, this blow-up happens only on the subspace of functions spanned by the eigenfunctions $\phi_{j}(\theta), j=0, \ldots, N$. In order to obtain reasonable estimates, we need to work in the space with weight $\mu$ with $1<\mu<2$. For this reason we shall also need to append to this 'deficiency space' the Jacobi fields with index $j=1, \ldots, N$. The other consideration is that we wish to prove surjectivity using only the Jacobi fields $\Psi_{\varepsilon, R}^{j,+}$, because the others are considerably more difficult to use in the nonlinear analysis. In fact, using the Jacobi fields $\Psi_{\varepsilon, R}^{j,-}$ would force us to reposition the singularities or change the Delaunay parameters, which for technical reasons it is far better to avoid. On the level of linear analysis this is a good choice as well, for with this restricted domain the linearization is an isomorphism rather than just surjective.

We come to the main result of this section.

Proposition 5 Fix $R \in \mathbb{R}^{+}, R \neq 1$, and $\mu \in((4-N) / 2,2)$ and define the deficiency space

$$
W \equiv \operatorname{span}\left\{\Psi_{\varepsilon, R}^{j,+}: j=0, \ldots, N\right\} .
$$

Then there exists an $\varepsilon_{0}>0$ such that for all $\varepsilon \in\left(0, \varepsilon_{0}\right]$, the operator

$$
L_{\varepsilon, R}:\left[\mathcal{C}_{\mu}^{2, \alpha}(B(0,1) \backslash\{0\})\right]_{\mathcal{D}} \oplus W \longrightarrow \mathcal{C}_{\mu-2}^{0, \alpha}(B(0,1) \backslash\{0\})
$$

is an isomorphism (the subscript $\mathcal{D}$ referes to the fact that we only consider the subspace of those functions which vanish at the boundary $\partial B(0,1))$. The inverse map will be denoted $L_{\varepsilon, R}^{-1}$. In particular, for $f \in \mathcal{C}_{\mu-2}^{0, \alpha}(B(0,1) \backslash\{0\})$ there exists a unique solution $w$ of (19) which has a decomposition

$$
w(x)=G_{\varepsilon, R}(f)(x)+\sum_{j=0}^{N} K_{\varepsilon, R}^{j}(f) \frac{1}{\varepsilon} \Psi_{\varepsilon}^{j,+}(x / R) .
$$

The operator

$$
G_{\varepsilon, R}: \mathcal{C}_{\mu-2}^{0, \alpha}(B(0,1) \backslash\{0\}) \rightarrow \mathcal{C}_{\mu}^{2, \alpha}(B(0,1) \backslash\{0\})
$$

and functionals

$$
K_{\varepsilon, R}^{j}: \mathcal{C}_{\mu-2}^{0, \alpha}(B(0,1) \backslash\{0\}) \rightarrow \mathbb{R}
$$

satisfy the following properties:

- $G_{\varepsilon, R}$ is defined for $\mu \in((4-N) / 2,2)$ and is bounded independently of $\varepsilon$ when $1<\mu<2$.

- The restriction of $G_{\varepsilon, R}$ to the space of functions $h(r, \theta)$ with eigencomponents $h_{j}(r)$ vanishing for $j=0, \ldots, N$, is defined and bounded independently of $\varepsilon$ when $-N<\mu<2$.

- The functional $K_{\varepsilon, R}^{0}$ is defined for all $\mu>(2-N) / 2$ and is bounded independently of $\varepsilon$ when $0<\mu$.

- The functionals $K_{\varepsilon, R}^{j}, j=1, \ldots, N$, are defined for all $\mu>(4-N) / 2$ and are bounded independently of $\varepsilon$ when $1<\mu$.

The proof of Proposition 5 will be broken into several steps. The solution is constructed by separation of variables, restricting the problem to each eigenspace of the Laplacian on the sphere. First, we note that although the parameter $R$ is important later, at this stage it is irrelevant and may be scaled away by setting $y=x / R$. In fact, replacing $f(x)$ by $R^{-2} f(x / R)$ and $w(x)$ by $w(x / R)$, we see that (19) is equivalent to

$$
\left\{\begin{aligned}
L_{\varepsilon} w= & \text { in } \quad B(0,1 / R) \backslash\{0\} \\
w=0 & \text { on } \quad \partial B(0,1 / R) .
\end{aligned}\right.
$$


When we change to the variable $t=-\log |x|$, this rescaling has the effect of replacing $v_{\varepsilon, R}(t)=$ $v_{\varepsilon}(t+\log R)$ by $v_{\varepsilon}(t)$. The $K_{\varepsilon, R}^{j}$ will be denoted simply $K_{\varepsilon}^{j}$, and so on. In the $(t, \theta)$ variables, the Hölder spaces are converted to the ordinary (translation-invariant) spaces, with the weight function $e^{-\mu t}$.

Write the eigenfunction decompositions of $w$ and $f$ as

$$
w(x)=|x|^{-\frac{N-2}{2}} \sum_{j=0}^{\infty} w_{j}(-\log |x|) \phi_{j}(\theta), \quad \text { and } \quad f(x)=|x|^{-\frac{N+2}{2}} \sum_{j=0}^{\infty} f_{j}(-\log |x|) \phi_{j}(\theta) .
$$

If $w$ solves $(25)$ then $w_{j}(t)$ solves

$$
\left\{\begin{aligned}
\mathbb{L}_{\epsilon, j} w_{j}(t) & =\frac{d^{2} w_{j}}{d t^{2}}-\frac{(N-2)^{2}}{4} w_{j}-\lambda_{j} w_{j}+\frac{N(N+2)}{4} v_{\varepsilon}^{\frac{4}{N-2}} w_{j}=f_{j} \\
w_{j}(\log R) & =0 .
\end{aligned}\right.
$$

The $\mathbb{L}_{\epsilon, j}$ are the restrictions to the eigenspaces of $\Delta_{S^{N-1}}$ of the operator

$$
\mathbb{L}_{\epsilon} w(t, \theta)=\frac{d^{2} w}{d t^{2}}-\frac{(N-2)^{2}}{4} w+\Delta_{S^{N-1}} w+\frac{N(N+2)}{4} v_{\varepsilon}^{\frac{4}{N-2}} w,
$$

which is simply problem (5) transformed from $\mathbb{R}^{N} \backslash\{0\}$ to the cylinder $\mathbb{R} \times S^{N-1}$.

We may assume, after multiplying by a suitable factor, that

$$
\|f\|_{0, \alpha, \mu-2}=1 .
$$

We first study the operators $\mathbb{L}_{\epsilon, j}$ for $j>N$, then for $j=0$ and finally for $j=1, \ldots, N$.

Step 1. Suppose that $-N<\mu<2$. Let $\bar{f}$ be the projection of $f$ onto the sum of the eigenspaces of $\Delta_{S^{N-1}}$ with $j>N$,

$$
\bar{f}(t, \theta)=\sum_{j \geq N+1} f_{j}(t) \phi_{j}(\theta)
$$

We must solve

$$
\left\{\begin{aligned}
\mathbb{L}_{\epsilon} \bar{w}(t, \theta) & =\bar{f}(t, \theta) \quad \text { in } \quad(\log R,+\infty) \times S^{N-1} \\
\bar{w}(\log R, \theta) & =0 .
\end{aligned}\right.
$$

To do this we first show that for each $T>\log R$ there exists a unique solution of

$$
\left\{\begin{aligned}
\mathbb{L}_{\epsilon} \bar{w}_{T}(t, \theta) & =\bar{f} \text { in }(\log R, T) \times S^{N-1} \\
\bar{w}_{T}(\log R, \theta) & =0 \\
\bar{w}_{T}(T, \theta) & =0
\end{aligned}\right.
$$

This solution can be obtained variationally. Consider the energy function $\mathcal{E}_{T}$

$$
\mathcal{E}_{T}(w)=\int_{\log R}^{T} \int_{S^{N-1}}\left(\dot{w}^{2}+\frac{(N-2)^{2}}{4} w^{2}+\left|\nabla_{S^{N-1}} w\right|^{2}-\frac{N(N+2)}{4} v_{\varepsilon}^{\frac{4}{N-2}} w^{2}+\bar{f} w\right) d t d \theta .
$$

Since $\lambda_{j} \geq \lambda_{N+1}=2 N$, we estimate

$$
\mathcal{E}_{T}(w) \geq \int_{\log R}^{T} \int_{S^{N-1}}\left(\dot{w}^{2}+\left(\frac{(N+2)^{2}}{4}-\frac{N(N+2)}{4} v_{\varepsilon}^{\frac{4}{N-2}}\right) w^{2}+\bar{f} w\right) d t d \theta .
$$

Since $0<v_{\varepsilon}<1$, this energy is convex and proper, and the existence of a unique minimizer for $\mathcal{E}_{T}$ is immediate. 
Lemma 1 For $-N<\mu<2$, let $\delta=\frac{N-2}{2}+\mu$. Then, for all $R>0$, there exist constants $\varepsilon_{0}>0$ and $C>0$ independent of $T$, such that for all $\varepsilon \in\left(0, \varepsilon_{0}\right]$, we have

$$
\sup _{\theta \in S^{N-1}} \sup _{t \in[\log R, T]} e^{\delta t}\left|\bar{w}_{T}(t, \theta)\right| \leq C .
$$

Proof : We prove this by contradiction. Recall that, by assumption,

$$
\sup _{\theta \in S^{N-1}} \sup _{t \in[\log R, T]} e^{\delta t}|\bar{f}(t, \theta)| \leq 1 .
$$

If the assertion were not true, then there would exist sequences of functions $\bar{f}_{i}$, numbers $T_{i}$ and Delaunay parameters $\varepsilon_{i}$ and a sequence of solutions $\bar{w}_{T_{i}}$ such that

$$
\lim _{i \rightarrow \infty}\left(\sup _{\theta \in S^{N-1}} \sup _{R \leq t \leq T_{i}} e^{\delta t}\left|\bar{w}_{T_{i}}(t, \theta)\right|\right)=\infty .
$$

We let $v_{i}=v_{\varepsilon_{i}}$. Now choose $t_{i} \in\left(\log R, T_{i}\right)$ such that

$$
\sup _{\theta \in S^{N-1}} e^{\delta t_{i}}\left|\bar{w}_{T_{i}}\left(t_{i}, \theta\right)\right|=\sup _{\theta \in S^{N-1}} \sup _{\log } e_{\leq t \leq T_{i}} e^{\delta t}\left|\bar{w}_{T_{i}}(t, \theta)\right| \equiv A_{i},
$$

and define

$$
\begin{gathered}
\tilde{w}_{i}(t, \theta)=A_{i}^{-1} e^{\delta t_{i}} \bar{w}_{T_{i}}\left(t+t_{i}, \theta\right), \\
\tilde{f}_{i}(t, \theta)=A_{i}^{-1} e^{\delta t_{i}} \bar{f}\left(t+t_{i}, \theta\right) .
\end{gathered}
$$

Then, by definition,

$$
\sup _{\theta \in S^{N-1}} \sup _{\log R-t_{i} \leq t \leq T_{i}-t_{i}} e^{\delta t}\left|\tilde{w}_{i}(t, \theta)\right|=1,
$$

and $\tilde{f}_{i}$ tends to 0 in norm. In addition,

$$
\frac{d^{2} \tilde{w}_{i}}{d t^{2}}-\frac{(N-2)^{2}}{4} \tilde{w}_{i}+\Delta_{S^{N-1}} \tilde{w}_{i}+\frac{N(N+2)}{4} v_{i}^{\frac{4}{N-2}}\left(t+t_{i}\right) \tilde{w}_{i}=\tilde{f}_{i},
$$

on $\left[\log R-t_{i}, T_{i}-t_{i}\right] \times S^{N-1}$. Passing to a subsequence, we may assume that $\log R-t_{i}$ converges to some number $\tau_{1} \in \mathbb{R}^{-} \cup\{-\infty\}$ and also that $T_{i}-t_{i}$ converges to $\tau_{2} \in \mathbb{R}^{+} \cup\{+\infty\}$. Notice that the sequences $t_{i}-\log R$ and ${ }_{i}^{T}-t_{i}$ remain bounded away from 0 . For example, i follows from the hypothesis that $\frac{d^{2} \tilde{w}_{i}}{d t^{2}}+\Delta_{S^{N-1}} \tilde{w}_{i}$ is uniformly bounded by a constant times $e^{\delta t_{i}}$ in $(\log R-$ $\left.t_{i}, \log R-t_{i}+2\right) \times S^{N-1}$ and so does $\tilde{w}_{i}$. In addition $\tilde{w}_{i}$ is equal to 0 on $\left\{\log R-t_{i}\right\} \times S^{N-1}$. Thus we also have $\nabla \tilde{w}_{i}$ which is bounded by a constant times $e^{\delta t_{i}}$ in $\left(\log R-t_{i}, \log R-t_{i}+1\right) \times S^{N-1}$. And this proves that $t_{i}-\log R$ remains bounded away from 0 . Furthermore, we may also assume that over every compact set of $\left(\tau_{1}, \tau_{2}\right)$, the sequence $v_{i}\left(t+t_{i}\right)$ converges to $v_{\infty}(t)$ and $\tilde{w}_{i}$ converges to $\tilde{w}$. By construction, $\tilde{w} \neq 0$. Then $v_{\infty}$ is a solution of $(6)$ and $\tilde{w}$ satisfies the equation

$$
\frac{d^{2} \tilde{w}}{d t^{2}}-\frac{(N-2)^{2}}{4} \tilde{w}+\Delta_{S^{N-1}} \tilde{w}+\frac{N(N+2)}{4} v_{\infty}^{\frac{4}{N-2}} \tilde{w}=0
$$

on $\left[\tau_{1}, \tau_{2}\right] \times S^{N-1}$. Let $H_{\infty}=H\left(v_{\infty}, \dot{v}_{\infty}\right)$. There are a few cases to consider, depending on the values of $\tau_{1}, \tau_{2}$ and $H_{\infty}$. To analyze these, we require some growth estimates.

Lemma 2 For every $\eta>0$, there exists $\varepsilon_{0}>0$ such that, for all $j \geq N+1$ and for all $\varepsilon \in\left(0, \varepsilon_{0}\right]$, every solution of

$$
\frac{d^{2} w}{d t^{2}}-\left(\frac{(N-2)^{2}}{4}+\lambda_{j}-\frac{N(N+2)}{4} v_{\varepsilon}^{\frac{4}{N-2}}\right) w=0
$$

either decays to 0 faster than $e^{-\left(\frac{(N+2)^{2}}{4}-4 \eta\right)^{1 / 2} t}$ at $\infty$ (respectively, $e^{\left(\frac{(N+2)^{2}}{4}-4 \eta\right)^{1 / 2} t}$ at $-\infty$ ) or blows up faster than $e^{\left(\frac{(N+2)^{2}}{4}-4 \eta\right)^{1 / 2} t}$ at $\infty$ (respectively, $e^{-\left(\frac{(N+2)^{2}}{4}-4 \eta\right)^{1 / 2} t}$ at $\left.-\infty\right)$. 
Proof : Because $j \geq N+1$, the term of order zero, $\frac{(N-2)^{2}}{4}+\lambda_{j}-\frac{N(N+2)}{4} v_{\varepsilon}^{\frac{4}{N-2}}$ is positive, hence $\mathbb{L}_{\epsilon, j}$ satisfies the maximum principle. Let

$$
\delta_{j}=\left(\frac{(N-2)^{2}}{4}+\lambda_{j}-\frac{N(N+2)}{4}\right)^{1 / 2} \quad \text { and } \quad \beta(\eta)=\left(\frac{(N+2)^{2}}{4}-\eta\right)^{1 / 2} .
$$

We shall show the existence of a solution bounded above by $e^{-\beta(2 \eta) t}$ as $t$ tends to $\infty$ and bounded below by $e^{-\beta(2 \eta) t}$ as $t$ tends to $-\infty$. Replacing $t$ by $-t$ yields a solution with the appropriate exponential decay at $-\infty$ and blowup at $\infty$. Since these span the space of all solutions, this will prove the lemma.

Since $e^{-\delta_{j} t}$ satisfies $\mathbb{L}_{\epsilon, j} e^{-\delta_{j} t} \leq 0$, it is elementary that there exists a function $y_{j}(t)$ such that $y_{j}(0)=1$ and

$$
y_{j}(t) \geq e^{-\delta_{j} t} \quad \text { for } \quad t \leq 0, \quad 0 \leq y_{j}(t) \leq e^{-\delta_{j} t} \quad \text { for } \quad t \geq 0 .
$$

Next, note that

$$
y_{j}\left(t+T_{\varepsilon}\right)=\frac{y_{j}\left(T_{\varepsilon} / 2\right)}{y_{j}\left(-T_{\varepsilon} / 2\right)} y_{j}(t) \quad \text { for all } t
$$

To see this, simply observe that $w(t) \equiv y_{j}\left(T_{\varepsilon} / 2\right) y_{j}(t)-y_{j}\left(t+T_{\varepsilon}\right) y_{j}\left(-T_{\varepsilon} / 2\right)$ solves $(28)$, decays exponentially at $\infty$ and takes the value 0 at $-T_{\varepsilon} / 2$, hence by the maximum principle must vanish identically. We next claim that

$$
0<y_{j}(t) \leq e^{-\beta(4 \eta) t} \quad \text { for } \quad t \in\left[0, T_{\varepsilon} / 2\right], \quad \text { and } y_{j}(t) \geq e^{-\beta(4 \eta) t} \quad \text { for } \quad t \in\left[-T_{\varepsilon} / 2,0\right] .
$$

Granting this for the moment, then

$$
\frac{y_{j}\left(T_{\varepsilon} / 2\right)}{y_{j}\left(-T_{\varepsilon} / 2\right)} \leq \frac{e^{-\beta(4 \eta) T_{\varepsilon} / 2}}{e^{\beta(4 \eta) T_{\varepsilon} / 2}}=e^{-\beta(4 \eta) T_{\varepsilon}} .
$$

Using this, along with (29) and (30), the desired estimates for $y_{j}(t)$ for all $t$ are immediate. Hence, to prove the lemma, it will suffice to examine the function $y_{j}$ on the interval $\left[-T_{\varepsilon} / 2, T_{\varepsilon} / 2\right]$.

From Proposition 2, there exist $\varepsilon_{0}>0, t_{0}>0$ such that for all $\varepsilon \in\left(0, \varepsilon_{0}\right]$ and $t \in\left[-T_{\varepsilon} / 2+\right.$ $\left.t_{0}, T_{\varepsilon} / 2-t_{0}\right]$

$$
\frac{(N-2)^{2}}{4}+\lambda_{j}-\frac{N(N+2)}{4} v_{\varepsilon}^{\frac{4}{N-2}}(t) \geq \frac{(N+2)^{2}}{4}-\eta=\beta(\eta)^{2} .
$$

For simplicity in the notations, we set $T=T_{\varepsilon} / 2-t_{0}$. We choose $a, b \in \mathbb{R}$ such that $z(t) \equiv$ $a e^{\beta(\eta) t}+b e^{-\beta(\eta) t}$ satisfies $z(0)=y_{j}(0)=1$ and $z(T)=y_{j}(T)$. Explicitely, we have

$$
a=\frac{y_{j}(T)-e^{-\beta(\eta) T}}{e^{\beta(\eta) T}-e^{-\beta(\eta) T}} \quad \text { and } \quad b=\frac{e^{\beta(\eta) T}-y_{j}(T)}{e^{\beta(\eta) T}-e^{-\beta(\eta) T}}
$$

Thanks to (31), we may use the maximum principle to prove that $y_{j} \leq z$ on $[0, T]$. We are going to show that $y_{j}(T) \leq 2 e^{-\beta(\eta) T}$.

The function $y_{j}$ being strictly decreasing, we have $y_{j}(T)<1$ and thus we find that $b>0$. We may as well assume that $y_{j}(T)>e^{-\beta(\eta) T}$, otherwize there is nothing to prove. Under such an assumption $a>0$ and thus $a, b \in(0,1)$.

Still using the fact that $y_{j}$ is strictly decreasing we find that

$$
y_{j}(T) \leq \min _{t \in[0, T]} z(t)
$$

But, the infimum of $z$ over $\mathbb{R}$ is achieved at the point $t_{m} \geq 0$ which satisfies $e^{2 \beta(\eta) t_{m}}=b / a$. First we rule out the case $t_{m}<T$. Indeed, in this case $y_{j}(T)<z\left(t_{m}\right)=2 \sqrt{a b}$. Introducing in this inequality the expressions for both $a$ and $b$, we find that

$$
\cosh ^{2}(\beta(\eta) T) y_{j}^{2}(T)-2 \cosh (\beta(\eta) T) y_{j}(T)+1<0,
$$


which is not possible. Therefore, we always have $t_{m} \geq T$ and this implies that

$$
a=b e^{-2 \beta(\eta) t_{m}} \leq b e^{-2 \beta(\eta) T} .
$$

In particular we obtain $($ since $b<1)$

$$
y_{j}(T) \leq z(T)=a e^{\beta(\eta) T}+b e^{-\beta(\eta) T} \leq 2 b e^{-2 \beta(\eta) T} \leq 2 e^{-2 \beta(\eta) T} .
$$

In every case, we conclude that $y_{j}(T) \leq 2 e^{-\beta(\eta) T}$, where we recall that $T=\frac{T_{\varepsilon}}{2}-t_{0}$.

Now, for $\varepsilon$ small enough, we have

$$
2 e^{-\beta(\eta)\left(\frac{T_{\varepsilon}}{2}-t_{0}\right)} \leq e^{-\beta(2 \eta)\left(\frac{T_{\varepsilon}}{2}-t_{0}\right)},
$$

we may then use once more the maximum principle on the interval $\left[0, T_{\varepsilon} / 2-t_{0}\right]$ to conclude that

$$
0 \leq y_{j}(t) \leq e^{-\beta(2 \eta) t}, \quad 0 \leq t \leq T_{\varepsilon} / 2-t_{0}, \quad \text { and } \quad y_{j}(t) \geq e^{-\beta(2 \eta) t}, \quad-T_{\varepsilon} / 2+t_{0} \leq t \leq 0 .
$$

Furthermore, $y_{j}$ is monotone decreasing, so its maximum on any interval is attained at the left endpoint and its minimum at the right endpoint. In addition, for $\varepsilon$ sufficiently small,

$$
e^{-\beta(2 \eta)\left(\frac{T_{\varepsilon}}{2}-t_{0}\right)} \leq e^{-\beta(4 \eta) \frac{T_{\varepsilon}}{2}}
$$

thus $y_{j}(t) \leq e^{-\beta(4 \eta) t}$ for all $t \in\left[0, T_{\varepsilon} / 2\right]$. This completes the proof of $(30)$ and hence the lemma.

We now return to our proof of Lemma 1.

- First consider the case where $H_{\infty} \neq 0$. Decompose $\tilde{w}$ as

$$
\tilde{w}(t, \theta)=\sum_{j \geq N+1} \tilde{w}_{j}(t) \phi_{j}(\theta)
$$

If $\tau_{1}=-\infty$ then, taking $4 \eta \leq \frac{(N+2)^{2}}{4}-\delta^{2}$ in Lemma 2 and using the fact that $\tilde{w}$ is bounded as $t \rightarrow-\infty$ by $e^{-\delta t}$ with $\delta<\frac{N+2}{2}$, we see that for $\varepsilon$ sufficiently small, $\tilde{w}_{j}$ decays exponentially as $t$ goes to $-\infty$. Similarly, if $\tau_{2}=+\infty$, then by the same argument $\tilde{w}_{j}$ decays exponentially as $t \rightarrow \infty$. If $\tau_{1}>-\infty$, then $\tilde{w}_{j}\left(\tau_{1}\right)=0$ and if $\tau_{2}<\infty$, then $\tilde{w}_{j}\left(\tau_{2}\right)=0$.

Therefore, we can multiply the equation satisfied by $\tilde{w}_{j}$ by $\tilde{w}_{j}$ itself and integrate by parts. There are no boundary terms because of the exponential decay and vanishing boundary values, so we obtain

$$
0=\int_{\tau_{1}}^{\tau_{2}}\left(\dot{\tilde{w}}_{j}^{2}+\frac{(N-2)^{2}}{4} \tilde{w}_{j}^{2}+\lambda_{j} \tilde{w}_{j}^{2}-\frac{N(N+2)}{4} v_{\infty}^{\frac{4}{N-2}} \tilde{w}_{j}^{2}\right) d t
$$

But since $\lambda_{j} \geq 2 N$ and $0<v_{\infty}<1$, we conclude that $\tilde{w} \equiv 0$.

- Next consider the case where $H_{\infty}=0$. This case is almost identical to the last one, except that the exponential decay is simpler to obtain. Decompose $\tilde{w}$ as before. If $\tau_{1}=-\infty$ then by Proposition 2 and (27) $\tilde{w}_{j}$ grows asymptotically like $e^{ \pm \gamma_{j} t}$, where $\gamma_{j}^{2}=\frac{(N-2)^{2}}{4}+\lambda_{j}$, near $-\infty$. But $\tilde{w}$ is bounded by $e^{-\delta t}$ near $-\infty$, where $\delta<\frac{N+2}{2} \leq \gamma_{j}$, and so $\tilde{w}_{j}$ decays like $e^{\gamma_{j} t}$ at $-\infty$. Similarly, if $\tau_{2}=+\infty, \tilde{w}_{j}$ decays exponentially as $t$ goes to $+\infty$, and as before, $\tilde{w}_{j}\left(\tau_{1}\right)=0$ and $\tilde{w}_{j}\left(\tau_{2}\right)=0$ if either $\tau_{1}$ or $\tau_{2}$ are finite.

The same integration by parts as before implies that $\tilde{w} \equiv 0$. 
In either case, we have produced a contradiction, since by assumption $\tilde{w}$ is non trivial. The proof of Lemma 1 is complete.

Finally, we let $T \rightarrow+\infty$ to get the existence of a unique solution $\bar{w}$ to (26) which is uniformly bounded by $C e^{-\delta t}$ for $t \geq 0$.

The second and third steps of the proof of Proposition 5 are rather similar to Step 1 above. The main difference is that an extra step is needed to ensure that $w$ has vanishing boundary value at $t=\log R$. This is where the nonuniformity of $L_{\varepsilon, R}^{-1}$ as $\varepsilon \rightarrow 0$ arises.

Step 2. Let $j=0$ and assume that $\mu>0$. We shall now solve the equation

$$
\left\{\begin{array}{l}
\mathbb{L}_{\varepsilon, 0} w_{0}(t)=f_{0} \quad \text { in } \quad(\log R, \infty) \\
w_{0}(\log R)=0
\end{array}\right.
$$

Normalize $f_{0}$ so that $\left\|f_{0}\right\|_{0, \alpha, \mu-2}=1$, and choose an extension $\tilde{f}_{0}$ of $f_{0}$ to $\mathbb{R}$ with the property that $\left\|\tilde{f}_{0}\right\|_{0, \alpha, \mu-2} \leq 2$. For each $T>\log R$, let $w_{T}$ be the unique solution of

$$
\mathbb{L}_{\varepsilon, 0} w_{T}=\tilde{f}_{0}, \quad w_{T}(T)=0, \quad \dot{w}_{T}(T)=0 .
$$

Lemma 3 For $\mu>0$, let $\delta=\frac{N-2}{2}+\mu$. Then for all $R>0$, there are constants $\varepsilon_{0}>0$ and $C>0$ independent of $T$, such that for all $\varepsilon \in\left(0, \varepsilon_{0}\right]$ we have

$$
\sup _{t \in(-\infty, T]} e^{\delta t}\left|\bar{w}_{T}(t)\right| \leq C
$$

Proof : First, the two independent solutions of $\mathbb{L}_{\varepsilon, 0} w=0$ are the functions $\Phi_{\varepsilon}^{0, \pm}=r^{\frac{N-2}{2}} \Psi_{\varepsilon}^{0, \pm}$, and neither these, nor any linear combinations of them decay or grow exponentially. In fact, $\Phi_{\varepsilon}^{0,+}$ is periodic (of period $T_{\varepsilon}$ ), while $\Phi_{\varepsilon}^{0,-}$ grows linearly. This last fact may be seen by differentiating the equality $v_{\varepsilon}\left(t+T_{\varepsilon}\right)=v_{\varepsilon}(t)$ with respect to $\varepsilon$ to get

$$
\Phi_{\varepsilon}^{0,-}\left(t+T_{\varepsilon}\right)+\frac{d T_{\varepsilon}}{d \varepsilon} \Phi_{\varepsilon}^{0,+}\left(t+T_{\varepsilon}\right)=\Phi_{\varepsilon}^{0,-}(t) ;
$$

this gives the linear growth rate since the derivative of $T_{\varepsilon}$ never vanishes. (Even without knowing that $T_{\varepsilon}$ is never stationary, it is elementary that no solutions of $\mathbb{L}_{\varepsilon, 0}$ can decay at infinity.)

The remainder of the proof is nearly identical to that of Lemma 1. If the claim were not true, then there would exist sequences $f_{0, i}, T_{i}, \varepsilon_{i}$ and $\bar{w}_{T_{i}}$ such that $A_{i} \equiv \sup _{t \in\left(-\infty, T_{i}\right]} e^{\delta t}\left|\bar{w}_{T_{i}}\right|$ tends to infinity. Notice that $\bar{w}_{T_{i}}$ grows at most linearly as $t$ tends to $-\infty$, hence the supremum in the last fomula is always achieved. Choosing $t_{i} \in\left(-\infty, T_{i}\right)$ to maximize $e^{\delta t}\left|\bar{w}_{T_{i}}(t)\right|$ (so that $\left.e^{\delta t_{i}}\left|\bar{w}_{T_{i}}\left(t_{i}\right)\right|=A_{i}\right)$, we rescale the functions and translate the independent variable by $t_{i}$ to obtain a solution of

$$
\frac{d^{2} \tilde{w}_{i}}{d t^{2}}-\frac{(N-2)^{2}}{4} \tilde{w}_{i}+\frac{N(N+2)}{4} v_{i}^{\frac{4}{N-2}} \tilde{w}_{i}=\tilde{f}_{0, i}
$$

in $\left(-\infty, T_{i}-t_{i}\right]$. This solution satisfies

$$
\sup _{t \in\left(-\infty, T_{i}-t_{i}\right]} e^{\delta t}\left|\tilde{w}_{i}(t)\right|=1
$$

while $\tilde{f}_{0, i}$ tends to zero in norm. Again, the sequence $T_{i}-t_{i}$ is bounded away from 0 .

Passing to a subsequence, we obtain a nontrivial solution $\tilde{w}$ of the equation

$$
\frac{d^{2} \tilde{w}}{d t^{2}}-\frac{(N-2)^{2}}{4} \tilde{w}+\frac{N(N+2)}{4} v_{\infty}^{\frac{4}{N-2}} \tilde{w}=0
$$

over $\left(-\infty, \tau_{2}\right]$ 
- We can immediately see that $\tau_{2}=\infty$, for if not then $\tilde{w}$ would be a non trivial solution of (33) such that $\tilde{w}\left(\tau_{2}\right)=\dot{\tilde{w}}\left(\tau_{2}\right)=0$, which would imply that $\tilde{w} \equiv 0$.

- We can also easily rule out the case where $H\left(v_{\infty}, \dot{v}_{\infty}\right) \equiv H_{\infty} \neq 0$. For if $\tilde{w}$ were a nontrivial solution of (33) then it would decay exponentially at $\infty$. But we have already seen that no solutions of $\mathbb{L}_{\varepsilon, 0} w=0$ decays exponentially.

- The final case, where $H_{\infty}=0$, is also easy to rule out. By Proposition 2 and equation (33), $\tilde{w}$ grows asymptotically near $\infty$ like $e^{ \pm \frac{N-2}{2} t}$. Since $\tilde{w}$ is bounded by $e^{-\delta t}$ and because $\delta>\frac{N-2}{2}$, we may conclude that $\tilde{w} \equiv 0$.

Remark 4 If, in the statement of Lemma 3, we consider $\varepsilon$ to be fixed, then, in the proof of this result we only have to rule out the first two points. In which case, it is enough to assume that $\delta>0$ (or equivalently $\mu>\frac{2-N}{2}$ ).

With the proof of Lemma 3 complete, we let $T \rightarrow \infty$ and obtain a unique solution $\bar{w}$ to (32) which satisfies

$$
\sup _{t \in[\log R,+\infty)} e^{\delta t}|\bar{w}(t)| \leq C .
$$

There is no reason why this solution should satisfy the boundary condition at $t=\log R$, and so we must add an additional term to correct the boundary data. We define the solution $w_{0}$ by

$$
w_{0}(t)=\bar{w}(t)-\bar{w}(\log R)\left(\Phi_{\varepsilon}^{0,+}(\log R)\right)^{-1} \Phi_{\varepsilon}^{0,+}(t) .
$$

This obviously satisfies the equation and the correct boundary conditions, so the proof will be complete if we show that $\frac{1}{\varepsilon} \Phi_{\varepsilon}^{0,+}(\log R)$ is bounded from below by some constant independent of $\varepsilon$. The following result is deduced easily from Proposition 3 .

Lemma 4 Given $R>0, R \neq 1$, there exists a constant $m_{0}>0$ such that for all $\varepsilon \in\left(0, \varepsilon_{0}\right]$, we have

$$
\Phi_{\varepsilon}^{0,+}(\log R) \geq \varepsilon m_{0} .
$$

Remark 5 The definition of $w_{0}$ is certainly not unique, for we could have added any appropriate combination of $\Phi_{\varepsilon}^{0,-}$ and $\Phi_{\varepsilon}^{0,+}$ to correct the boundary data. However, $\Phi_{\varepsilon}^{0,-}$ is more difficult to use in the nonlinear analysis, so it is important that we only use $\Phi_{\varepsilon}^{0,+}$ here. Moreover, the use of $\Phi_{\varepsilon}^{0,-}$ would oblige us to change the Delaunay parameters of our approximate solution in order to get a solution of the nonlinear problem.

Step 3. Finally, suppose that $1<\mu$. We wish to solve, for $j=1, \ldots, N$, the problem

$$
\left\{\begin{array}{l}
\mathbb{L}_{\epsilon, j} w_{j}(t)=f_{j} \quad \text { in } \quad(\log R, \infty) \\
w_{j}(\log R)=0 .
\end{array}\right.
$$

This is done, mutatis mutandis, following the proofs in Steps 1 and 2. We describe the minor changes that need to be made. First, define an extension $\tilde{f}_{j}$ of $f_{j}$ to all of $\mathbb{R}$, and find the unique solution $\bar{w}_{T}$ of

$$
\mathbb{L}_{\epsilon, j} \bar{w}_{T}=\tilde{f}_{j}, \quad \bar{w}_{T}(T)=\dot{\bar{w}}_{T}(T)=0 .
$$

The uniform bound of $e^{\delta t}\left|\bar{w}_{T}\right|$ on $(-\infty, T]$ is proved as before by contradiction. The fact that that the supremum is achieved follows as before from the fact that, for $t \leq \log R, \bar{w}_{T}$ is a linear combination of $\Phi_{\varepsilon}^{1, \pm}$ and thus blows up as $t$ tends to $-\infty$ at most like a constant times $e^{-t}$. In this step we obtain, by rescaling and translation, a nontrivial solution $\tilde{w}$ of

$$
\frac{d^{2} \tilde{w}}{d t^{2}}-\frac{(N-2)^{2}}{4} \tilde{w}-(N-1) \tilde{w}+\frac{N(N+2)}{4} v_{\infty}^{\frac{4}{N-2}} \tilde{w}=0 .
$$


To rule out the existence of a nontrivial solution to this equation, we reason as before. In the second case, where $H_{\infty} \neq 0$, we use the two explicit solutions of $\mathbb{L}_{\varepsilon, j} w=0$ given by $\Phi_{\varepsilon}^{1, \pm}(t)$. These solutions do grow at an exponential rate, but by hypothesis, both either blow up or do not decay quickly enough, and we conclude as above that this case never occurs. When $H_{\infty}=0$ we note that the asymptotic behaviour of solutions near $\pm \infty$ is now given by $e^{ \pm \frac{N}{2}} t$.

Remark 6 If, in the statement of Lemma 4, we consider $\varepsilon$ to be fixed, in which case, it is enough to assume that $\delta>1$ (or equivalently $\mu>\frac{4-N}{2}$ ) in order to rule out all the different cases.

Now let $T \rightarrow+\infty$ to obtain a suitably bounded solution of (34). Again we must correct the boundary data, so we define, for $j=1, \ldots, N$, the solution $w_{j}$ by

$$
w_{j}(t)=\bar{w}(t)-\bar{w}(\log R)\left(\Phi_{\varepsilon}^{1,+}(\log R)\right)^{-1} \Phi_{\varepsilon}^{1,+}(t) .
$$

Lemma 5 Given $R>0$, there exists a constant $m_{1}>0$ such that for $\varepsilon \in\left(0, \varepsilon_{0}\right]$ and for any $j=1, \ldots, N$, we have $\Phi_{\varepsilon}^{1, \pm}(\log R) \geq m_{1} \varepsilon$.

Proof : Recall that

$$
\Phi_{\varepsilon}^{1,+}(t)=e^{-t}\left(\frac{N-2}{2} v_{\varepsilon}(t)-\frac{\partial v_{\varepsilon}}{\partial t}(t)\right)
$$

By Proposition 3, we get

$$
\Phi_{\varepsilon}^{1,+}(t)=\varepsilon \frac{N-2}{2} e^{-\frac{N}{2} t}+O\left(\varepsilon^{\frac{N+2}{N-2}} e^{\frac{N}{2} t}\right) .
$$

and the result follows.

Again we remark that it will be important in the nonlinear analysis that we have obtained a solution with a $\Phi_{\varepsilon}^{1,+}$, but no $\Phi_{\varepsilon}^{1,-}$, component, for the latter would force the location of the singularities of the exact solution to be different from those of the approximate solution.

Now that we have obtained a solution of the original equation in Proposition 5 and a (weighted) $L^{\infty}$ bound for this solution, estimates for the full Hölder norm follow by standard scaling arguments. This ends the proof of the Proposition.

\section{A model for the linearization about $\bar{u}_{\bar{\varepsilon}}(\bar{R}, \bar{a}, x)$}

In the next two sections we finally study the linearization of $\mathcal{N}$ about the approximate solution $\bar{u}_{\bar{\varepsilon}}(\bar{R}, \bar{a}, x)$ associated to some fixed singular set $\Sigma=\left\{x_{1}, \ldots, x_{n}\right\}$, and parameters $\bar{R}=$ $\left(R_{1}, \ldots, R_{n}\right)$ and admissible $\bar{\varepsilon}=\left(\varepsilon_{1}, \ldots, \varepsilon_{n}\right)$, where each $R_{i}>1$. This last condition can always be assumed since we may dilate our problem by some factor to $\kappa>0$, this will change the set $\Sigma$ into $\left\{\kappa x_{1}, \ldots, \kappa x_{n}\right\}$ and will change the parameters $R_{i}$ into $\kappa R_{i}$ and $a_{i}$ into $a_{i} / \kappa$. As usual, the main point is to analyze this operator uniformly as $\varepsilon$ tends to zero. We shall do this in two steps, first studying the somewhat simpler operator

$$
\mathcal{L}_{\bar{\varepsilon}, \bar{R}} w=\Delta w+\frac{N(N+2)}{4} \sum_{i=1}^{n}\left(\tilde{\chi}\left(x-x_{i}\right) R_{i}^{-2} u_{\varepsilon_{i}}^{\frac{4}{N-2}}\left(\left(x-x_{i}\right) / R_{i}\right)\right) w
$$

where the displacements $a_{i}$ have all been set to zero, and then, in the next section, treating the true linearization as a perturbation of $\mathcal{L}_{\bar{\varepsilon}, \bar{R}}$. Here $\tilde{\chi}$ is a smooth cutoff function equalling $1 \mathrm{in}$ $B(0,1)$, vanishing outside $B(0,2)$, and taking values in $[0,1]$ in the annulus $B(0,2) \backslash B(0,1)$. The point of doing this is that $\mathcal{L}_{\bar{\varepsilon}, \bar{R}}$ is much simpler to study because the term of order zero is radial in each $B\left(x_{i}, 1\right)$. 
The main result is that on suitably weighted function spaces, $\mathcal{L}_{\bar{\varepsilon}, \bar{R}}$ is invertible, with inverse blowing up as $\varepsilon$ tends to zero, but in a manner which we can control precisely. We construct the inverse for $\mathcal{L}_{\bar{\varepsilon}, \bar{R}}$ by solving the equation

$$
\mathcal{L}_{\bar{\varepsilon}, \bar{R}} w=f \quad \text { in } \quad \mathbb{R}^{N} \backslash \Sigma
$$

in three steps: first solve the homogeneous Dirichlet problem for this equation outside the union of the balls $B\left(x_{i}, 1\right)$; next, solve the homogeneous Dirichlet problem for this equation in each of these balls; finally show that the sum of these solutions can be modified to a true solution using the Dirichlet to Neumann maps on the boundaries of these balls.

The main result of this section has a statement parallel to that of Proposition 5:

Proposition 6 Let $\bar{\varepsilon}=\varepsilon \bar{q}$ be an admissible set of Delaunay parameters. Suppose that $\bar{R}=$ $\left(R_{1}, \ldots, R_{n}\right), n \geq 3$, is a collection of numbers, with each $R_{i}>1$, and satisfying (12). Suppose also that

$$
1<\nu<2
$$

Define the deficiency space

$$
\mathcal{W}_{0} \equiv \operatorname{span}\left\{\tilde{\chi}\left(x-x_{i}\right) \Psi_{\varepsilon_{i}}^{j,+}\left(\frac{x-x_{i}}{R_{i}}\right), j=0, \ldots, N\right\}
$$

Then for $\varepsilon$ sufficiently small, the operator

$$
\mathcal{L}_{\bar{\varepsilon}, \bar{R}}: \mathcal{C}_{\nu, 2}^{2, \alpha}\left(\mathbb{R}^{N} \backslash \Sigma\right) \oplus \mathcal{W}_{0} \longrightarrow \mathcal{C}_{\nu-2,-2}^{0, \alpha}\left(\mathbb{R}^{N} \backslash \Sigma\right)
$$

is an isomorphism. In particular, for each $f \in \mathcal{C}_{\nu-2,-2}^{0, \alpha}\left(\mathbb{R}^{N} \backslash \Sigma\right)$ there exists a unique solution of (36) which has a decomposition

$$
w(x)=\mathcal{G}_{\bar{\varepsilon}, \bar{R}}(f)(x)+\sum_{i=1}^{n} \tilde{\chi}\left(x-x_{i}\right) \sum_{j=0}^{N} \mathcal{K}_{\bar{\varepsilon}, \bar{R}, i}^{j}(f) \frac{1}{\varepsilon_{i}} \Psi_{\varepsilon_{i}}^{j,+}\left(\left(x-x_{i}\right) / R_{i}\right),
$$

The operator

$$
\mathcal{G}_{\bar{\varepsilon}, \bar{R}}: \mathcal{C}_{\nu-2,-2}^{0, \alpha}\left(\mathbb{R}^{N} \backslash \Sigma\right) \longrightarrow \mathcal{C}_{\nu, 2}^{2, \alpha}\left(\mathbb{R}^{N} \backslash \Sigma\right)
$$

and functionals

$$
\mathcal{K}_{\bar{\varepsilon}, \bar{R}, i}^{j}: \mathcal{C}_{\nu-2,-2}^{0, \alpha}\left(\mathbb{R}^{N} \backslash \Sigma\right) \longrightarrow \mathbb{R}, \quad j=0, \ldots, N, \quad i=1, \ldots, n
$$

are bounded independently of $\varepsilon$.

We shall denote the right inverse of $\mathcal{L}_{\bar{\varepsilon}, \bar{R}}$ constructed here by $\mathcal{L}_{\bar{\varepsilon}, \bar{R}}^{-1}$. As noted earlier, the proof will be done in several steps.

Step 1 : The exterior problem. Let $\Omega=\mathbb{R}^{N} \backslash \cup_{i=1}^{n} \overline{B\left(x_{i}, 1\right)}$.

Proposition 7 Let $f \in \mathcal{C}_{\nu-2,-2}^{0, \alpha}\left(\mathbb{R}^{N} \backslash \Sigma\right)$. Then for $\varepsilon_{0}>0$ sufficiently small, there exists a unique solution $w \in \mathcal{C}_{\nu, 2}^{2, \alpha}(\Omega)$ to

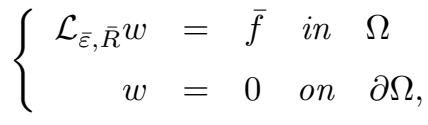

where $\bar{f}$ is the restriction of $f$ to $\Omega$. This solution satisfies the estimate

$$
\|w\|_{2, \alpha, \nu, 2} \leq c\|\bar{f}\|_{0, \alpha, \nu-2,-2},
$$

with some constant $c>0$ independent of $\varepsilon_{i} \in\left(0, \varepsilon_{0}\right]$. The norms here are taken in $\Omega$. 
Proof : The term of order zero in $\mathcal{L}_{\bar{\varepsilon}, \bar{R}}$ has compact support in $\mathbb{R}^{N}$ and, by Proposition 3 , it is bounded by $C \varepsilon$ in $\Omega$. We can transform (39) to a problem of the form $\Delta w^{\prime}+\varepsilon V(x, \bar{\varepsilon}) w^{\prime}=\bar{f}^{\prime}$ in some compact set $\tilde{\Omega}$ with smooth boundary by taking the Kelvin transform at some point outside of $\Omega$. The function $V(x, \bar{\varepsilon})$ is bounded in $\tilde{\Omega}$ independently of $\bar{\varepsilon} . \bar{f} \in \mathcal{C}_{\nu-2,-2}^{0, \alpha}(\Omega)$ implies that $\bar{f}^{\prime} \in \mathcal{C}^{0, \alpha}(\tilde{\Omega})$. The existence and uniform estimate are now standard.

Step 2 : The exterior Dirichlet to Neumann map. We now introduce the exterior Dirichlet to Neumann map. Let $\Psi=\left\{\psi_{1}(\theta), \ldots, \psi_{n}(\theta)\right\} \in \oplus_{i=1}^{n} \mathcal{C}^{2, \alpha}\left(\partial B\left(x_{i}, 1\right)\right)$ be a set of boundary values. It is standard, using Proposition 7 , that there exists a unique solution $\mathcal{C}_{\nu, 2}^{2, \alpha}(\Omega)$ of the homogeneous problem

$$
\left\{\begin{aligned}
\mathcal{L}_{\bar{\varepsilon}, \bar{R}} w=0 & \text { in } \Omega \\
w=\psi_{i} & \text { on } \partial B\left(x_{i}, 1\right), \quad \forall i=1, \ldots, n,
\end{aligned}\right.
$$

which we will denote by $w_{\Psi}$. The correspondence from $\Psi$ to $w_{\Psi}$ is continuous for each $\bar{\varepsilon}$ and $\bar{R}$. Define $\mathcal{S}_{\bar{\varepsilon}, \bar{R}}$ by

$$
\mathcal{S}_{\bar{\varepsilon}, \bar{R}}(\Psi)=\left(\left.\partial_{r_{1}} w_{\Psi}\right|_{r_{1}=1}, \ldots,\left.\partial_{r_{n}} w_{\Psi}\right|_{r_{n}=1}\right) \in \oplus_{i=1}^{n} \mathcal{C}^{1, \alpha}\left(\partial B\left(x_{i}, 1\right)\right),
$$

$r_{i}=\left|x-x_{i}\right|$. This is the Dirichlet to Neumann map for the operator $\mathcal{L}_{\bar{\varepsilon}, \bar{R}}$ on $\Omega$.

Lemma 6 The norms of the mappings

$$
\oplus_{i=1}^{n} \mathcal{C}^{2, \alpha}\left(\partial B\left(x_{i}, 1\right)\right) \ni \Psi \longrightarrow w_{\Psi} \in \mathcal{C}_{\nu, 2}^{2, \alpha}(\Omega)
$$

and

$$
\mathcal{S}_{\bar{\varepsilon}, \bar{R}}: \oplus_{i=1}^{n} \mathcal{C}^{2, \alpha}\left(\partial B\left(x_{i}, 1\right)\right) \longrightarrow \oplus_{i=1}^{n} \mathcal{C}^{1, \alpha}\left(\partial B\left(x_{i}, 1\right)\right),
$$

are bounded independently of $\bar{\varepsilon}$ provided each $\varepsilon_{i}<\varepsilon_{0}$ and $\varepsilon_{0}$ is sufficiently small. Furthermore, if all $\varepsilon_{i}$ tend to $0, \mathcal{S}_{\bar{\varepsilon}, \bar{R}}$ converges to a limiting operator $\mathcal{S}_{0}$ which is the Dirichlet to Neumann map for the Laplacian, $\Delta$, on $\Omega$.

Proof : The boundedness follows from Proposition 7. Convergence of the $\mathcal{S}_{\bar{\varepsilon}, \bar{R}}$ is a consequence of the fact that $\mathcal{L}_{\bar{\varepsilon}, \bar{R}}$ tends to zero uniformly with $\bar{\varepsilon}$.

Step 3 : The interior Dirichlet to Neumann map. We have already proved the analogue of Proposition 7 in Proposition 5, so we may pass directly to the corresponding interior Dirichlet to Neumann map. As above, the unique solution of the homogeneous problem

$$
\left\{\begin{array}{rlll}
\mathcal{L}_{\bar{\varepsilon}, \bar{R}} w & =0 & \text { in } \quad B\left(x_{i}, 1\right) \\
w & =\psi_{i} & \text { on } \quad \partial B\left(x_{i}, 1\right)
\end{array}\right.
$$

with

$$
w=w_{\psi_{i}} \in \mathcal{C}_{\nu}^{2, \alpha}\left(B\left(x_{i}, 1\right) \backslash\left\{x_{i}\right\}\right) \oplus_{j=0}^{N} \operatorname{Span}\left\{\frac{1}{\varepsilon_{i}} \Psi_{\varepsilon_{i}}^{j,+}\left(\left(\cdot-x_{i}\right) / R_{i}\right)\right\}
$$

defines a continuous map

$$
\begin{aligned}
\Psi \in \oplus_{i=1}^{n} \mathcal{C}^{2, \alpha}\left(\partial B\left(x_{i}, 1\right)\right) \longrightarrow\left(w_{\psi_{1}}, \ldots, w_{\psi_{n}}\right) \in & \oplus_{i=1}^{n}\left(\mathcal{C}_{\nu}^{2, \alpha}\left(B\left(x_{i}, 1\right) \backslash\left\{x_{i}\right\}\right)\right. \\
& \left.\oplus_{j=0}^{N} \operatorname{Span}\left\{\frac{1}{\varepsilon_{i}} \Psi_{\varepsilon_{i}}^{j,+}\left(\left(\cdot-x_{i}\right) / R_{i}\right)\right\}\right) .
\end{aligned}
$$

Now define the map $\mathcal{T}_{\bar{\varepsilon}, \bar{R}}$ by

$$
\mathcal{T}_{\bar{\varepsilon}, \bar{R}}(\Psi)=\left(\mathcal{T}_{\bar{\varepsilon}, \bar{R}}^{1}\left(\psi_{1}\right), \ldots, \mathcal{T}_{\bar{\varepsilon}, \bar{R}}^{n}\left(\psi_{n}\right)\right)=\left(\left.\partial_{r_{1}} w_{\psi_{1}}\right|_{r_{1}=1}, \ldots,\left.\partial_{r_{n}} w_{\psi_{n}}\right|_{r_{n}=1}\right) \in \oplus_{i=1}^{n} \mathcal{C}^{1, \alpha}\left(\partial B\left(x_{i}, 1\right)\right) .
$$


Lemma 7 The norms of the mappings

$$
\mathcal{C}^{2, \alpha}\left(\partial B\left(x_{i}, 1\right)\right) \ni \psi_{i} \longrightarrow w_{\psi_{i}} \in \mathcal{C}_{\nu}^{2, \alpha}\left(B\left(x_{i}, 1\right) \backslash\left\{x_{i}\right\}\right) \oplus_{j=0}^{N} \operatorname{Span}\left\{\frac{1}{\varepsilon_{i}} \Psi_{\varepsilon_{i}}^{j,+}\left(\left(\cdot-x_{i}\right) / R_{i}\right)\right\}
$$

and

$$
\mathcal{T}_{\bar{\varepsilon}, \bar{R}}: \oplus_{i=1}^{n} \mathcal{C}^{2, \alpha}\left(\partial B\left(x_{i}, 1\right)\right) \longrightarrow \oplus_{i=1}^{n} \mathcal{C}^{1, \alpha}\left(\partial B\left(x_{i}, 1\right)\right)
$$

are bounded independently of $\varepsilon_{i}$ provided all $\varepsilon_{i}<\varepsilon_{0}$ and $\varepsilon_{0}$ is sufficiently small. The mappings $\mathcal{T}_{\bar{\varepsilon}, \bar{R}}$, defined from $\oplus_{i=1}^{n} H^{1}\left(\partial B\left(x_{i}, 1\right)\right)$ into $\oplus_{i=1}^{n} L^{2}\left(\partial B\left(x_{i}, 1\right)\right)$, converge in norm, as $\bar{\varepsilon}$ tends to 0 , to a limiting operator $\mathcal{T}_{0}$ which acts diagonally on n-tuples. In terms of the eigenfunctions $\phi_{j}$ for the Laplacian on $\partial B\left(x_{i}, 1\right)$, the $i^{\text {th }}$ component $\mathcal{T}_{0}^{i}$ is determined by

$$
\begin{gathered}
\mathcal{T}_{0}^{i}\left(\phi_{j}\right)=\left(\frac{2-N}{2}+\left(\frac{(N-2)^{2}}{4}+\lambda_{j}\right)^{1 / 2}\right) \phi_{j} \text { for all } j \geq 1, \\
\mathcal{T}_{0}^{i}\left(\phi_{0}\right)=-\frac{N-2}{2}\left(1+\frac{\cosh \left((N-2) \log R_{i} / 2\right)}{\sinh \left((N-2) \log R_{i} / 2\right)}\right) \phi_{0}=(2-N)\left(\frac{R_{i}^{N-2}}{R_{i}^{N-2}-1}\right) \phi_{0} .
\end{gathered}
$$

Proof : We only need demonstrate the convergence of the $\mathcal{T}_{\bar{\varepsilon}, \bar{R}}$ and the specific form of the limit $\mathcal{T}_{0}$. This will follow from the proof of Lemma 2 .

By Lemma 2, for each $j \geq N+1$, there exists a unique solution $y_{j}(t)$ of the equation

$$
\frac{d^{2} y}{d t^{2}}-\left(\frac{(N-2)^{2}}{4}+\lambda_{j}-\frac{N(N+2)}{4} v_{\varepsilon_{i}, R_{i}}^{\frac{4}{N-2}}\right) y=0
$$

which satisfies $y_{j}(0)=1$ and which decays exponentially as $t \rightarrow+\infty$. In this equation $v_{\varepsilon_{i}, R_{i}}=$ $v_{\varepsilon_{i}}\left(t+\log R_{i}\right)$. Let $\gamma_{j}=\left(\frac{(N-2)^{2}}{4}+\lambda_{j}\right)^{1 / 2}$ and $\delta_{j}=\left(\gamma_{j}^{2}-\frac{N(N+2)}{4}\right)^{1 / 2}$. As in the proof of that Lemma, we show that

$$
\left|y_{j}(t)\right| \leq e^{-\delta_{j} t} \quad \text { for all } \quad t \geq 0 .
$$

Integrating the product of (42) with $e^{-\gamma_{j} t}$ from 0 to $+\infty$ and integrating by parts using that $y_{j}(0)=1$, we find that

$$
\frac{d y_{j}}{d t}(0)+\gamma_{j}=\frac{N(N+2)}{4} \int_{0}^{+\infty} v_{\varepsilon_{i}, R_{i}}^{\frac{4}{N-2}} y_{j}(t) e^{-\gamma_{j} t} d t .
$$

Since $v_{\varepsilon_{i}, R_{i}}(t) \leq R_{i}^{\frac{N-2}{2}} \varepsilon_{i} e^{\frac{N-2}{2} t}$ and also since $\left|v_{\varepsilon_{i}, R_{i}}(t)\right| \leq 1$ for all $t$, we obtain

$$
\left|\frac{d y_{j}}{d t}(0)+\gamma_{j}\right| \leq \frac{N(N+2)}{4} \int_{0}^{T_{\varepsilon} / 2}\left(\varepsilon_{i} R_{i}^{\frac{N-2}{2}} e^{\frac{N-2}{2} t}\right)^{\frac{4}{N-2}} e^{-\left(\delta_{j}+\gamma_{j}\right) t} d t+\int_{T_{\varepsilon} / 2}^{+\infty} e^{-\left(\delta_{j}+\gamma_{j}\right) t} d t .
$$

Since $T_{\varepsilon} \sim-\frac{4}{N-2} \log \varepsilon$, this expression can be bounded by

$$
\left|\frac{d y_{j}}{d t}(0)+\gamma_{j}\right| \leq C_{N, R_{i}} \varepsilon^{\frac{4}{N-2}}\left(\gamma_{j}+\delta_{j}\right)^{-1},
$$

for some constant $C_{N, R_{i}}>0$. In particular, we see that, for fixed $j \geq N+1$,

$$
\lim _{\varepsilon_{i} \rightarrow 0} \frac{d y_{j}}{d t}(0)=-\gamma_{j}
$$

Now, the solution of (41) with $\psi_{i}=\phi_{j}$ is given by $w(x)=\left|x-x_{i}\right|^{\frac{2-N}{2}} y_{j}\left(-\log \left|x-x_{i}\right|\right) \phi_{j}(\theta)$, and its Neumann data is $\left(\frac{2-N}{2}-\frac{d y_{j}}{d t}(0)\right) \phi_{j}(\theta)$. The relevant solution when $\varepsilon_{i}=0$ is $\mid x-$ $\left.x_{i}\right|^{\frac{2-N}{2}+\gamma_{j}} \phi_{j}(\theta)$. 
Hence if $\psi(\theta)=\sum_{j=N+1}^{+\infty} a_{j} \phi_{j}(\theta)$, we see that

$$
\begin{aligned}
\left\|\mathcal{T}_{\bar{\varepsilon}, \bar{R}}^{i}(\psi)-\mathcal{T}_{0}^{i}(\psi)\right\|_{L^{2}}^{2} & =\sum_{j=N+1}^{+\infty}\left|a_{j}\right|^{2}\left(\left(\frac{2-N}{2}-\frac{d y_{j}}{d t}(0)\right)-\left(\frac{2-N}{2}+\gamma_{j}\right)\right)^{2} \\
& \leq C \varepsilon^{\frac{4}{N-2}} \sum_{j=N+1}^{+\infty}\left|a_{j}\right|^{2} \\
& \leq C \varepsilon^{\frac{4}{N-2}}\|\Psi\|_{L^{2}}^{2} \leq C \varepsilon^{\frac{4}{N-2}}\|\Psi\|_{H^{1}}^{2},
\end{aligned}
$$

and this expression tends to 0 with $\varepsilon_{i}$.

When $j=0, \ldots, N$, we can proceed by direct computation. Indeed, when $j=1, \ldots, N$ and $\psi_{i}$ is the eigenfunction $\phi_{j}$, then

$$
\left|x-x_{i}\right|^{\frac{4-N}{2}}\left(\Phi_{\varepsilon_{i}}^{1,+}\left(\log R_{i}\right)\right)^{-1} \Phi_{\varepsilon_{i}}^{1,+}\left(-\log \left|x-x_{i}\right|+\log R_{i}\right) \phi_{j}(\theta)
$$

is the solution of (41). An explicit computation, using Proposition 3, shows that

$$
\lim _{\bar{\varepsilon} \rightarrow 0} \mathcal{T}_{\bar{\varepsilon}, \bar{R}}^{i}\left(\phi_{j}\right)=\phi_{j}
$$

as expected. Similarly, when $j=0$, the explicit solution of (41) is given by

$$
\left|x-x_{i}\right|^{\frac{2-N}{2}}\left(\Psi_{\varepsilon_{i}}^{0,+}\left(\log R_{i}\right)\right)^{-1} \Psi_{\varepsilon_{i}}^{0,+}\left(-\log \left|x-x_{i}\right|+\log R_{i}\right) .
$$

Using Proposition 3 again, we get

$$
\lim _{\bar{\varepsilon} \rightarrow 0} \mathcal{T}_{\bar{\varepsilon}, \bar{R}}^{i}\left(\phi_{0}\right)=-\frac{N-2}{2}\left(1+\frac{\cosh \left((N-2) \log R_{i} / 2\right)}{\sinh \left((N-2) \log R_{i} / 2\right)}\right) .
$$

Step 4: Invertibility of the difference of Dirichlet to Neumann maps. In order to glue the interior and exterior solutions together, we must add correction terms to these solutions. These correction terms are solutions of the homogeneous problem, and are chosen so that the Cauchy data from the inside and outside match up. To find these, we must show that the difference $\mathcal{S}_{\bar{\varepsilon}, \bar{R}}-\mathcal{T}_{\bar{\varepsilon}, \bar{R}}$ is invertible when all $\varepsilon_{i}$ are sufficiently small.

Proposition 8 There exists $\varepsilon_{0}>0$ such that for $\varepsilon<\varepsilon_{0}$,

$$
\mathcal{S}_{\bar{\varepsilon}, \bar{R}}-\mathcal{T}_{\bar{\varepsilon}, \bar{R}}: \oplus_{i=1}^{n} \mathcal{C}^{2, \alpha}\left(\partial B\left(x_{i}, 1\right)\right) \longrightarrow \oplus_{i=1}^{n} \mathcal{C}^{1, \alpha}\left(\partial B\left(x_{i}, 1\right)\right)
$$

is invertible. The norm of its inverse is bounded by some constant $C>0$ independent of $\varepsilon$.

Proof : By the $L^{2}$ operator norm convergence of $\mathcal{S}_{\bar{\varepsilon}, \bar{R}}-\mathcal{T}_{\bar{\varepsilon}, \bar{R}}$ to $\mathcal{S}_{0}-\mathcal{T}_{0}$, it suffices to show that $\mathcal{S}_{0}-\mathcal{T}_{0}$ is invertible. Now $\mathcal{S}_{0}-\mathcal{T}_{0}$ is a self-adjoint first order pseudodifferential operator. Both $\mathcal{S}_{0}$ and $\mathcal{T}_{0}$ are elliptic, with principal symbols $|\xi|$ and $-|\xi|$, respectively, hence the difference is also elliptic and semibounded. This means that $\mathcal{S}_{0}-\mathcal{T}_{0}$ has discrete spectrum, and thus we need only prove that it is injective. The invertibility in Hölder spaces then follows by standard regularity theory.

We argue by contradiction. Assume that $\mathcal{S}_{0}-\mathcal{T}_{0}$ is not injective. Then there exists some $\Psi_{0} \in \oplus_{i=1}^{n} \mathcal{C}^{\infty}\left(\partial B\left(x_{i}, 1\right)\right)$ for which $\left(\mathcal{S}_{0}-\mathcal{T}_{0}\right)\left(\Psi_{0}\right)=0$. We may extend the Dirichlet data $\Psi_{0}$ to a harmonic function on the exterior region, decaying at infinity, and also one on the interior region. $\mathcal{S}_{0}$ is the Dirichlet to Neumann map for the ordinary Laplacian on the exterior region, while $\mathcal{T}_{0}$ is the Dirichlet to Neumann map for the Laplacian on the union of balls only for the spherical eigencomponents with index $j \geq 1$. Thus $\Psi_{0}$ extends to harmonic functions $w^{\prime}$ and $w^{\prime \prime}$ on the interior and exterior regions, respectively. These functions have the same Dirichlet data, namely 
$\Psi_{0}$, and by assumption on this Dirichlet data, all eigencomponents with $j \geq 1$ of their Neumann data agree as well. To find a harmonic function with the $j=0$ eigencomponent of the Neumann data also matching at these spheres, it suffices to add on appropriate multiples of the functions $\left|x-x_{i}\right|^{2-N}-1$ on each $B\left(x_{i}, 1\right)$. We obtain in this way a harmonic function $w$ on $\mathbb{R}^{N} \backslash \Sigma$ which decays at infinity, and with at worst a radial singular term at each $x_{i}$. The only possibility is that this function has the form

$$
w(x)=\sum_{i=1}^{n} p_{i}\left|x-x_{i}\right|^{2-N} .
$$

We now find the radial part, $w_{0}^{i}(x)$, of the harmonic function $w(x)$ in each ball $B\left(x_{i}, 1\right)$, and use this to compute $T_{0}^{i} \Psi_{0}$ directly. Comparing this with the previous answer will lead to a contradiction. In $B\left(x_{i_{0}}, 1\right)$, this radial part is a sum of the singular part and a constant:

$$
w_{0}^{i_{0}}(x)=p_{i_{0}} r_{i_{0}}^{2-N}+\sum_{i \neq i_{0}} \frac{1}{\omega_{N-1}} \int_{S^{N-1}} p_{i}\left|r_{i_{0}} \theta+x_{i_{0}}-x_{i}\right|^{2-N} d \theta,
$$

where $r_{i_{0}}=\left|x-x_{i_{0}}\right|$. Here $\omega_{N-1}$ is the volume of $S^{N-1}$. The terms in the sum may be computed by noticing that the function

$$
x \rightarrow \sum_{i \neq i_{0}} p_{i}\left|x-x_{i}\right|^{2-N}
$$

is harmonic in $B\left(x_{i_{0}}, 2\right)$, hence, by the mean value property, its value at $x_{i_{0}}$ is equal to its average over the unit sphere centerd at $x_{i_{0}}$. We conclude that

$$
w_{0}^{i_{0}}(x)=p_{i_{0}}\left|x-x_{i_{0}}\right|^{2-N}+\sum_{i \neq i_{0}} p_{i}\left|x_{i}-x_{i_{0}}\right|^{2-N}
$$

The normal derivative of this function at $B\left(x_{i_{0}}, 1\right)$ is just $(2-N) p_{i_{0}}$, but on the other hand, it must agree with $T_{0}^{i_{0}}\left(w_{0}^{i_{0}}\right)$. This leads to the equality

$$
(2-N) p_{i_{0}}=(2-N) \frac{R_{i_{0}}^{N-2}}{R_{i_{0}}^{N-2}-1}\left(p_{i_{0}}+\sum_{i \neq i_{0}} p_{i}\left|x_{i}-x_{i_{0}}\right|^{2-N}\right)
$$

or simply

$$
p_{i_{0}}+R_{i_{0}}^{N-2} \sum_{i \neq i_{0}} p_{i}\left|x_{i}-x_{i_{0}}\right|^{2-N}=0
$$

Now recall that the parameters $R_{i}$ satisfy the relation (12),

$$
\sum_{i \neq i_{0}} R_{i}^{\frac{N-2}{2}} R_{i_{0}}^{\frac{N-2}{2}} q_{i}\left|x_{i_{0}}-x_{i}\right|^{2-N}=q_{i_{0}}
$$

A small calculation shows that the collection of numbers $\tilde{p}_{i} \equiv \frac{p_{i}}{q_{i}}\left(R_{i}\right)^{\frac{2-N}{2}}$ give a solution of the system

$$
\sum_{i \neq i_{0}} R_{i}^{\frac{N-2}{2}} R_{i_{0}}^{\frac{N-2}{2}} q_{i} q_{i_{0}}\left|x_{i_{0}}-x_{i}\right|^{2-N}\left(\tilde{p}_{i}+\tilde{p}_{i_{0}}\right)=0, \quad i_{0}=1, \ldots, n .
$$

Let $s_{i, j}=R_{i}^{\frac{N-2}{2}} R_{j}^{\frac{N-2}{2}} q_{i} q_{j}\left|x_{j}-x_{i}\right|^{2-N}$. Multiply (44) by $\tilde{p}_{i_{0}}$ and sum over $i_{0}$ to obtain

$$
\sum_{i_{0}=1}^{n}\left(\sum_{i \neq i_{0}} s_{i, i_{0}}\left(\tilde{p}_{i}+\tilde{p}_{i_{0}}\right)\right) \tilde{p}_{i_{0}}=\sum_{i<i_{0}} s_{i, i_{0}}\left(\tilde{p}_{i}+\tilde{p}_{i_{0}}\right)^{2}=0 .
$$


Since all the $s_{i, i_{0}}$ are positive, we get that $\tilde{p}_{i}=-\tilde{p}_{i_{0}}$. But since $n \geq 3$, this forces the vanishing of all $\tilde{p}_{i}$. This is a contradiction, and the proof is finished.

Step 5: The correction term. Let $f \in \mathcal{C}_{\nu-2,-2}^{0, \alpha}\left(\mathbb{R}^{N} \backslash \Sigma\right)$. By Proposition 5 and Proposition 7 , there exist functions $w_{\text {ext }}$ and $w_{i n, i}, i=1, \ldots, n$, such that

$$
\left\{\begin{aligned}
\mathcal{L}_{\bar{\varepsilon}, \bar{R}} w_{\text {ext }}=f & \text { in } \Omega \\
w_{\text {ext }} & =0 \quad \text { on } \partial \Omega
\end{aligned}\right.
$$

and, for $i=1, \ldots, n$,

$$
\left\{\begin{aligned}
\mathcal{L}_{\bar{\varepsilon}, \bar{R}} w_{i n t, i} & =f \text { in } B\left(x_{i}, 1\right) \\
w_{\text {int }, i} & =0 \text { on } \partial B\left(x_{i}, 1\right)
\end{aligned}\right.
$$

From Proposition 5, there is a decomposition inside each $B\left(x_{i}, 1\right)$

$$
w_{i n t, i}(x)=G_{\varepsilon_{i}, R_{i}}\left(f_{i}\right)(x)+\sum_{j=0}^{n} K_{\varepsilon_{i}, R_{i}}^{j}\left(f_{i}\right)(x) \frac{1}{\varepsilon_{i}} \Psi_{\varepsilon_{i}}^{j,+}\left(\frac{x-x_{i}}{R_{i}}\right),
$$

where $f_{i}=f 1_{B\left(x_{i}, 1\right)}$. In addition, for some $c>0$

$$
\left\|G_{\varepsilon_{i}, R_{i}}\left(f_{i}\right)\right\|_{2, \alpha, \nu}+\sum_{j=0}^{N}\left|K_{\varepsilon_{i}, R_{i}}^{j}\left(f_{i}\right)\right| \leq c\|f\|_{0, \alpha, \nu-2,-2}
$$

and

$$
\left\|w_{\text {ext }}\right\|_{2, \alpha, \nu, 2} \leq c\|f\|_{0, \alpha, \nu-2,-2} \text {. }
$$

We now seek a function $w_{k e r}$ defined in $\mathbb{R}^{N} \backslash\left\{\cup_{i=1}^{n} \partial B\left(x_{i}, 1\right) \cup \Sigma\right\}$ so that

$$
w(x) \equiv\left\{\begin{aligned}
w_{\text {ext }}(x)+w_{\text {ker }}(x) & \text { in } \Omega \\
w_{\text {int }, i}(x)+w_{\text {ker }}(x) & \text { in } B\left(x_{i}, 1\right), \quad i=1, \ldots, n,
\end{aligned}\right.
$$

will satisfy $\mathcal{L}_{\bar{\varepsilon}, \bar{R}} w=f$ on $\mathbb{R}^{N} \backslash \Sigma$.

For this to be true we need to choose $w_{k e r}$ to be continuous across each $\partial B\left(x_{i}, 1\right)$, to satisfy $\mathcal{L}_{\bar{\varepsilon}, \bar{R}} w_{\text {ker }}=0$ away from these boundaries (and from $\Sigma$ ), and such that the jump of $\partial_{r_{i}} w_{k e r}$ across $\partial B\left(x_{i}, 1\right)$ equals $\partial_{r_{i}} w_{i n t, i}-\partial_{r_{i}} w_{e x t}$. This is equivalent to finding a solution $\Psi$ of

$$
\left(\mathcal{S}_{\bar{\varepsilon}, \bar{R}}-\mathcal{T}_{\bar{\varepsilon}, \bar{R}}\right)(\Psi)=\left\{\left(\partial_{r_{1}} w_{i n t, 1}-\partial_{r_{1}} w_{\text {ext }}\right), \ldots,\left(\partial_{r_{k}} w_{i n t, k}-\partial_{r_{k}} w_{\text {ext }}\right)\right\} \in \oplus_{i=1}^{n} \mathcal{C}^{1, \alpha}\left(\partial B\left(x_{i}, 1\right)\right) .
$$

This is possible by Proposition 8; this same result gives the uniform bound on the norm of $\Psi$. All estimates for the norm of $w$ follow from Lemma 6 and Lemma 7.

\section{The true linearization}

We now undertake the main task of analyzing the linearization about $\bar{u}_{\bar{\varepsilon}}(\bar{R}, \bar{a}, x)$. Recall that our ultimate goal is to find a solution of the nonlinear equation

$$
\mathcal{N}(w) \equiv \Delta\left(\bar{u}_{\bar{\varepsilon}}(\bar{R}, \bar{a}, \cdot)+w\right)+\frac{N(N-2)}{4}\left(\bar{u}_{\bar{\varepsilon}}(\bar{R}, \bar{a}, \cdot)+w\right)^{\frac{N+2}{N-2}}=0,
$$

for some choice of parameters $\bar{a}, \bar{R}$ (recall that $\bar{\varepsilon}$ is regarded as fixed), and for some $w$ which is dominated by $\bar{u}_{\bar{\varepsilon}}(\bar{R}, \bar{a}, x)$ near $\Sigma$. Let

$$
\Lambda_{\bar{\varepsilon}, \bar{R}, \bar{a}}=\Delta+\frac{N(N+2)}{4}\left(\bar{u}_{\bar{\varepsilon}}(\bar{R}, \bar{a}, \cdot)\right)^{\frac{4}{N-2}}
$$


denote the linearization of this equation about $w=0$. As is usual for a Jacobi operator, one can obtain solutions of $\Lambda_{\bar{\varepsilon}, \bar{R}, \bar{a}} w=0$ as derivatives of families of solutions of (45). Of course, we do not know any families of solutions yet, but we can obtain approximate solutions of $\Lambda_{\bar{\varepsilon}, \bar{R}, \bar{a}} w=0$ from families of approximate solutions of (45), and we know many explicit families of approximate solutions. In particular, the functions

$$
\Psi_{\bar{\varepsilon}, \bar{R}, \bar{a}, i}^{0,+}(x)=\partial_{R_{i}} \bar{u}_{\bar{\varepsilon}}(\bar{R}, \bar{a}, x), \quad \text { and } \quad \Psi_{\bar{\varepsilon}, \bar{R}, \bar{a}, i}^{j,+}(x)=\partial_{a_{i}^{j}} \bar{u}_{\bar{\varepsilon}}(\bar{R}, \bar{a}, x), \quad j=1, \ldots, N, \quad i=1, \ldots, n,
$$

defined by differentiating $\bar{u}_{\bar{\varepsilon}}(\bar{R}, \bar{a}, x)$ with respect to $R_{i}$ and $a_{i}^{j}$, respectively, have the property that

$$
\Lambda_{\bar{\varepsilon}, \bar{R}, \bar{a}} \Psi_{\bar{\varepsilon}, \bar{R}, \bar{a}, i}^{j,+}(x)
$$

vanishes near each $x_{i}$. This may be seen by differentiating (45) with respect to either $R_{i}$ or $a_{i}^{j}$.

In this section we shall consider the problem $\Lambda_{\bar{\varepsilon}, \bar{R}, \bar{a}} w=f$, for $f \in \mathcal{C}_{\nu-2,-2}^{0, \alpha}\left(\mathbb{R}^{N} \backslash \Sigma\right)$. The main linear result in this paper is that we can find a solution with optimal bounds in the space

$$
\mathcal{M}(\bar{a})=\mathcal{C}_{\nu, 2}^{2, \alpha}\left(\mathbb{R}^{N} \backslash \Sigma\right) \oplus \operatorname{Span}\left\{\Psi_{\bar{\varepsilon}, \bar{R}, \bar{a}, i}^{j,+}\right\}
$$

where the norm of an element $w(x)=v(x)+\sum_{i} S_{i} \Psi_{\bar{\varepsilon}, \bar{R}, \bar{a}, i}^{0,+}+\sum_{i} \sum_{j=1}^{N} \alpha_{i}^{j} \Psi_{\bar{\varepsilon}, \bar{R}, \bar{a}, i}^{j,+}$ is given by

$$
\|w\|_{\mathcal{M}(\bar{a})}=\rho^{\nu}\|v\|_{2, \alpha, \nu, 2}+\varepsilon \sum_{i}\left|S_{i}\right|+\varepsilon \rho \sum_{i, j}\left|\alpha_{i}^{j}\right| .
$$

As before, the deficiency space spanned by the $\Psi_{\bar{\varepsilon}, \bar{R}, \bar{a}, i}^{j,+}$ is necessary to obtain surjectivity of the linearized operator. The rather byzantine choice of norms on $\mathcal{M}(\bar{a})$ is necessitated by the rather intricate estimates below, and the need for uniformity as $\varepsilon$ tends to zero. The following proposition states the expected bijectivity of $\Lambda_{\bar{\varepsilon}, \bar{R}, \bar{a}}$, but it also gives a rather sharper estimate on the solution in terms of the linear functionals $\mathcal{K}_{\bar{\varepsilon}, \bar{R}, i}^{j}$ introduced in Proposition 6 . The values $\mathcal{K}_{\bar{\varepsilon}, \bar{R}, i}^{j}(f)$ are closely related to the coefficients $S_{i}$ and $\alpha_{i}^{j}$ for the solution $w \in \mathcal{M}(\bar{a})$ of $\Lambda_{\bar{\varepsilon}, \bar{R}, \bar{a}} w=f$, but they correspond to coefficients of the simpler, radial solutions $\Psi_{\varepsilon_{i}}^{j,+}$. It may seem rather unnatural to continue to use these radial solutions here, but once again, these sharper estimates seem necessary later.

For notational convenience, we shall often denote these approximate solutions by

$$
\Psi_{\bar{\varepsilon}, \bar{R}, \bar{a}, i}^{0,+}(x)=\mu_{i}(\bar{\varepsilon}, \bar{R}, \bar{a}, x), \quad \Psi_{\bar{\varepsilon}, \bar{R}, \bar{a}, i}^{j,+}(x)=\gamma_{i}^{j}(\bar{\varepsilon}, \bar{R}, \bar{a}, x)
$$

and we shall identify $w(x)=v(x)+\sum S_{i} \mu_{i}(\bar{\varepsilon}, \bar{R}, \bar{a}, x)+\sum \alpha_{i}^{j} \gamma_{i}^{j}(\bar{\varepsilon}, \bar{R}, \bar{a}, x)$ with $(v, \bar{S}, \bar{\alpha})$.

Proposition 9 Let $\bar{\varepsilon}$ be an admissible set of Delaunay parameters, and suppose that $R_{i}>1$ and $a_{i} \in \mathbb{R}^{N}$ are a collection of numbers and vectors satisfying (12) and (13). Then

$$
\Lambda_{\bar{\varepsilon}, \bar{R}, \bar{a}}: \mathcal{M}(\bar{a}) \longrightarrow \mathcal{C}_{\nu-2,-2}^{0, \alpha}\left(\mathbb{R}^{N} \backslash \Sigma\right)
$$

is surjective, provided all $\varepsilon_{i}$ are less than some sufficiently small number $\varepsilon_{0}$. Furthermore, for $f \in \mathcal{C}_{\nu-2,-2}^{0, \alpha}\left(\mathbb{R}^{N} \backslash \Sigma\right)$, there exists $w=(v, \bar{S}, \bar{\alpha})$, corresponding to

$$
w(x)=v(x)+\sum_{i=1}^{N} S_{i} \mu_{i}(\bar{\varepsilon}, \bar{R}, \bar{a}, x)+\sum_{i=1}^{n} \sum_{j=1}^{N} \alpha_{i}^{j} \gamma_{i}^{j}(\bar{\varepsilon}, \bar{R}, \bar{a}, x)
$$

solution of the equation $\Lambda_{\bar{\varepsilon}, \bar{R}, \bar{a}} w=f$, which satisfies

$$
\rho^{\nu}\|v\|_{2, \alpha, \nu, 2} \leq c\left(\rho^{\nu}|| v_{0} \|_{2, \alpha, \nu, 2}+\varepsilon\left|\bar{S}_{0}\right|+\rho \varepsilon\left|\bar{\alpha}_{0}\right|\right),
$$




$$
\varepsilon|\bar{S}| \leq c\left(\left(\rho^{\nu+1}+\rho^{\frac{N+2}{2}}\right) \|\left. v_{0}\right|_{2, \alpha, \nu, 2}+\varepsilon\left|\bar{S}_{0}\right|+\left(\rho^{2}+\rho^{\frac{N+4}{2}-\nu}\right) \varepsilon\left|\bar{\alpha}_{0}\right|\right)
$$

and

$$
\varepsilon \rho|\bar{\alpha}| \leq c\left(\left(\rho^{\nu+1}+\rho^{\frac{N+4}{2}}\right)\left\|v_{0}\right\|_{2, \alpha, \nu, 2}+\left(\rho+\rho^{\frac{N+2}{2}-\nu}\right)\left|\bar{S}_{0}\right|+\rho \varepsilon\left|\bar{\alpha}_{0}\right|\right),
$$

where $v_{0}=\mathcal{G}_{\bar{\varepsilon}, \bar{R}} f, S_{0, i}=\frac{1}{\varepsilon_{i}} \mathcal{K}_{\bar{\varepsilon}, \bar{R}, i}^{0}(f)$ and $\alpha_{0, i}^{j}=\frac{1}{\varepsilon_{i}} \mathcal{K}_{\bar{\varepsilon}, \bar{R}, i}^{j}(f)$.

Proof : Define the space

$$
\mathcal{M}=\mathcal{C}_{\nu, 2}^{2, \alpha}\left(\mathbb{R}^{N} \backslash \Sigma\right) \oplus \operatorname{Span}\left\{\tilde{\chi}\left(x-x_{i}\right) \tilde{\mu}_{i}, \tilde{\chi}\left(x-x_{i}\right) \tilde{\gamma}_{i}^{j}\right\},
$$

where now $\tilde{\chi}$ is the cutoff equalling one in each $B\left(x_{i}, 1\right)$ and vanishing outside the union of the $B\left(x_{i}, 2\right)$ and

$$
\tilde{\mu}_{i}\left(\varepsilon_{i}, R_{i}, x\right)=\partial_{R_{i}} u_{\varepsilon_{i}}\left(R_{i}, 0, x-x_{i}\right), \quad \tilde{\gamma}_{i}^{j}\left(\varepsilon_{i}, R_{i}, x\right)=\partial_{a_{i}^{j}} u_{\varepsilon_{i}}\left(R_{i}, 0, x-x_{i}\right) .
$$

An element $w=v+\sum S_{i} \tilde{\chi}\left(x-x_{i}\right) \tilde{\mu}_{i}+\sum \alpha_{i}^{j} \tilde{\chi}\left(x-x_{i}\right) \tilde{\gamma}_{i}^{j}$ has the norm

$$
\|w\|_{\mathcal{M}} \equiv\|(v, \bar{S}, \bar{\alpha})\|_{\mathcal{M}}=\|v\|_{2, \alpha, \nu, 2}+\varepsilon \sum_{i}\left|S_{i}\right|+\varepsilon \sum_{i, j}\left|\alpha_{i}^{j}\right|
$$

Proposition 6 may then be rephrased as the assertion that

$$
\mathcal{L}_{\bar{\varepsilon}, \bar{R}}: \mathcal{M} \longrightarrow \mathcal{C}_{\nu-2,-2}^{0, \alpha}\left(\mathbb{R}^{N} \backslash \Sigma\right)
$$

is an isomorphism, and that the inverse is bounded independently of $\bar{\varepsilon}$.

We shall prove this proposition by reducing it to Proposition 6 by a sequence of perturbations.

Step 1. We first claim that

$$
\Lambda_{\bar{\varepsilon}, \bar{R}, 0} \equiv \Delta+\frac{N(N+2)}{4}\left(\bar{u}_{\bar{\varepsilon}}(\bar{R}, 0, x)\right)^{\frac{4}{N-2}}: \mathcal{M} \longrightarrow \mathcal{C}_{\nu-2,-2}^{0, \alpha}\left(\mathbb{R}^{N} \backslash \Sigma\right)
$$

is also an isomorphism, with inverse bounded independently of $\bar{\varepsilon}$. To see this, we estimate the norm of the difference $A=\Lambda_{\bar{\varepsilon}, \bar{R}, 0}-\mathcal{L}_{\bar{\varepsilon}, \bar{R}}$. First, the difference

$$
\left|\left(\bar{u}_{\bar{\varepsilon}}(\bar{R}, 0, x)\right)^{\frac{4}{N-2}}-\sum_{i} \tilde{\chi}\left(x-x_{i}\right)\left(u_{\varepsilon_{i}}\left(R_{i}, 0, x-x_{i}\right)\right)^{\frac{4}{N-2}}\right|
$$

vanishes in each $\left|x-x_{i}\right| \leq \rho_{i}$ and is estimated by $c \varepsilon^{\frac{4}{N-2}} \operatorname{dist}(x, \Sigma)^{-4}$ when $\operatorname{dist}(x, \Sigma) \geq 1$. We get the estimate

$$
\varepsilon^{\frac{4}{N-2}}\left|x-x_{i}\right|^{N-5}
$$

for this difference in the annulus $\rho_{i} \leq\left|x-x_{i}\right| \leq 1$ from Corollary 2 .

Finally, from Proposition 3 we derive the bounds

$$
\left|\tilde{\mu}_{i}\left(\varepsilon_{i}, R_{i}, x\right)\right| \leq C \varepsilon\left|x-x_{i}\right|^{2-N} \quad \text { and } \quad\left|\tilde{\gamma}_{i}^{j}\left(\varepsilon_{i}, R_{i}, x\right)\right| \leq C \varepsilon\left|x-x_{i}\right|,
$$

which hold in particular for all $x$ satisfying $\operatorname{dist}\left(x, x_{i}\right) \in\left[\rho_{i}, 2\right]$. Thus, for $w \in \mathcal{M}$, we get

$$
\begin{gathered}
\left\|\left(\Lambda_{\bar{\varepsilon}, \bar{R}, 0}-\mathcal{L}_{\bar{\varepsilon}, \bar{R}}\right) w\right\|_{0, \alpha, \nu-2,-2}=\left\|\left(\bar{u}_{\bar{\varepsilon}}(\bar{R}, 0, x)^{\frac{4}{N-2}}-\sum_{i=1}^{n} \tilde{\chi}\left(x-x_{i}\right) u_{\varepsilon_{i}}\left(R_{i}, 0, x-x_{i}\right)^{\frac{4}{N-2}}\right) w\right\|_{0, \alpha, \nu-2,-2} \\
\leq c \rho^{N+2}\left(\|v\|_{2, \alpha, \nu, 2}+\varepsilon \rho^{-\nu-1}|\bar{S}|+\varepsilon\left(1+\rho^{N-2-\nu}\right)|\bar{\alpha}|\right)
\end{gathered}
$$


By (10), we can estimate the right side by an arbitrarily small multiple $\eta$ of $\|w\|_{\mathcal{M}}$, provided $\varepsilon$ is sufficiently small, i.e. $\|A w\|_{0, \alpha, \nu-2,-2} \leq \eta\|w\|_{\mathcal{M}}$. Letting $\mathcal{L}_{\bar{\varepsilon}, \bar{R}}^{-1}$ denote the inverse of the map (49), then

$$
\Lambda_{\bar{\varepsilon}, \bar{R}, 0}^{-1} \equiv\left(\mathcal{L}_{\bar{\varepsilon}, \bar{R}}+A\right)^{-1}=\mathcal{L}_{\bar{\varepsilon}, \bar{R}}^{-1}\left(I+A \mathcal{L}_{\bar{\varepsilon}, \bar{R}}^{-1}\right)^{-1}
$$

is a right inverse of the map (50). Since $\mathcal{L}_{\bar{\varepsilon}, \bar{R}}$ is invertible, and since $A$ is small in operator norm, $\Lambda_{\bar{\varepsilon}, \bar{R}, 0}$ is also invertible, hence $(53)$ is the unique inverse. Since $A$ and $\mathcal{L}_{\bar{\varepsilon}, \bar{R}}^{-1}$ are bounded independently of $\bar{\varepsilon}$, so is $\Lambda_{\bar{\varepsilon}, \bar{R}, 0}^{-1}$.

We now obtain sharper estimates for the solution of $\Lambda_{\bar{\varepsilon}, \bar{R}, 0} w=f$.

Lemma 8 Suppose that $f$ is any function in $\mathcal{C}_{\nu-2,-2}^{0, \alpha}$, and let $w=\Lambda_{\bar{\varepsilon}, \bar{R}, 0}^{-1} f=(v, \bar{S}, \bar{\alpha})$ and $w_{0}=\mathcal{L}_{\bar{\varepsilon}, \bar{R}}^{-1} f=\left(v_{0}, \bar{S}_{0}, \bar{\alpha}_{0}\right)$. Then

$$
\begin{gathered}
\|v\|_{2, \alpha, \nu, 2} \leq C\left(|| v_{0} \|_{2, \alpha, \nu, 2}+\rho^{N+1-\nu} \varepsilon\left|\bar{S}_{0}\right|+\rho^{N+2}\left(1+\rho^{N-2-\nu}\right) \varepsilon\left|\bar{\alpha}_{0}\right|\right), \\
\varepsilon|\bar{S}| \leq C\left(\rho^{N+2}|| v_{0} \|_{2, \alpha, \nu, 2}+\varepsilon\left|\bar{S}_{0}\right|+\rho^{N+2}\left(1+\rho^{N-2-\nu}\right) \varepsilon\left|\bar{\alpha}_{0}\right|\right),
\end{gathered}
$$

and

$$
\varepsilon|\bar{\alpha}| \leq C\left(\left.\rho^{N+2}|| v_{0}\right|_{2, \alpha, \nu, 2}+\rho^{N+1-\nu} \varepsilon\left|\bar{S}_{0}\right|+\varepsilon\left|\bar{\alpha}_{0}\right|\right)
$$

Remark 7 We point out explicitly again that the coefficients $\bar{S}_{0}$ and $\bar{\alpha}_{0}$ here are the values of the functionals $\mathcal{K}_{\bar{\varepsilon}, \bar{R}, i}^{j}(f)$ introduced in Proposition 6, corresponding to the radial model operator $\mathcal{L}_{\bar{\varepsilon}, \bar{R}}$. Specifically,

$$
\varepsilon_{i}\left(S_{0}\right)_{i}=\mathcal{K}_{\bar{\varepsilon}, \bar{R}, i}^{0}(f), \quad \varepsilon_{i}\left(\alpha_{0}\right)_{i}^{j}=\mathcal{K}_{\bar{\varepsilon}, \bar{R}, i}^{j}(f) .
$$

Although it might seem more natural to view the coefficients $\bar{S}$ and $\bar{\alpha}$ coming from the solution of $\Lambda_{\bar{\varepsilon}, \bar{R}, 0} w=f$ (and later, $\Lambda_{\bar{\varepsilon}, \bar{R}, \bar{a}} w=f$ ) in terms of them, it turns out to be simpler in the long run to view these as primary.

Proof: Rewrite (53) as

$$
\Lambda_{\bar{\varepsilon}, \bar{R}, 0}^{-1}=\mathcal{L}_{\bar{\varepsilon}, \bar{R}}^{-1}\left(I+A \mathcal{L}_{\bar{\varepsilon}, \bar{R}}^{-1}\right)^{-1}\left(I+A \mathcal{L}_{\bar{\varepsilon}, \bar{R}}^{-1}-A \mathcal{L}_{\bar{\varepsilon}, \bar{R}}^{-1}\right)=\mathcal{L}_{\bar{\varepsilon}, \bar{R}}^{-1}\left(I-\left(I+A \mathcal{L}_{\bar{\varepsilon}, \bar{R}}^{-1}\right)^{-1} A \mathcal{L}_{\bar{\varepsilon}, \bar{R}}^{-1}\right) .
$$

Applying this to $f$ yields

$$
w=w_{0}-\mathcal{L}_{\bar{\varepsilon}, \bar{R}}^{-1}\left(I+A \mathcal{L}_{\bar{\varepsilon}, \bar{R}}^{-1}\right)^{-1} A w_{0} .
$$

The estimates of the lemma follow by considering the different components of the two sides of this equation, and using the boundedness of $\mathcal{L}_{\bar{\varepsilon}, \bar{R}}^{-1}\left(I+A \mathcal{L}_{\bar{\varepsilon}, \bar{R}}^{-1}\right)^{-1}$, and (52).

Step 2. We now perturb further. We claim that $\Lambda_{\bar{\varepsilon}, \bar{R}, 0}$ is invertible from $\mathcal{M}(0)$ to $\mathcal{C}_{\nu-2,-2}^{0, \alpha}$, with inverse uniformly bounded in $\bar{\varepsilon}$. In fact, because of the uniform invertibility of (50), it suffices to show that the inclusion

$$
\mathcal{M} \longrightarrow \mathcal{M}(0)
$$

is uniformly bounded. Equivalently, we must show that

$$
\|w\|_{\mathcal{M}(0)} \leq C\|w\|_{\mathcal{M}}
$$

for some $C$ independent of $\varepsilon$. To calculate the norm of $w$ in $\mathcal{M}_{0}$, we must write this function in the form $w=\bar{v}+\sum S_{i} \mu_{i}(\bar{\varepsilon}, \bar{R}, 0, \cdot)+\sum \alpha_{i}^{j} \gamma_{i}^{j}(\bar{\varepsilon}, \bar{R}, 0, \cdot)$. Since $w=v+\tilde{\chi}\left(\sum S_{i} \tilde{\mu}_{i}\left(\varepsilon_{i}, R_{i}, \cdot\right)+\right.$ $\left.\sum \alpha_{i}^{j} \tilde{\gamma}_{i}^{j}\left(\varepsilon_{i}, R_{i}, \cdot\right)\right)$ we get

$$
\bar{v}=v+\sum S_{i}\left(\tilde{\chi} \tilde{\mu}_{i}\left(\varepsilon_{i}, R_{i}, \cdot\right)-\mu_{i}(\bar{\varepsilon}, \bar{R}, 0, \cdot)\right)+\sum \alpha_{i}^{j}\left(\tilde{\chi} \tilde{\gamma}_{i}^{j}\left(\varepsilon_{i}, R_{i}, \cdot\right)-\gamma_{i}^{j}(\bar{\varepsilon}, \bar{R}, 0, \cdot)\right) .
$$


Now observe that

$\tilde{\mu}_{i}-\mu_{i}=\left(1-\chi_{i}\right) \partial_{R_{i}}\left(u_{\varepsilon_{i}}\left(R_{i}, 0, x-x_{i}\right)-\bar{w}_{\bar{\varepsilon}}\right), \quad \tilde{\gamma}_{i}^{j}-\gamma_{i}^{j}=\left(1-\chi_{i}\right) \partial_{a_{i}^{j}}\left(u_{\varepsilon_{i}}\left(R_{i}, 0, x-x_{i}\right)-\bar{w}_{\bar{\varepsilon}}\right)$

in each $B\left(x_{i}, 1\right)$. From (15)

$$
\left\|\tilde{\chi} \tilde{\mu}_{i}-\mu_{i}\right\|_{2, \alpha, \nu, 2} \leq C \rho^{-\nu} \varepsilon, \quad \text { and } \quad\left\|\tilde{\chi} \tilde{\gamma}_{i}^{j}-\gamma_{i}^{j}\right\|_{2, \alpha, \nu, 2} \leq C \rho^{1-\nu} \varepsilon .
$$

Hence

$$
\left\|\sum S_{i}\left(\tilde{\chi} \tilde{\mu}_{i}-\mu_{i}\right)\right\|_{2, \alpha, \nu, 2}+\|\left.\sum \alpha_{i}^{j}\left(\tilde{\chi} \tilde{\gamma}_{i}^{j}-\gamma_{i}^{j}\right)\right|_{2, \alpha, \nu, 2} \leq C \rho^{-\nu}(\varepsilon|\bar{S}|+\varepsilon \rho|\bar{\alpha}|)
$$

and thus

$$
\|w\|_{\mathcal{M}(0)}=\rho^{\nu}|| \bar{v}\left\|_{2, \alpha, \nu, 2}+\varepsilon|\bar{S}|+\varepsilon \rho|\bar{\alpha}| \leq C\left(\|v\|_{2, \alpha, \nu, 2}+\varepsilon|\bar{S}|+\varepsilon \rho|\alpha|\right)=C|| w\right\|_{\mathcal{M}},
$$

as desired.

We also derive the precise estimates for the inverse of

$$
\Lambda_{\bar{\varepsilon}, \bar{R}, 0}: \mathcal{M}(0) \longrightarrow \mathcal{C}_{\nu-2,-2}^{0, \alpha}\left(\mathbb{R}^{N} \backslash \Sigma\right)
$$

from the ones stated at the end of Step 1. In fact, replace $v$ by $\bar{v}$ there and use (54) and the sharp estimates for $\varepsilon|\bar{S}|$ and $\varepsilon \rho|\bar{\alpha}|$ to get

$$
\begin{gathered}
\rho^{\nu}\|\bar{v}\|_{2, \alpha, \nu, 2} \leq c\left(\rho^{\nu} \| v_{0}||_{2, \alpha, \nu, 2}+\varepsilon\left|\bar{S}_{0}\right|+\rho \varepsilon\left|\bar{\alpha}_{0}\right|\right), \\
\varepsilon|\bar{S}| \leq c\left(\rho^{N+2}\left\|v_{0}\right\|_{2, \alpha, \nu, 2}+\varepsilon\left|\bar{S}_{0}\right|+\rho^{N+2}\left(1+\rho^{N-2-\nu}\right) \varepsilon\left|\bar{\alpha}_{0}\right|\right),
\end{gathered}
$$

and

$$
\varepsilon \rho|\bar{\alpha}| \leq c\left(\rho^{N+3}|| v_{0} \|_{2, \alpha, \nu, 2}+\rho^{N+2-\nu} \varepsilon\left|\bar{S}_{0}\right|+\rho \varepsilon\left|\bar{\alpha}_{0}\right|\right),
$$

where $\left(v_{0}, \bar{S}_{0}, \bar{\alpha}_{0}\right)=\mathcal{L}_{\bar{\varepsilon}, \bar{R}}^{-1}(f)$.

Step 3. We perform a final perturbation of (55) to show that

$$
\Lambda_{\bar{\varepsilon}, \bar{R}, \bar{a}}: \mathcal{M}(\bar{a}) \longrightarrow \mathcal{C}_{\nu-2,-2}^{0, \alpha}\left(\mathbb{R}^{N} \backslash \Sigma\right)
$$

is an isomorphism, with inverse uniformly bounded as $\bar{\varepsilon}$ tends to zero. In order to apply the same sort of perturbation argument, we would like $\Lambda_{\bar{\varepsilon}, \bar{R}, \bar{a}}$ and $\Lambda_{\bar{\varepsilon}, \bar{R}, 0}$ to be acting on the same space of functions. To this end, define

$$
\tilde{\mathcal{M}}=\left\{(v, \bar{S}, \bar{a}) \in \mathcal{C}_{\nu, 2}^{2, \alpha}\left(\mathbb{R}^{N} \backslash \Sigma\right) \oplus \mathbb{R}^{n} \oplus\left(\mathbb{R}^{N}\right)^{n}\right\},
$$

with norm given by

$$
\|(v, \bar{S}, \bar{\alpha})\|_{\tilde{\mathcal{M}}}=\rho^{\nu}|| v \|_{2, \alpha, \nu, 2}+\varepsilon|\bar{S}|+\rho \varepsilon|\bar{\alpha}| .
$$

Then $\tilde{\mathcal{M}}$ is equivalent to any of the spaces $\mathcal{M}(\bar{a})$ via the map

$$
\iota_{\bar{a}}:(v, \bar{S}, \bar{\alpha}) \longrightarrow v(x)+\sum S_{i} \mu_{i}(\bar{\varepsilon}, \bar{R}, \bar{a}, x)+\sum \alpha_{i}^{j} \gamma_{i}^{j}(\bar{\varepsilon}, \bar{R}, \bar{a}, x) .
$$

We denote

$$
\tilde{\Lambda}_{\bar{\varepsilon}, \bar{R}, \bar{a}}=\Lambda_{\bar{\varepsilon}, \bar{R}, \bar{a}} \circ \iota_{\bar{a}}: \tilde{\mathcal{M}} \longrightarrow \mathcal{C}_{\nu-2,-2}^{0, \alpha}\left(\mathbb{R}^{N} \backslash \Sigma\right) .
$$

The uniformly bounded inverse $\tilde{\Lambda}_{\bar{\varepsilon}, \bar{R}, 0}^{-1}$ is a first approximation to the inverse of $\tilde{\Lambda}_{\bar{\varepsilon}, \bar{R}, \bar{a}}$. In fact,

$$
\tilde{\Lambda}_{\bar{\varepsilon}, \bar{R}, 0}^{-1} \tilde{\Lambda}_{\bar{\varepsilon}, \bar{R}, \bar{a}}=I+\tilde{\Lambda}_{\bar{\varepsilon}, \bar{R}, 0}^{-1}\left(\tilde{\Lambda}_{\bar{\varepsilon}, \bar{R}, \bar{a}}-\tilde{\Lambda}_{\bar{\varepsilon}, \bar{R}, 0}\right) .
$$


We shall show that the final term $\tilde{\Lambda}_{\bar{\varepsilon}, \bar{R}, 0}^{-1}\left(\tilde{\Lambda}_{\bar{\varepsilon}, \bar{R}, \bar{a}}-\tilde{\Lambda}_{\bar{\varepsilon}, \bar{R}, 0}\right)$ has small norm as a map on $\tilde{\mathcal{M}}$. Because of the uniform boundedness of $\tilde{\Lambda}_{\bar{\varepsilon}, \bar{R}, 0}^{-1}$, it should suffice to show that the difference

$$
\tilde{\Lambda}_{\bar{\varepsilon}, \bar{R}, \bar{a}}-\tilde{\Lambda}_{\bar{\varepsilon}, \bar{R}, 0}: \tilde{\mathcal{M}} \longrightarrow \mathcal{C}_{\nu-2,-2}^{0, \alpha}\left(\mathbb{R}^{N} \backslash \Sigma\right)
$$

has small norm. This is almost true, although at one stage of the argument we require slightly more information about the inverse. However, let us concentrate on showing that most terms in this difference are small.

Let $w=(v, \bar{S}, \bar{a}) \in \tilde{\mathcal{M}}$, and for convenience write

$$
U_{\bar{a}}(x)=\sum S_{i} \mu_{i}(\bar{\varepsilon}, \bar{R}, \bar{a}, x)+\sum \alpha_{i}^{j} \gamma_{i}^{j}(\bar{\varepsilon}, \bar{R}, \bar{a}, x) .
$$

Then

$$
\left(\tilde{\Lambda}_{\bar{\varepsilon}, \bar{R}, \bar{a}}-\tilde{\Lambda}_{\bar{\varepsilon}, \bar{R}, 0}\right)(w)=\left(\bar{u}_{\bar{\varepsilon}}(\bar{R}, \bar{a}, x)^{\frac{4}{N-2}}-\bar{u}_{\bar{\varepsilon}}(\bar{R}, 0, x)^{\frac{4}{N-2}}\right) v+\left(\Lambda_{\bar{\varepsilon}, \bar{R}, \bar{a}} U_{\bar{a}}(x)-\Lambda_{\bar{\varepsilon}, \bar{R}, 0} U_{0}(x)\right) .
$$

We estimate the two terms on the right in turn.

The first is simpler. Going back to the definition of $\bar{u}_{\bar{\varepsilon}}(\bar{R}, \bar{a}, x)$, we expand in $B\left(x_{i}, \rho_{i}\right)$ to get

$$
\begin{gathered}
\left(\bar{u}_{\bar{\varepsilon}}(\bar{R}, \bar{a}, x)\right)^{\frac{4}{N-2}}-\left(\bar{u}_{\bar{\varepsilon}}(\bar{R}, 0, x)\right)^{\frac{4}{N-2}}= \\
2 a_{i} \cdot\left(x-x_{i}\right)\left|x-x_{i}\right|^{-2} v_{\varepsilon_{i}}^{\frac{6-N}{N-2}}\left(-\log \left|x-x_{i}\right|+\log R_{i}\right)\left(v_{\varepsilon_{i}}\left(-\log \left|x-x_{i}\right|+\log R_{i}\right)\right. \\
\left.-\frac{2}{N-2} \dot{v}_{\varepsilon_{i}}\left(-\log \left|x-x_{i}\right|+\log R_{i}\right)\right)+O(1) v_{\varepsilon_{i}}^{\frac{4}{N-2}}\left(-\log \left|x-x_{i}\right|+\log R_{i}\right) .
\end{gathered}
$$

Here we have used the fact that for all $T>0$, there exists some $c_{T}>0$, independent of $\varepsilon \leq \varepsilon_{0}$, such that for all $t_{0} \in[-T, T]$

$$
v_{\varepsilon}\left(t+t_{0}\right)+\left|\dot{v}_{\varepsilon}\left(t+t_{0}\right)\right|+\left|\ddot{v}_{\varepsilon}\left(t+t_{0}\right)\right| \leq c_{T} v_{\varepsilon}(t) .
$$

We consider this difference in three separate regions about each $x_{i}$. In the innermost one, where $\operatorname{dist}\left(x, x_{i}\right) \leq \rho^{\frac{N+2}{2}}=\varepsilon^{\frac{2}{N-2}}$, since $v_{\varepsilon}$ and $\dot{v}_{\varepsilon}$ are bounded we get

$$
\left|\left(\bar{u}_{\bar{\varepsilon}}(\bar{R}, \bar{a}, x)\right)^{\frac{4}{N-2}}-\left(\bar{u}_{\bar{\varepsilon}}(\bar{R}, 0, x)\right)^{\frac{4}{N-2}}\right| \leq C\left|x-x_{i}\right|^{-1} .
$$

For the middle region, where $\operatorname{dist}\left(x, x_{i}\right) \in\left[\varepsilon^{\frac{2}{N-2}}, \rho_{i}\right]$, we further expand (58), using Proposition 3 , to get

$$
\left|\left(\bar{u}_{\bar{\varepsilon}}(\bar{R}, \bar{a}, x)\right)^{\frac{4}{N-2}}-\left(\bar{u}_{\bar{\varepsilon}}(\bar{R}, 0, x)\right)^{\frac{4}{N-2}}\right| \leq C\left(\varepsilon^{\frac{4}{N-2}}\left|x-x_{i}\right|^{-2}+\varepsilon^{\frac{8}{N-2}}\left|x-x_{i}\right|^{-5}\right) .
$$

A similar estimate also holds if $\operatorname{dist}\left(x, x_{i}\right) \in\left[\rho_{i}, 2 \rho_{i}\right]$. Finally, when $\operatorname{dist}\left(x, x_{i}\right) \geq 2 \rho_{i}$ for all $i$, then

$$
\left(\bar{u}_{\bar{\varepsilon}}(\bar{R}, \bar{a}, x)\right)^{\frac{4}{N-2}}-\left(\bar{u}_{\bar{\varepsilon}}(\bar{R}, 0, x)\right)^{\frac{4}{N-2}}=0,
$$

since $\bar{u}_{\bar{\varepsilon}}$ does not depend on $\bar{a}$ if $\operatorname{dist}\left(x, x_{i}\right) \geq 2 \rho_{i}$. The estimates for the full Hölder norms are similar.

Putting these estimates together, we get

$$
\left\|\left(\bar{u}_{\bar{\varepsilon}}(\bar{R}, \bar{a}, x)^{\frac{4}{N-2}}-\bar{u}_{\bar{\varepsilon}}(\bar{R}, 0, x)^{\frac{4}{N-2}}\right) v\right\|_{0, \alpha, \nu-2,-2} \leq c \varepsilon^{\frac{2}{N-2}}\|v\|_{2, \alpha, \nu, 2} .
$$

The second term on the right in (57) is more complicated to estimate. First write

$$
\Lambda_{\bar{\varepsilon}, \bar{R}, \bar{a}}\left(U_{\bar{a}}\right)-\Lambda_{\bar{\varepsilon}, \bar{R}, 0}\left(U_{0}\right)=\Delta\left(U_{\bar{a}}-U_{0}\right)
$$




$$
+\frac{N(N+2)}{4}\left(\left(\bar{u}_{\bar{\varepsilon}}(\bar{R}, \bar{a}, x)\right)^{\frac{4}{N-2}} U_{\bar{a}}-\left(\bar{u}_{\bar{\varepsilon}}(\bar{R}, 0, x)\right)^{\frac{4}{N-2}} U_{0}\right) .
$$

Recall that $U_{\bar{a}}$ satisfies

$$
\Delta U_{\bar{a}}+\frac{N(N+2)}{4}\left(\bar{u}_{\bar{\varepsilon}}(\bar{R}, \bar{a}, x)\right)^{\frac{4}{N-2}} U_{\bar{a}}=0
$$

when $\operatorname{dist}\left(x, x_{i}\right) \leq \rho_{i}$. Hence in this ball, (61) vanishes identically. It also vanishes when $\operatorname{dist}\left(x, x_{i}\right) \geq 2 \rho_{i}$, because $\bar{u}_{\bar{\varepsilon}}(\bar{R}, \bar{a}, x)$ does not depend on $\bar{a}$ and so the difference cancels.

We come at last to the transition annulus, where $\operatorname{dist}\left(x, x_{i}\right) \in\left[\rho_{i}, 2 \rho_{i}\right]$. Recalling that $U_{\bar{a}}$ is the differential of $\bar{u}_{\bar{\varepsilon}}(\bar{R}, \bar{a}, x)$ and that $\bar{u}_{\bar{\varepsilon}}(\bar{R}, \bar{a}, x)=\chi_{i}\left(x-x_{i}\right) u_{\varepsilon_{i}}\left(R_{i}, a_{i}, x-x_{i}\right)+\left(1-\chi_{i}\right) \bar{w}_{\bar{\varepsilon}}(x)$, we write

$$
U_{\bar{a}}=\chi_{i} U_{a_{i}}^{i}+\left(1-\chi_{i}\right) W_{\bar{\varepsilon}}
$$

where $U_{a_{i}}^{i}$ and $W_{\bar{\varepsilon}}$ are the differentials of $u_{\varepsilon_{i}}$ and $\bar{w}_{\bar{\varepsilon}}$, respectively. In this annulus we can estimate

$$
\left|U_{a_{i}}^{i}\right| \leq C\left(\rho^{2-N} \varepsilon\left|S_{i}\right|+\varepsilon \rho|\alpha|\right), \quad \text { and } \quad\left|W_{\bar{\varepsilon}}\right| \leq C \varepsilon \rho^{2-N}\left|S_{i}\right| .
$$

Now write (61) as a sum of three terms, $I+I I+I I I$, where

$$
\begin{gathered}
I=\left(\Delta \chi_{i}\right)\left(U_{a_{i}}^{i}-U_{0}^{i}\right)+2 \nabla \chi_{i} \cdot \nabla\left(U_{a_{i}}^{i}-U_{0}^{i}\right), \\
I I=\chi_{i} \Delta\left(U_{a_{i}}^{i}-U_{0}^{i}\right)+\chi_{i} \frac{N(N+2)}{4}\left(\bar{u}_{\bar{\varepsilon}}(\bar{R}, \bar{a}, x)^{\frac{4}{N-2}} U_{a_{i}}^{i}-\bar{u}_{\bar{\varepsilon}}(\bar{R}, 0, x)^{\frac{4}{N-2}} U_{0}^{i}\right),
\end{gathered}
$$

and

$$
I I I=\left(1-\chi_{i}\right) \frac{N(N+2)}{4}\left(\bar{u}_{\bar{\varepsilon}}(\bar{R}, \bar{a}, x)^{\frac{4}{N-2}}-\bar{u}_{\bar{\varepsilon}}(\bar{R}, 0, x)^{\frac{4}{N-2}}\right) W_{\bar{\varepsilon}} .
$$

In the last of these, we have used that $W_{\bar{\varepsilon}}$ does not depend on $\bar{a}$.

This last term is the simplest to estimate. In fact, by Corollary 2,

$$
\left|\bar{u}_{\bar{\varepsilon}}(\bar{R}, \bar{a}, x)^{\frac{4}{N-2}}-\bar{u}_{\bar{\varepsilon}}(R, 0, x)^{\frac{4}{N-2}}\right| \leq \varepsilon^{\frac{4}{N-2}} \rho^{N-5} .
$$

Hence

$$
\|I I I\|_{0, \alpha, \nu-2,-2} \leq C \rho^{2-\nu} \varepsilon \rho^{2-N} \varepsilon^{\frac{4}{N-2}} \rho^{N-5}|\bar{S}| \leq C\left(\rho^{N+1-\nu}\right) \varepsilon|\bar{S}|,
$$

using (10).

To handle the middle term, recall that

$$
\Delta U_{a_{i}}^{i}+\frac{N(N+2)}{4} u_{\varepsilon_{i}}\left(R_{i}, a_{i}, x-x_{i}\right)^{\frac{4}{N-2}} U_{a_{i}}^{i}=0 .
$$

Using this, we rewrite $I I$ as

$\chi \frac{N(N+2)}{4}\left(\left(\bar{u}_{\bar{\varepsilon}}(\bar{R}, \bar{a}, x)^{\frac{4}{N-2}}-\bar{u}_{\varepsilon_{i}}\left(R_{i}, a_{i}, x\right)^{\frac{4}{N-2}}\right) U_{a_{i}}^{i}-\left(\bar{u}_{\bar{\varepsilon}}(\bar{R}, 0, x)^{\frac{4}{N-2}}-u_{\varepsilon_{i}}\left(R_{i}, 0, x-x_{i}\right)^{\frac{4}{N-2}}\right) U_{0}^{i}\right)$.

Since

$$
\left|\bar{u}_{\bar{\varepsilon}}(\bar{R}, \bar{a}, x)^{\frac{4}{N-2}}-u_{\varepsilon_{i}}\left(R_{i}, a_{i}, x-x_{i}\right)^{\frac{4}{N-2}}\right| \leq C \varepsilon^{\frac{4}{N-2}} \rho^{N-5}
$$

we get

$$
\|I I\|_{0, \alpha, \nu-2,-2} \leq C \rho^{2-\nu}\left(\rho^{2-N} \varepsilon|\bar{S}|+\varepsilon \rho|\bar{\alpha}|\right) \varepsilon^{\frac{4}{N-2}} \rho^{N-5} \leq \rho^{N+1-\nu}\left(\varepsilon|\bar{S}|+\varepsilon \rho^{N-1}|\bar{\alpha}|\right) .
$$

The first term, $I$, is the most difficult to estimate suitably. First observe that

$$
\begin{aligned}
& U_{a_{i}}^{i}-U_{0}^{i}=S_{i} \partial_{R_{i}}\left(u_{\varepsilon_{i}}\left(R_{i}, a_{i}, x-x_{i}\right)-u_{\varepsilon_{i}}\left(R_{i}, 0, x-x_{i}\right)\right) \\
& +\sum_{j} \alpha_{i}^{j} \partial_{a_{i}^{j}}\left(u_{\varepsilon_{i}}\left(R_{i}, a_{i}, x-x_{i}\right)-u_{\varepsilon_{i}}\left(R_{i}, 0, x-x_{i}\right)\right) .
\end{aligned}
$$


From the proof of Proposition 4 we see that

$$
u_{\varepsilon_{i}}\left(R_{i}, a_{i}, x-x_{i}\right)-u_{\varepsilon_{i}}\left(R_{i}, 0, x-x_{i}\right)=\varepsilon_{i} \frac{N-2}{2} R_{i}^{\frac{2-N}{2}} a_{i} \cdot\left(x-x_{i}\right)+O\left(\varepsilon\left|x-x_{i}\right|^{2}\right) .
$$

Noting that the derivative with respect to $a$ of the leading term of this expression is independent of $a$, hence cancels in the difference, we get

$$
\left|U_{a_{i}}^{i}-U_{0}^{i}\right| \leq C \rho(\varepsilon|\bar{S}|+\varepsilon \rho|\bar{\alpha}|), \quad \text { and } \quad\left|\nabla\left(U_{a_{i}}^{i}-U_{0}^{i}\right)\right| \leq C(\varepsilon|\bar{S}|+\varepsilon \rho|\bar{\alpha}|) .
$$

Thus we see that both terms in $I$ are of size $\rho^{-1}(\varepsilon|\bar{S}|+\varepsilon \rho|\bar{\alpha}|)$, and in particular, they are not small relative to the norm of $\tilde{\mathcal{M}}$. Fortunately, the term we actually want to estimate, $\Lambda_{\bar{\varepsilon}, \bar{R}, 0}^{-1}(I)$, is still small because $I$ has support in the union of annuli $B\left(x_{i}, 2 \rho_{i}\right) \backslash B\left(x_{i}, \rho_{i}\right)$, which has small volume. The following result will be proved in the next section.

Lemma 9 Suppose $\nu \in(1,2)$. Let $h$ be the term I. Expand it in terms of the eigenfunctions on $S^{N-1}$ :

$$
h\left(x_{i}+r \theta\right)=\sum_{j=0}^{\infty} h_{j}(r) \phi_{j}(\theta)
$$

and let

$$
h^{\prime}=\sum_{j=1}^{N} h_{j}(r) \phi_{j}(\theta) \quad \text { and } \quad h^{\prime \prime}=\sum_{j=N+1}^{\infty} h_{j}(r) \phi_{j}(\theta) .
$$

Then the solution $\hat{w}=\mathcal{L}_{\bar{\varepsilon}, \bar{R}}^{-1} h=(\hat{v}, \hat{S}, \hat{\alpha})$ satisfies

$$
\|\hat{v}\|_{2, \alpha, \nu, 2} \leq C\left(\rho^{2-\nu} \sup \left|h_{0}\right|+\rho^{2-\nu} \sup \left|h^{\prime}\right|+\left\|h^{\prime \prime}\right\|_{0, \alpha, \nu-2,-2}\right) .
$$

In addition, for any $\mu \in(-N, 2)$, we have

$$
\varepsilon\left|\hat{S}_{i}\right| \equiv\left|\mathcal{K}_{\bar{\varepsilon}, \bar{R}, i}^{0}(h)\right| \leq C\left(\rho^{2} \sup \left|h_{0}(r)\right|+\rho^{N+1} \sup \left|h^{\prime}(r)\right|+|| h^{\prime \prime}||_{0, \alpha, \mu-2,-2}\right),
$$

and, for $j=1, \ldots, N$,

$$
\varepsilon\left|\hat{\alpha}_{i}^{j}\right| \equiv\left|\mathcal{K}_{\bar{\varepsilon}, \bar{R}, i}^{j}(h)\right| \leq C\left(\rho^{2} \sup \left|h_{0}(r)\right|+\rho \sup \left|h^{\prime}(r)\right|+\left.|| h^{\prime \prime}\right|_{0, \alpha, \mu-2,-2}\right) .
$$

If $i^{\prime} \neq i$, then for $j=1, \ldots, N$,

$$
\left|\hat{\alpha}_{i^{\prime}}^{j}\right| \equiv\left|\mathcal{K}_{\bar{\varepsilon}, \bar{R}, i^{\prime}}^{j}(h)\right| \leq C\left(\rho^{2} \sup \left|h_{0}(r)\right|+\rho^{N+1} \sup \left|h^{\prime}(r)\right|+\left.|| h^{\prime \prime}\right|_{0, \alpha, \mu-2,-2}\right) .
$$

The point of this result, of course, is that because $h$ is supported in a region with small volume, one can obtain better estimates for $\mathcal{L}_{\bar{\varepsilon}, \bar{R}}^{-1} h$ (and then also for $\Lambda_{\bar{\varepsilon}, \bar{R}, 0}^{-1} h$ ) than the obvious ones. We apply this as follows. Using (65), we see that the terms in the eigenfunction expansion of $I$ satisfy

$$
\left|h_{0}\right| \leq \varepsilon(|\bar{S}|+|\bar{\alpha}|), \quad\left|h^{\prime}\right| \leq C \rho^{-1}(\varepsilon|\bar{S}|+\varepsilon \rho|\bar{\alpha}|),
$$

and

$$
\|\left. h^{\prime \prime}\right|_{0, \alpha, \mu-2,-2} \leq C \varepsilon \rho^{2-\mu}(|\bar{S}|+|\bar{\alpha}|),
$$

for any $\mu \in(-N, 2)$. We also see that

$$
\left.\rho^{\nu}|| h\right|_{0, \alpha, \nu-2,-2} \leq C\left(\rho^{2} \sup \left|h_{0}\right|+\rho^{2} \sup \left|h^{\prime}\right|+\rho^{\nu}|| h^{\prime \prime} \|_{0, \alpha, \nu-2,-2}\right) \leq C \rho(\varepsilon|\bar{S}|+\varepsilon \rho|\bar{\alpha}|) .
$$


Now apply the Lemma to find a solution $\mathcal{L}_{\bar{\varepsilon}, \bar{R}}^{-1} h=\hat{w}=(\hat{v}, \hat{S}, \hat{\alpha})$. It satisfies

and

$$
\begin{aligned}
\rho^{\nu}|| \hat{v} \|_{2, \alpha, \nu, 2} & \leq C\left(\rho ^ { 2 } \left(\varepsilon(|\bar{S}|+|\bar{\alpha}|)+\rho^{2}\left(\rho^{-1}(\varepsilon|\bar{S}|+\varepsilon \rho|\bar{\alpha}|)+\rho^{\nu}\left(\varepsilon \rho^{2-\nu}(|\bar{S}|+|\bar{\alpha}|)\right),\right.\right.\right. \\
\varepsilon|\hat{S}| & \leq C\left(\rho^{2} \varepsilon(|\bar{S}|+|\bar{\alpha}|)+\rho^{N}(\varepsilon|\bar{S}|+\varepsilon \rho|\bar{\alpha}|)+\rho^{2-\mu} \varepsilon(|\bar{S}|+|\bar{\alpha}|)\right)
\end{aligned}
$$

$$
\varepsilon \rho|\hat{\alpha}| \leq C\left(\rho^{3} \varepsilon(|\bar{S}|+|\bar{\alpha}|)+\rho^{2}\left(\rho^{-1}(\varepsilon|\bar{S}|+\varepsilon \rho|\bar{\alpha}|)\right)+\varepsilon \rho^{3-\mu}(|\bar{S}|+|\bar{\alpha}|)\right) .
$$

In the second and third expressions, we have estimated $h^{\prime \prime}$ in the $\mathcal{C}_{\mu-2,-2}^{0, \alpha}$ norm, for any $\mu \in$ $(-N, 2)$. By taking $\mu=0$, for example, we see that we can bound all three of these expressions by

$$
C \rho(\varepsilon|\bar{S}|+\varepsilon \rho|\bar{\alpha}|) .
$$

The solution $\tilde{w}=\Lambda_{\bar{\varepsilon}, \bar{R}, 0}^{-1} h$ that we are actually interested in can be estimated in terms of these quantities, using the estimates stated at the end of Step 2. Combined with the preceding, we see that

$$
\|\left.\tilde{w}\right|_{\tilde{\mathcal{M}}} \leq \rho(\varepsilon|\bar{S}|+\varepsilon \rho|\bar{\alpha}|) .
$$

We combine this estimate with the estimates $(60),(63)$ and (64), and use the uniform boundedness of $\Lambda_{\bar{\varepsilon}, \bar{R}, 0}^{-1}$ for these terms to finally conclude that the operator norm of $\Lambda_{\bar{\varepsilon}, \bar{R}, 0}^{-1}\left(\tilde{\Lambda}_{\bar{\varepsilon}, \bar{R}, \bar{a}}-\tilde{\Lambda}_{\bar{\varepsilon}, \bar{R}, 0}\right)$ on $\tilde{\mathcal{M}}$ can be made as small as desired by choosing $\varepsilon$ small. This will show that

$$
\tilde{\Lambda}_{\bar{\varepsilon}, \bar{R}, 0}^{-1} \tilde{\Lambda}_{\bar{\varepsilon}, \bar{R}, \bar{a}}=I+\tilde{\Lambda}_{\bar{\varepsilon}, \bar{R}, 0}^{-1}\left(\tilde{\Lambda}_{\bar{\varepsilon}, \bar{R}, \bar{a}}-\tilde{\Lambda}_{\bar{\varepsilon}, \bar{R}, 0}\right)
$$

is invertible, hence

$$
G=\left(I+\tilde{\Lambda}_{\bar{\varepsilon}, \bar{R}, 0}^{-1}\left(\tilde{\Lambda}_{\bar{\varepsilon}, \bar{R}, \bar{a}}-\tilde{\Lambda}_{\bar{\varepsilon}, \bar{R}, 0}\right)\right)^{-1} \tilde{\Lambda}_{\bar{\varepsilon}, \bar{R}, 0}^{-1}
$$

is a the inverse for $\tilde{\Lambda}_{\bar{\varepsilon}, \bar{R}, \bar{a}}$. Clearly it is uniformly bounded in $\varepsilon$.

Step 4. We conclude the proof of the theorem by showing that the sharper estimates for the solution of $\Lambda_{\bar{\varepsilon}, \bar{R}, \bar{a}} w=f$ are valid. We proceed as in Lemma 8. Thus, let $w_{0}=\Lambda_{\bar{\varepsilon}, \bar{R}, 0}^{-1}(f)$ and $w=(v, \bar{S}, \bar{\alpha})=\Lambda_{\bar{\varepsilon}, \bar{R}, \bar{a}}^{-1} f$. Then writing $B=\Lambda_{\bar{\varepsilon}, \bar{R}, \bar{a}}-\Lambda_{\bar{\varepsilon}, \bar{R}, 0}$, we derive

$$
\begin{gathered}
\Lambda_{\bar{\varepsilon}, \bar{R}, \bar{a}}^{-1}=\left(I+\Lambda_{\bar{\varepsilon}, \bar{R}, 0}^{-1} B\right)^{-1} \Lambda_{\bar{\varepsilon}, \bar{R}, 0}^{-1}=\left(I+\Lambda_{\bar{\varepsilon}, \bar{R}, 0}^{-1} B-\Lambda_{\bar{\varepsilon}, \bar{R}, 0}^{-1} B\right)\left(I+\Lambda_{\bar{\varepsilon}, \bar{R}, 0}^{-1} B\right)^{-1} \Lambda_{\bar{\varepsilon}, \bar{R}, 0}^{-1}= \\
\Lambda_{\bar{\varepsilon}, \bar{R}, 0}^{-1}-\Lambda_{\bar{\varepsilon}, \bar{R}, 0}^{-1} B\left(I+\Lambda_{\bar{\varepsilon}, \bar{R}, 0}^{-1} B\right)^{-1} \Lambda_{\bar{\varepsilon}, \bar{R}, 0}^{-1} .
\end{gathered}
$$

Apply this to $f$ to get

$$
w=w_{0}-\Lambda_{\bar{\varepsilon}, \bar{R}, 0}^{-1} B w_{1}
$$

where we have set $w_{1}=\left(I+\Lambda_{\bar{\varepsilon}, \bar{R}, 0}^{-1} B\right)^{-1} w_{0}$. Clearly we have

$$
\left\|w_{1}\right\|_{\tilde{\mathcal{M}}} \leq C|| w_{0} \|_{\tilde{\mathcal{M}}}
$$

We divide $w_{1}$ into a sum $v_{1}+\tilde{w}_{1}$, where we have just lumped the second and third terms in the usual decomposition into $\tilde{w}_{1}$. Thus if we let

$$
-\Lambda_{\bar{\varepsilon}, \bar{R}, 0}^{-1} B v_{1}=(\hat{v}, \hat{S}, \hat{\alpha}), \quad \text { and } \quad-\Lambda_{\bar{\varepsilon}, \bar{R}, 0}^{-1} B \tilde{w}_{1}=(\tilde{v}, \tilde{S}, \tilde{\alpha})
$$

then we have

$$
(v, \bar{S}, \bar{\alpha})=\left(v_{0}, \bar{S}_{0}, \bar{\alpha}_{0}\right)+(\hat{v}, \hat{S}, \hat{\alpha})+(\tilde{v}, \tilde{S}, \tilde{\alpha}) .
$$

We estimate these new terms in turn. First, from (60),

$$
\left\|\Lambda_{\bar{\varepsilon}, \bar{R}, 0}^{-1} B v_{1}\right\|_{\tilde{\mathcal{M}}}=\rho^{\nu}|| \hat{v}\left\|_{2, \alpha, \nu, 2}+\varepsilon|\hat{S}|+\varepsilon \rho|\hat{\alpha}| \leq C \rho^{\frac{N+2}{2}}\right\| v_{1}\left\|_{2, \alpha, \nu, 2} \leq C \rho^{\frac{N+2}{2}-\nu}\right\| w_{1} \|_{\tilde{\mathcal{M}}}
$$

We now use (67) to conclude that this term is bounded by $\rho^{\frac{N+2}{2}-\nu}\left\|w_{0}\right\|_{\tilde{\mathcal{M}}}$.

For the other term, use Lemma 9 to estimate $\left\|\Lambda_{\bar{\varepsilon}, \bar{R}, 0}^{-1} B \tilde{w}_{1}\right\|_{\mathcal{M}_{\mathcal{M}}}$ first in terms of $w_{1}$ and then $w_{0}$. Finally, substitute the sharp estimates from the end of Step 2 for $w_{0}$ in terms of $\left(v_{0}, \bar{S}_{0}, \bar{\alpha}_{0}\right)=$ $\mathcal{L}_{\bar{\varepsilon}, \bar{R}}^{-1}(f)$. We omit the details, which are straightforward and which lead to the estimates stated in the Proposition. The proof is complete. 


\section{Estimates on the error term}

In this section we shall derive estimates on the size of the error term in the approximate solution.

Lemma 10 Suppose that the parameters $R_{i}>1$ and $a_{i} \in \mathbb{R}^{N}$ satisfy the relations (12) and (13) of Proposition 4. Let $\bar{u}_{\bar{\varepsilon}}=\bar{u}_{\bar{\varepsilon}}(\bar{R}, \bar{a}, \cdot)$, and define $\zeta \equiv \Delta \bar{u}_{\bar{\varepsilon}}+\frac{N(N-2)}{4} \bar{u}_{\bar{\varepsilon}}^{\frac{N+2}{N-2}}$; then set $w=\tilde{\Lambda}_{\bar{\varepsilon}, \bar{R}, \bar{a}}^{-1} \zeta$. Then, for $\nu \in(1,2)$, and for some constant $C$ independent of $\varepsilon$,

$$
\|w\|_{\tilde{\mathcal{M}}} \leq C \varepsilon \rho^{2} .
$$

Proof : Write $\mathcal{L}_{\bar{\varepsilon}, \bar{R}}^{-1} \zeta=(v, \bar{S}, \bar{\alpha})$ as usual. Clearly, it is sufficient to prove that $\|(v, \bar{S}, \bar{\alpha})\|_{\tilde{\mathcal{M}}} \leq$ $C \varepsilon \rho^{2}$. The estimate for $v$ is rather straightforward; however, the estimates for $\bar{S}$ and $\bar{\alpha}$ are more delicate, since they rely on the particular structure of $\zeta$, and so we need to follow the construction of $\mathcal{L}_{\bar{\varepsilon}, \bar{R}}^{-1}$ in Proposition 6 step by step.

Step 1. From the definition of $\bar{u}_{\bar{\varepsilon}}$, we see that in $\mathbb{R}^{N} \backslash \cup_{i=1}^{n} B\left(x_{i}, 2 \rho_{i}\right)$

$$
\zeta(x)=\frac{N(N-2)}{4} \bar{u}_{\bar{\varepsilon}}^{\frac{N+2}{N-2}}(x),
$$

and so

$$
|\zeta(x)| \leq C \varepsilon^{\frac{N+2}{N-2}} \operatorname{dist}(x, \Sigma)^{-N-2} .
$$

In particular, in $\Omega=\mathbb{R}^{N} \backslash \cup_{i=1}^{n} \overline{B\left(x_{i}, 1\right)},\|\zeta\|_{0, \alpha, \nu-2,-2} \leq C \varepsilon^{\frac{N+2}{N-2}}$. From Proposition 7, there exists a solution of

$$
\left\{\begin{array}{rlll}
\mathcal{L}_{\bar{\varepsilon}, \bar{R}} w_{\text {ext }}=\zeta & \text { in } & \Omega \\
w_{\text {ext }} & =0 & \text { on } & \partial \Omega
\end{array}\right.
$$

which satisfies

$$
\left\|w_{\text {ext }}\right\|_{2, \alpha, \nu, 2} \leq C \varepsilon^{\frac{N+2}{N-2}} .
$$

Step 2. Our task now is to estimate $\zeta$ in each $B\left(x_{i}, 1\right)$. In this step, we consider its eigenfunction expansion,

$$
\zeta(x)=\sum_{j=0}^{+\infty} \zeta_{j}^{i}(r) \phi_{j}(\theta),
$$

where $r=\left|x-x_{i}\right|$, and estimate the terms in three separate regions.

From the definition of $\bar{u}_{\bar{\varepsilon}}$, we see that inside each $B\left(x_{i}, \rho_{i}\right), \zeta=\Delta \bar{u}_{\bar{\varepsilon}}+\frac{N(N-2)}{4} \bar{u}_{\bar{\varepsilon}}^{\frac{N+2}{N-2}}=0$, and so all $\zeta_{j}^{i}=0$.

Next, $\zeta=\frac{N(N-2)}{4} \bar{u}_{\overline{\bar{\varepsilon}}}^{\frac{N+2}{N-2}}$ in $B\left(x_{i}, 1\right) \backslash B\left(x_{i}, 2 \rho_{i}\right)$. Thus

$$
\left|\zeta_{0}^{i}(r)\right| \leq c \varepsilon^{\frac{N+2}{N-2}} r^{-(N+2)}, \quad \sum_{j=1}^{N}\left|\zeta_{j}^{i}(r)\right| \leq c \varepsilon^{\frac{N+2}{N-2}} r^{-3},
$$

and

$$
\left|\sum_{j=N+1}^{+\infty} \zeta_{j}^{i}(r) \phi_{j}(\theta)\right| \leq C \varepsilon^{\frac{N+2}{N-2}} r^{-2} .
$$

Finally, in $B\left(x_{i}, 2 \rho_{i}\right) \backslash B\left(x_{i}, \rho_{i}\right)$, we have

$$
\begin{aligned}
& \zeta(x)=2\left(\partial_{r_{i}} \chi_{i}\right)\left(\partial_{r_{i}} u_{\varepsilon_{i}}\left(R_{i}, a_{i}, x-x_{i}\right)-\partial_{r_{i}} \bar{w}_{\bar{\varepsilon}}(\bar{R}, x)\right)+\left(\Delta \chi_{i}\right)\left(u_{\varepsilon_{i}}\left(R_{i}, a_{i}, x-x_{i}\right)-\bar{w}_{\bar{\varepsilon}}(\bar{R}, x)\right) \\
& -\frac{N(N-2)}{4}\left(\chi_{i}\left(u_{\varepsilon_{i}}\left(R_{i}, a_{i}, x-x_{i}\right)\right)^{\frac{N+2}{N-2}}-\left(\chi_{i} u_{\varepsilon_{i}}\left(R_{i}, a_{i}, x-x_{i}\right)+\left(1-\chi_{i}\right) \bar{w}_{\bar{\varepsilon}}(\bar{R}, x)\right)^{\frac{N+2}{N-2}}\right) .
\end{aligned}
$$


From this we get the estimates

$$
\sum_{j=0}^{N}\left|\zeta_{j}^{i}(r)\right|+\left|\sum_{j \geq N+1} \zeta_{j}^{i}(r) \phi_{j}(\theta)\right| \leq C \varepsilon
$$

Step 3. By Proposition 5, we get, for $i=1, \ldots, n$, solutions of the problem

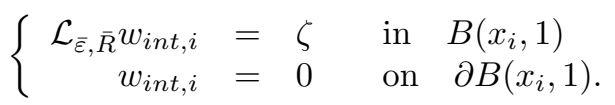

These can be expanded as

$$
w_{i n t, i}=G_{\varepsilon_{i}, R_{i}}\left(\zeta_{\mid B\left(x_{i}, 1\right)}\right)+\sum_{j=0}^{N} K_{\varepsilon_{i}, R_{i}}^{j}\left(\zeta_{\mid B\left(x_{i}, 1\right)}\right) \frac{1}{\varepsilon_{i}} \Psi_{\varepsilon_{i}}^{j,+}\left(\left(x-x_{i}\right) / R_{i}\right) .
$$

We can also consider the eigenfunction decomposition of $w_{i n t, i}$

$$
w_{\text {int }_{i}}=\sum_{j=0}^{\infty} w_{j}(r) \phi_{j}(\theta)
$$

where $r=\left|x-x_{i}\right|$. We now estimate the different terms in this formula.

First consider the solution $w_{0}^{i}$ in the $j=0$ eigencomponent. We can represent $w_{0}^{i}$ explicitly in terms of $\zeta_{0}^{i}$ using the variation of constants formula. Write

$$
\psi_{\varepsilon_{i}, R_{i}}^{0,+}(r)=r^{\frac{2-N}{2}} \frac{\partial v_{\varepsilon_{i}}}{\partial t}\left(-\log \left(r / R_{i}\right)\right)=r^{\frac{2-N}{2}} \Phi_{\varepsilon_{i}}^{0,+}\left(-\log \left(r / R_{i}\right)\right)
$$

Using Proposition 3 , and the fact that $R_{i} \geq 1+c_{0}$, we see that, up to a constant, $\psi_{\varepsilon_{i}, R_{i}}^{0,+} \sim \varepsilon r^{2-N}$ for $\rho_{i} \leq r \leq 1$. In particular, this function never vanishes. This means that we can write

$$
w_{0}^{i}(r)=K_{\varepsilon_{i}, R_{i}}^{0}\left(\zeta_{\mid B\left(x_{i}, 1\right)}\right) \frac{1}{\varepsilon_{i}} \Psi_{\varepsilon_{i}}^{0,+}\left(\left(x-x_{i}\right) / R_{i}\right)+\tilde{w}_{0}^{i}(r) .
$$

where

$$
\tilde{w}_{0}^{i}(r)=\psi_{\varepsilon_{i}, R_{i}}^{0,+}(r) \int_{\rho_{i}}^{r}\left(\psi_{\varepsilon_{i}, R_{i}}^{0,+}(s)\right)^{-2} s^{1-N} \int_{\rho_{i}}^{s} \psi_{\varepsilon_{i}, R_{i}}^{0,+}(t) t^{N-1} \zeta_{0}^{i}(t) d t d s
$$

and the first term in the decomposition of $w_{0}^{i}$ is intended to correct the boundary value. From the bounds above on $\zeta_{0}^{i}$, and using the approximation above for $\psi_{\varepsilon_{i}, R_{i}}^{0,+}$, we find that

$$
\left|\tilde{w}_{0}^{i}\right| \leq C \varepsilon \rho^{2} \quad \text { for all } \quad \rho_{i} \leq\left|x-x_{i}\right| \leq 1
$$

Therefore, since $\varepsilon_{i}^{-1} \psi_{\varepsilon_{i}, R_{i}}^{0,+}$ is bounded above and below for $r=1$, we also get

$$
\left|K_{\varepsilon_{i}, R_{i}}^{0}\left(\zeta_{\mid B\left(x_{i}, 1\right)}\right)\right| \leq c \varepsilon \rho^{2} .
$$

We also require an estimate for the normal derivative of $w_{0}^{i}$ on $\partial B\left(x_{i}, 1\right)$. To get this, we use the slightly different representation

$$
w_{0}^{i}(r)=\psi_{\varepsilon_{i}, R_{i}}^{0,+}(r) \int_{r}^{1}\left(\psi_{\varepsilon_{i}, R_{i}}^{0,+}(s)\right)^{-2} s^{1-N} \int_{s}^{\rho_{i}} \psi_{\varepsilon_{i}, R_{i}}^{0,+}(t) t^{N-1} \zeta_{0}^{i}(t) d t d s .
$$

Differentiating this gives

$$
\left|\partial_{r} w_{0}^{i}(1)\right| \leq\left(\psi_{\varepsilon_{i}, R_{i}}^{0,+}(1)\right)^{-1} \int_{\rho_{i}}^{1} \psi_{\varepsilon_{i}, R_{i}}^{0,+}(t) t^{N-1}\left|\zeta_{0}^{i}(t)\right| d t
$$


and so

$$
\left|\partial_{r_{i}} w_{0}^{i}(1)\right| \leq c \varepsilon \rho^{2} .
$$

For $j=1, \ldots, N$, we may perform a similar analysis. As before, $w_{j}^{i}$ denotes the component of the solution in the $j^{\text {th }}$ eigencomponent, as

$$
w_{j}^{i}(r)=K_{\varepsilon_{i}, R_{i}}^{j}\left(\zeta_{\mid B\left(x_{i}, 1\right)}\right) \frac{1}{\varepsilon_{i}} \Psi_{\varepsilon_{i}}^{j,+}\left(\left(x-x_{i}\right) / R_{i}\right)+\tilde{w}_{j}^{i}(r)
$$

where, denoting by

$$
\psi_{\varepsilon_{i}, R_{i}}^{1,+}(r)=r^{\frac{4-N}{2}}\left(\frac{N-2}{2} v_{\varepsilon_{i}}\left(-\log \left(r / R_{i}\right)\right)-\frac{\partial v_{\varepsilon_{i}}}{\partial t}\left(-\log \left(r / R_{i}\right)\right)\right)=r^{\frac{2-N}{2}} \Phi_{\varepsilon_{i}}^{1,+}\left(-\log \left(r / R_{i}\right)\right),
$$

we represent

$$
\tilde{w}_{j}^{i}(r)=\psi_{\varepsilon_{i}, R_{i}}^{1,+}(r) \int_{\rho_{i}}^{r}\left(\psi_{\varepsilon_{i}, R_{i}}^{1,+}(s)\right)^{-2} s^{1-N} \int_{\rho_{i}}^{s} \psi_{\varepsilon_{i}, R_{i}}^{1,+}(t) \zeta_{j}^{i}(t) t^{N-1} d t d s .
$$

The estimates

$$
\left|\tilde{w}_{j}^{i}\right| \leq C \varepsilon \rho r \quad \text { and } \quad\left|K_{\varepsilon_{i}, R_{i}}^{j}\left(\zeta_{\mid B\left(x_{i}, 1\right)}\right)\right| \leq C \varepsilon \rho
$$

follow as above, using the fact that $\psi_{\varepsilon_{i}, R_{i}}^{1,+} \sim \varepsilon r$ in $\left[\rho_{i}, 1\right]$.

The estimate for the normal derivative follows from the alternate representation

$$
w_{j}^{i}(r)=\psi_{\varepsilon_{i}, R_{i}}^{1,+}(r) \int_{r}^{1}\left(\psi_{\varepsilon_{i}, R_{i}}^{1,+}(s)\right)^{-2} s^{1-N} \int_{s}^{\rho_{i}} \psi_{\varepsilon_{i}, R_{i}}^{1,+}(t) t^{N-1} \zeta_{j}^{i}(t) d t d s
$$

which yields

$$
\left|\partial_{r_{i}} w_{j}^{i}(r)\right| \leq\left(\psi_{\varepsilon_{i}, R_{i}}^{1,+}(1)\right)^{-1} \int_{\rho_{i}}^{1} \psi_{\varepsilon_{i}, R_{i}}^{1,+}(t) t^{N-1}\left|\zeta_{j}^{i}(t)\right| d t .
$$

The estimate

$$
\left|\partial_{r_{i}} w_{j}^{i}(1)\right| \leq c \varepsilon \rho^{N+1},
$$

follows immediately.

Let $\tilde{\zeta}^{i}$ denote the sum over all higher eigencomponents of $\zeta$. From (69) and (70), and from Proposition 5, for each $\mu \in(-N, 2)$,

$$
\left\|G_{\varepsilon_{i}, R_{i}}\left(\tilde{\zeta}^{i}\right)\right\|_{2, \alpha, \mu} \leq C \varepsilon \rho^{2-\mu},
$$

where $C$ is independent of $\varepsilon$. In particular, using this with $\mu=\nu$, and also using (71) and (73), we get

$$
\left\|G_{\varepsilon_{i}, R_{i}}\left(\zeta_{\mid B\left(x_{i}, 1\right)}\right)\right\|_{2, \alpha, \nu} \leq C \varepsilon \rho^{2-\nu} .
$$

Finally, take $\mu=0$ in (75) to get the estimate

$$
\left\|\partial_{r_{j}} G_{\varepsilon_{i}, R_{i}}\left(\tilde{\zeta}^{i}\right)\right\|_{\mathcal{C}^{1, \alpha}\left(\partial B\left(x_{i}, 1\right)\right)} \leq c \varepsilon \rho^{2}
$$

on the boundary of $B\left(x_{i}, 1\right)$.

Step 4. The final step is to consider the size of the correction term $w_{k e r}$, which is chosen to make the Dirichlet and Neumann data match. Its size is regulated by the size of the jump of the Neumann data of the interior and exterior solutions. Specifically, its Dirichlet data $\Psi=$ $\left(\psi_{1}, \ldots, \psi_{n}\right) \in \oplus_{i=1}^{n} \mathcal{C}^{1, \alpha}\left(\partial B\left(x_{i}, 1\right)\right)$ is chosen to satisfy

$$
\left(\mathcal{S}_{\bar{\varepsilon}, \bar{R}}-\mathcal{T}_{\bar{\varepsilon}, \bar{R}}\right)(\Psi)=\left\{\left.\left(\partial_{r_{1}} w_{i n t, 1}-\partial_{r_{1}} w_{e x t}\right)\right|_{r_{1}=1}, \ldots,\left.\left(\partial_{r_{n}} w_{i n t, n}-\partial_{r_{n}} w_{e x t}\right)\right|_{r_{n}=1}\right\} .
$$


Using (72), (74) and (76), we can estimate that the norm of the right side is bounded by $C \varepsilon \rho^{2}$. Hence $\|\Psi\|_{\mathcal{C}^{2, \alpha}} \leq C \varepsilon \rho^{2}$ as well. The estimate $\|(v, \bar{S}, \bar{\alpha})\|_{\tilde{\mathcal{M}}} \leq C \varepsilon \rho^{2}$ then follows easily.

Proof of Lemma 9: The details of this proof are very similar to those of the proof above. In fact, proceeding through the steps of the previous proof, we find first that $w_{\text {ext }}=0$. The solutions $w_{\text {int }, i}$ may be found by the same procedure. The integral representations of $w_{j}^{i}$ show that for $j=0$ and $j=1, \ldots, N$, the $\mathcal{C}_{\nu, 2}^{2, \alpha}$ norms of these terms may be bounded by $\rho^{2-\nu} \sup \left|h_{0}\right|$ and $\rho^{2-\nu}\left|\sup h^{\prime}\right|$, respectively. The remaining details are left to the reader.

\section{The nonlinear fixed point argument}

We are now in a position to find a solution of the nonlinear problem. As usual, we fix the singular set $\Sigma=\left\{x_{1}, \ldots, x_{n}\right\}$ and positive parameters $q_{1}, \ldots, q_{n}>0$ associated to a set of admissible Delaunay parameters $\bar{\varepsilon}$. We also fix an approximate solution $\bar{u}_{\bar{\varepsilon}}(\bar{R}, \bar{a}, x)$ associated to the data $\bar{R}$ and $\bar{a}$ satisfying (12) and (13). The perturbation of $\bar{u}_{\bar{\varepsilon}}(\bar{R}, \bar{a}, x)$ to an exact solution will involve not only a decaying term, but also a slight adjustment of the parameters $\bar{R}$ and $\bar{a}$. Thus, recalling that an element $w$ in $\tilde{\mathcal{M}}$ has components $(v, \bar{S}, \bar{\alpha})$, and changing our previous notation slightly, we wish to solve the equation $\mathcal{N}(w)=0$ where by definition

$$
\mathcal{N}(w) \equiv \Delta\left(\bar{u}_{\bar{\varepsilon}}(\bar{R}+\bar{S}, \bar{a}+\bar{\alpha}, \cdot)+v\right)+\frac{N(N-2)}{4}\left(\bar{u}_{\bar{\varepsilon}}(\bar{R}+\bar{S}, \bar{a}+\bar{\alpha}, \cdot)+v\right)^{\frac{N+2}{N-2}} .
$$

Recalling the linearization $\tilde{\Lambda}_{\bar{\varepsilon}, \bar{R}, \bar{a}}$ of $\mathcal{N}$ at $w=0$, we can rewrite (77), using a Taylor expansion, as

$$
\begin{gathered}
\mathcal{N}(w)=\mathcal{N}(0)+\tilde{\Lambda}_{\bar{\varepsilon}, \bar{R}, \bar{a}} w+\int_{0}^{1}\left(D \mathcal{N}_{\mid(t v, \bar{S}, \bar{\alpha})}-D \mathcal{N}_{\mid(0, \bar{S}, \bar{\alpha})}\right)(v, 0,0) d t \\
+\left(D \mathcal{N}_{\mid(0, \bar{S}, \bar{\alpha})}-D \mathcal{N}_{\mid(0,0,0)}\right)(v, 0,0)+\int_{0}^{1}\left(D \mathcal{N}_{\mid(0, t \bar{S}, t \bar{\alpha})}-D \mathcal{N}_{\mid(0,0,0)}\right)(0, \bar{S}, \bar{\alpha}) d t
\end{gathered}
$$

We have used a somewhat more elaborate expression than usual for the remainder term; this is necessary in the estimates below. Therefore, the equation $\mathcal{N}(w)=0$ can be written as

$$
\begin{gathered}
w=-\tilde{\Lambda}_{\bar{\varepsilon}, \bar{R}, \bar{a}}^{-1} \mathcal{N}(0)-\tilde{\Lambda}_{\bar{\varepsilon}, \bar{R}, \bar{a}}^{-1}\left(\int_{0}^{1}\left(D \mathcal{N}_{\mid(t v, \bar{S}, \bar{\alpha})}-D \mathcal{N}_{\mid(0, \bar{S}, \bar{\alpha})}\right)(v, 0,0) d t\right. \\
\left.+\left(D \mathcal{N}_{\mid(0, \bar{S}, \bar{\alpha})}-D \mathcal{N}_{\mid(0,0,0)}\right)(v, 0,0)+\int_{0}^{1}\left(D \mathcal{N}_{\mid(0, t \bar{S}, t \bar{\alpha})}-D \mathcal{N}_{\mid(0,0,0)}\right)(0, \bar{S}, \bar{\alpha}) d t\right) .
\end{gathered}
$$

We define the mapping $\mathcal{P}: \tilde{\mathcal{M}} \longrightarrow \tilde{\mathcal{M}}$ by

$$
\begin{gathered}
\mathcal{P}(w)=-\tilde{\Lambda}_{\bar{\varepsilon}, \bar{R}, \bar{a}}^{-1} \mathcal{N}(0)-\tilde{\Lambda}_{\bar{\varepsilon}, \bar{R}, \bar{a}}^{-1}\left(\int_{0}^{1}\left(D \mathcal{N}_{\mid(t v, \bar{S}, \bar{\alpha})}-D \mathcal{N}_{\mid(0, \bar{S}, \bar{\alpha})}\right)(v, 0,0) d t\right. \\
\left.+\left(D \mathcal{N}_{\mid(0, \bar{S}, \bar{\alpha})}-D \mathcal{N}_{\mid(0,0,0)}\right)(v, 0,0)+\int_{0}^{1}\left(D \mathcal{N}_{\mid(0, t \bar{S}, t \bar{\alpha})}-D \mathcal{N}_{\mid(0,0,0)}\right)(0, \bar{S}, \bar{\alpha}) d t\right) .
\end{gathered}
$$

We shall prove that for $\varepsilon$ small enough, $\mathcal{P}$ is a contraction on a small ball in $\tilde{\mathcal{M}}$ of radius $C_{0} \varepsilon \rho^{2}$, where $C_{0}$ is twice the constant defined in Lemma 10. This will give the existence of a solution of $\mathcal{N}(w)=0$ in the space $\tilde{\mathcal{M}}$.

Let $w_{1}, w_{2}$ be two elements in this ball. We begin by estimating the image by $\tilde{\Lambda}_{\bar{\varepsilon}, \bar{R}, \bar{a}}^{-1}$ of

$$
I\left(w_{1}, w_{2}\right) \equiv\left(D \mathcal{N}_{\mid\left(v_{2}, \bar{S}_{2}, \bar{\alpha}_{2}\right)}-D \mathcal{N}_{\mid\left(0, \bar{S}_{2}, \bar{\alpha}_{2}\right)}\right)\left(v_{2}, 0,0\right)-\left(D \mathcal{N}_{\mid\left(v_{1}, \bar{S}_{1}, \bar{\alpha}_{1}\right)}-D \mathcal{N}_{\mid\left(0, \bar{S}_{1}, \bar{\alpha}_{1}\right)}\right)\left(v_{1}, 0,0\right)
$$


Lemma 11 There exists a constant $c>0$ (depending on $C_{0}$ ) such that

$$
\left\|\tilde{\Lambda}_{\bar{\varepsilon}, \bar{R}, \bar{a}}^{-1} I\left(w_{1}, w_{2}\right)\right\|_{\tilde{\mathcal{M}}} \leq c \rho^{2-\nu}\left\|w_{2}-w_{1}\right\|_{\tilde{\mathcal{M}}}
$$

Proof : From the structure of $\mathcal{N}(w)$ we see that

$$
\begin{gathered}
\left(D \mathcal{N}_{\mid(v, \bar{S}, \bar{\alpha})}-D \mathcal{N}_{\mid(0, \bar{S}, \bar{\alpha})}\right)(v, 0,0) \\
=\frac{N(N+2)}{4}\left(\left(\bar{u}_{\bar{\varepsilon}}(\bar{R}+\bar{S}, \bar{a}+\bar{\alpha}, \cdot)+v\right)^{\frac{4}{N-2}}-\left(\bar{u}_{\bar{\varepsilon}}(\bar{R}+\bar{S}, \bar{a}+\bar{\alpha}, \cdot)\right)^{\frac{4}{N-2}}\right) v .
\end{gathered}
$$

We write

$$
I\left(w_{1}, w_{2}\right)(x)=\frac{N(N+2)}{4}\left(I_{1}+I_{2}+I_{3}\right),
$$

where

$$
\begin{gathered}
I_{1}=\left(\left(\bar{u}_{\bar{\varepsilon}}\left(\bar{R}+\bar{S}_{2}, \bar{a}+\bar{\alpha}_{2}, x\right)+v_{2}(x)\right)^{\frac{4}{N-2}}-\left(\bar{u}_{\bar{\varepsilon}}\left(\bar{R}+\bar{S}_{2}, \bar{a}+\bar{\alpha}_{2}, x\right)\right)^{\frac{4}{N-2}}\right)\left(v_{2}-v_{1}\right)(x) \\
\quad \frac{4}{N-2} v_{2}(x)\left(v_{2}-v_{1}\right)(x) \int_{0}^{1}\left(\bar{u}_{\bar{\varepsilon}}\left(\bar{R}+\bar{S}_{2}, \bar{a}+\bar{\alpha}_{2}, x\right)+s v_{2}(x)\right)^{\frac{6-N}{N-2}} d s \\
I_{2}=\left(\left(\bar{u}_{\bar{\varepsilon}}\left(\bar{R}+\bar{S}_{2}, \bar{a}+\bar{\alpha}_{2}, x\right)+v_{2}\right)^{\frac{4}{N-2}}-\left(\bar{u}_{\bar{\varepsilon}}\left(\bar{R}+\bar{S}_{1}, \bar{a}+\bar{\alpha}_{1}, x\right)+v_{1}\right)^{\frac{4}{N-2}}\right) v_{1}(x) \\
=\frac{4}{N-2} v_{1}(x) \int_{0}^{1}\left(\left(\bar{u}_{\bar{\varepsilon}}\left(\bar{R}+s \bar{S}_{2}+(1-s) \bar{S}_{1}, \bar{a}+s \bar{\alpha}_{2}+(1-s) \bar{\alpha}_{1}, x\right)+s v_{2}(x)+(1-s) v_{1}(x)\right)^{\frac{6-N}{N-2}}\right. \\
\times\left(\sum_{i} \mu_{i}\left(\bar{R}+s \bar{S}_{2}+(1-s) \bar{S}_{1}, \bar{a}+s \bar{\alpha}_{2}+(1-s) \bar{\alpha}_{1}, x\right)\left(\bar{S}_{2, i}-\bar{S}_{1, i}\right)\right. \\
\left.+\sum_{i, j} \gamma_{i}^{j}\left(\bar{R}+s \bar{S}_{2}+(1-s) \bar{S}_{1}, \bar{a}+s \bar{\alpha}_{2}+(1-s) \bar{\alpha}_{1}, x\right)\left(\bar{\alpha}_{2, i}^{j}-\bar{\alpha}_{1, i}^{j}\right)+s\left(v_{2}(x)-v_{1}(x)\right)\right) d s
\end{gathered}
$$

and finally

$$
\begin{aligned}
& I_{3}=-\left(\left(\bar{u}_{\bar{\varepsilon}}\left(\bar{R}+\bar{S}_{2}, \bar{a}+\bar{\alpha}_{2}, x\right)\right)^{\frac{4}{N-2}}-\left(\bar{u}_{\bar{\varepsilon}}\left(\bar{R}+\bar{S}_{1}, \bar{a}+\bar{\alpha}_{1}, x\right)\right)^{\frac{4}{N-2}}\right) v_{1}(x) \\
= & -\frac{4}{N-2} v_{1}(x) \int_{0}^{1}\left(\left(\bar{u}_{\bar{\varepsilon}}\left(\bar{R}+s \bar{S}_{2}+(1-s) \bar{S}_{1}, \bar{a}+s \bar{\alpha}_{2}+(1-s) \bar{\alpha}_{1}, x\right)\right)^{\frac{6-N}{N-2}}\right. \\
& \left(\sum_{i} \mu_{i}\left(\bar{R}+s \bar{S}_{2}+(1-s) \bar{S}_{1}, \bar{a}+s \bar{\alpha}_{2}+(1-s) \bar{\alpha}_{1}, x\right)\left(\bar{S}_{2, i}-\bar{S}_{1, i}\right)\right. \\
+ & \left.\sum_{i, j} \gamma_{i}^{j}\left(\bar{R}+s \bar{S}_{2}+(1-s) \bar{S}_{1}, \bar{a}+s \bar{\alpha}_{2}+(1-s) \bar{\alpha}_{1}, x\right)\left(\bar{\alpha}_{2, i}^{j}-\bar{\alpha}_{1, i}^{j}\right)\right) d s .
\end{aligned}
$$

- First consider the region where $\operatorname{dist}(x, \Sigma) \leq \varepsilon^{\frac{2}{N-2}}$. Here we use the fact that for some constant $c>0$ depending only on $N$, we have the estimates

$\left|\mu_{i}(\bar{R}+\bar{S}, \bar{a}+\bar{\alpha})\right| \leq c \bar{u}_{\bar{\varepsilon}}(\bar{R}+\bar{S}, \bar{a}+\bar{\alpha}) \quad$ and $\quad\left|\gamma_{i}^{j}(\bar{R}+\bar{S}, \bar{a}+\bar{\alpha})\right| \leq c \operatorname{dist}(x, \Sigma) \bar{u}_{\bar{\varepsilon}}(\bar{R}+\bar{S}, \bar{a}+\bar{\alpha})$, where the constant $c>0$ depends on $C_{0}$. These yield

$$
\begin{aligned}
\left|I_{1}\right| \leq & c \varepsilon \rho^{2-\nu} \sup \left(1, \varepsilon^{\frac{6-N}{N-2}}\right)|| v_{2}-v_{1} \|_{2, \alpha, \nu, 2} \operatorname{dist}(x, \Sigma)^{\frac{N-6}{2}+2 \nu} \\
\left|I_{2}\right| \leq & c \varepsilon \rho^{2-\nu}\left(\operatorname{dist}(x, \Sigma)^{\nu-2}\left(\left|\bar{S}_{2}-\bar{S}_{1}\right|+\operatorname{dist}(x, \Sigma)\left|\bar{\alpha}_{2}-\bar{\alpha}_{1}\right|\right)\right. \\
& \left.+\sup \left(1, \varepsilon^{\frac{6-N}{N-2}}\right) \operatorname{dist}(x, \Sigma)^{\frac{N-6}{2}+2 \nu}\left\|v_{2}-v_{1}\right\|_{2, \alpha, \nu, 2}\right)
\end{aligned}
$$

and

$$
\left|I_{3}\right| \leq c \varepsilon \rho^{2-\nu}\left(\operatorname{dist}(x, \Sigma)^{\nu-2}\left(\left|\bar{S}_{2}-\bar{S}_{1}\right|+\operatorname{dist}(x, \Sigma)\left|\bar{\alpha}_{2}-\bar{\alpha}_{1}\right|\right)\right.
$$


- Next, assume that $\operatorname{dist}(x, \Sigma) \in\left[\varepsilon^{\frac{2}{N-2}}, 1\right]$. In this case we use the estimates

$$
\left|\mu_{i}(\bar{R}+\bar{S}, \bar{a}+\bar{\alpha})\right| \leq c \varepsilon \operatorname{dist}(x, \Sigma)^{2-N}
$$

and

$$
\left|\gamma_{i}^{j}(\bar{R}+\bar{S}, \bar{a}+\bar{\alpha})\right| \leq c \varepsilon \operatorname{dist}(x, \Sigma)\left(1+\varepsilon^{\frac{4}{N-2}} \operatorname{dist}(x, \Sigma)^{-N}\right),
$$

where the constant $c>0$ depends on $C_{0}$. Then as above, we derive

$$
\begin{gathered}
\left|I_{1}\right| \leq c \varepsilon^{\frac{4}{N-2}} \rho^{2-\nu}\left\|v_{2}-v_{1}\right\|_{2, \alpha, \nu, 2} \operatorname{dist}(x, \Sigma)^{N-6+2 \nu} \\
\left|I_{2}\right| \leq c \varepsilon \rho^{2-\nu}\left(\varepsilon^{\frac{4}{N-2}} \operatorname{dist}(x, \Sigma)^{\nu-4}\left(\left|\bar{S}_{2}-\bar{S}_{1}\right|+\left(\operatorname{dist}(x, \Sigma)^{N-1}+\varepsilon^{\frac{4}{N-2}} \operatorname{dist}(x, \Sigma)^{-1}\right)\left|\bar{\alpha}_{2}-\bar{\alpha}_{1}\right|\right)\right. \\
\left.+\varepsilon^{\frac{6-N}{N-2}} \operatorname{dist}(x, \Sigma)^{N-6+2 \nu}\left\|v_{2}-v_{1}\right\|_{2, \alpha, \nu, 2}\right)
\end{gathered}
$$

and

$$
\left|I_{3}\right| \leq c \varepsilon^{\frac{N+2}{N-2}} \rho^{2-\nu}\left(\operatorname{dist}(x, \Sigma)^{\nu-4}\left(\left|\bar{S}_{2}-\bar{S}_{1}\right|+\left(\operatorname{dist}(x, \Sigma)^{N-1}+\varepsilon^{\frac{4}{N-2}} \operatorname{dist}(x, \Sigma)^{-1}\right)\left|\bar{\alpha}_{2}-\bar{\alpha}_{1}\right|\right) .\right.
$$

- Finally, if $\operatorname{dist}(x, \Sigma) \geq 1$, we obtain

$$
\begin{gathered}
\left|I_{1}\right| \leq c \varepsilon^{\frac{4}{N-2}} \rho^{2-\nu}\left\|v_{2}-v_{1}\right\|_{2, \alpha, \nu, 2} \operatorname{dist}(x, \Sigma)^{-N-2} \\
\left|I_{2}\right| \leq c \varepsilon \rho^{2-\nu}\left(\varepsilon^{\frac{4}{N-2}}\left|\bar{S}_{2}-\bar{S}_{1}\right|+\varepsilon^{\frac{6-N}{N-2}}\left\|v_{2}-v_{1}\right\|_{2, \alpha, \nu, 2}\right) \operatorname{dist}(x, \Sigma)^{-N-2}
\end{gathered}
$$

and

$$
\left|I_{3}\right| \leq c \varepsilon^{\frac{N+2}{N-2}} \rho^{2-\nu}\left|\bar{S}_{2}-\bar{S}_{1}\right| \operatorname{dist}(x, \Sigma)^{-N-2} .
$$

Combining these gives

$$
\left\|I\left(w_{1}, w_{2}\right)\right\|_{0, \alpha, \nu-2,-2} \leq c \rho^{2-\nu}\left(\varepsilon^{\frac{4}{N-2}}|| v_{2}-\left.v_{1}\right|_{2, \alpha, \nu, 2}+\varepsilon\left(\left|\bar{S}_{2}-\bar{S}_{1}\right|+\varepsilon^{\frac{2}{N-2}}\left|\bar{\alpha}_{2}-\bar{\alpha}_{1}\right|\right)\right) .
$$

In particular

$$
\begin{gathered}
\|\left. I\left(w_{1}, w_{2}\right)\right|_{0, \alpha, \nu-2,-2} \leq c \rho^{2-\nu}\left(\rho^{\nu}|| v_{2}-v_{1} \|_{2, \alpha, \nu, 2}+\varepsilon\left|\bar{S}_{2}-\bar{S}_{1}\right|+\varepsilon \rho\left|\bar{\alpha}_{2}-\bar{\alpha}_{1}\right|\right) \\
=c \rho^{2-\nu}|| w_{2}-w_{1} \|_{\tilde{\mathcal{M}}}
\end{gathered}
$$

The estimate of the Lemma now follows from the boundedness of $\Lambda_{\bar{\varepsilon}, \bar{R}, \bar{a}}^{-1}$, as proved in Proposition 9 .

Next shall estimate the image by $\tilde{\Lambda}_{\bar{\varepsilon}, \bar{R}, \bar{a}}^{-1}$ of

$$
I I\left(w_{1}, w_{2}\right)=\left(D \mathcal{N}_{\mid\left(0, \bar{S}_{2}, \bar{\alpha}_{2}\right)}-D \mathcal{N}_{\mid(0,0,0)}\right)\left(v_{2}, 0,0\right)-\left(D \mathcal{N}_{\mid\left(0, \bar{S}_{1}, \bar{\alpha}_{1}\right)}-D \mathcal{N}_{\mid(0,0,0)}\right)\left(v_{1}, 0,0\right) .
$$

Lemma 12 There exists some constant $c>0$ (depending on $C_{0}$ ) such that

$$
\left\|\tilde{\Lambda}_{\bar{\varepsilon}, \bar{R}, \bar{a}}^{-1} I I\left(w_{1}, w_{2}\right)\right\|_{\tilde{\mathcal{M}}} \leq c \rho^{2-\nu}\left\|w_{2}-w_{1}\right\|_{\tilde{\mathcal{M}}}
$$

Proof : Recall that

$$
\left(D \mathcal{N}_{\mid(0, \bar{S}, \bar{\alpha})}-D \mathcal{N}_{\mid(0,0,0)}\right)(v, 0,0)=\frac{N(N+2)}{4}\left(\left(\bar{u}_{\bar{\varepsilon}}(\bar{R}+\bar{S}, \bar{a}+\bar{\alpha}, \cdot)\right)^{\frac{4}{N-2}}-\left(\bar{u}_{\bar{\varepsilon}}(\bar{R}, \bar{a}, \cdot)\right)^{\frac{4}{N-2}}\right) v .
$$

Therefore, we may decompose $I I$ as

$$
I I=\frac{N(N+2)}{4}\left(I I_{1}+I I_{2}\right)
$$


where

$$
\begin{gathered}
I I_{1}=\left(\left(\bar{u}_{\bar{\varepsilon}}\left(\bar{R}+\bar{S}_{2}, \bar{a}+\bar{\alpha}_{2}, x\right)\right)^{\frac{4}{N-2}}-\left(\bar{u}_{\bar{\varepsilon}}(\bar{R}, \bar{a}, x)\right)^{\frac{4}{N-2}}\right)\left(v_{2}-v_{1}\right)(x) \\
=\frac{4}{N-2}\left(v_{2}-v_{1}\right)(x) \int_{0}^{1}\left(\left(\bar{u}_{\bar{\varepsilon}}\left(\bar{R}+s \bar{S}_{2}, \bar{a}+s \bar{\alpha}_{2}, x\right)\right)^{\frac{6-N}{N-2}}\right. \\
\left.\left(\sum_{i} \mu_{i}\left(\bar{R}+s \bar{S}_{2}, \bar{a}+s \bar{\alpha}_{2}, x\right) \bar{S}_{2, i}+\sum_{i, j} \gamma_{i}^{j}\left(\bar{R}+s \bar{S}_{2}, \bar{a}+s \bar{\alpha}_{2}, x\right) \bar{\alpha}_{2, i}^{j}\right) d s\right)
\end{gathered}
$$

and

$$
\begin{aligned}
& I I_{2}=\left(\left(\bar{u}_{\bar{\varepsilon}}\left(\bar{R}+\bar{S}_{2}, \bar{a}+\bar{\alpha}_{2}, x\right)\right)^{\frac{4}{N-2}}-\left(\bar{u}_{\bar{\varepsilon}}\left(\bar{R}+\bar{S}_{1}, \bar{a}+\bar{\alpha}_{1}, x\right)\right)^{\frac{4}{N-2}}\right) v_{1}(x) \\
= & \frac{4}{N-2} v_{1}(x) \int_{0}^{1}\left(\left(\bar{u}_{\bar{\varepsilon}}\left(\bar{R}+s \bar{S}_{2}+(1-s) \bar{S}_{1}, \bar{a}+s \bar{\alpha}_{2}+(1-s) \bar{\alpha}_{1}, x\right)\right)^{\frac{6-N}{N-2}}\right. \\
& \left(\sum_{i} \mu_{i}\left(\bar{R}+s \bar{S}_{2}+(1-s) \bar{S}_{1}, \bar{a}+s \bar{\alpha}_{2}+(1-s) \bar{\alpha}_{1}, x\right)\left(\bar{S}_{2, i}-\bar{S}_{1, i}\right)\right. \\
& \left.+\sum_{i, j} \gamma_{i}^{j}\left(\bar{R}+s \bar{S}_{2}+(1-s) \bar{S}_{1}, \bar{a}+s \bar{\alpha}_{2}+(1-s) \bar{\alpha}_{1}, x\right)\left(\bar{\alpha}_{2, i}^{j}-\bar{\alpha}_{1, i}^{j}\right)\right) d s .
\end{aligned}
$$

- As before, first consider the region where $\operatorname{dist}(x, \Sigma) \leq \varepsilon^{\frac{2}{N-2}}$. As in the previous Lemma, we use the fact that there exists a constant $c>0$ only depending on $N$ such that

$$
\left|\mu_{i}(\bar{R}+\bar{S}, \bar{a}+\bar{\alpha})\right| \leq c \bar{u}_{\bar{\varepsilon}}(\bar{R}+\bar{S}, \bar{a}+\bar{\alpha}) \quad \text { and } \quad\left|\gamma_{i}^{j}\right| \leq c \operatorname{dist}(x, \Sigma) \bar{u}_{\bar{\varepsilon}}(\bar{R}+\bar{S}, \bar{a}+\bar{\alpha}) .
$$

So, we get the estimate

$$
\begin{gathered}
\left|I I_{1}\right| \leq c \operatorname{dist}(x, \Sigma)^{\nu-2}\left(\rho^{2}+\operatorname{dist}(x, \Sigma) \rho\right)\left\|v_{2}-v_{1}\right\|_{0, \alpha, \nu, 2}, \\
\left|I I_{2}\right| \leq c \varepsilon \rho^{2-\nu} \operatorname{dist}(x, \Sigma)^{\nu-2}\left(\left|\bar{S}_{2}-\bar{S}_{1}\right|+\operatorname{dist}(x, \Sigma)\left|\bar{\alpha}_{2}-\bar{\alpha}_{1}\right|\right) .
\end{gathered}
$$

- Next, assume that $\operatorname{dist}(x, \Sigma) \in\left[\varepsilon^{\frac{2}{N-2}}, 1\right]$, then as above, we can derive the estimate

$$
\begin{gathered}
\left|I I_{1}\right| \leq c \varepsilon^{\frac{4}{N-2}} \operatorname{dist}(x, \Sigma)^{\nu-4}\left(\rho^{2}+\left(\operatorname{dist}(x, \Sigma)^{N-1}+\varepsilon^{\frac{4}{N-2}} \operatorname{dist}(x, \Sigma)^{-1}\right) \rho\right)\left\|v_{2}-v_{1}\right\|_{0, \alpha, \nu, 2}, \\
\left|I I_{2}\right| \leq c \rho^{2-\nu} \varepsilon^{\frac{N+2}{N-2}} \operatorname{dist}(x, \Sigma)^{\nu-4}\left(\left|\bar{S}_{2}-\bar{S}_{1}\right|+\left(\operatorname{dist}(x, \Sigma)^{N-1}+\varepsilon^{\frac{4}{N-2}} \operatorname{dist}(x, \Sigma)^{-1}\right)\left|\bar{\alpha}_{2}-\bar{\alpha}_{1}\right|\right) .
\end{gathered}
$$

- And finally, if $\operatorname{dist}(x, \Sigma) \geq 1$, we obtain

$$
\begin{gathered}
\left|I I_{1}\right| \leq c \varepsilon^{\frac{4}{N-2}} \operatorname{dist}(x, \Sigma)^{-N-2} \rho^{2}\left\|v_{2}-v_{1}\right\|_{0, \alpha, \nu, 2}, \\
\left|I I_{2}\right| \leq c \rho^{2-\nu} \varepsilon^{\frac{N+2}{N-2}} \operatorname{dist}(x, \Sigma)^{-N-2}\left|\bar{S}_{2}-\bar{S}_{1}\right| .
\end{gathered}
$$

This allows us to conclude that

$$
\left\|I I\left(w_{1}, w_{2}\right)\right\|_{0, \alpha, \nu-2,-2} \leq c \rho^{2-\nu}\left(\rho^{2} \nu|| v_{2}-\left.v_{1}\right|_{2, \alpha, \nu, 2}+\varepsilon\left(\left|\bar{S}_{2}-\bar{S}_{1}\right|+\varepsilon^{\frac{2}{N-2}}\left|\bar{\alpha}_{2}-\bar{\alpha}_{1}\right|\right)\right),
$$

which leads to

$$
\left\|I I\left(w_{1}, w_{2}\right)\right\|_{0, \alpha, \nu-2,-2} \leq c \rho^{2-\nu}\left\|w_{2}-w_{1}\right\|_{\tilde{\mathcal{M}}} .
$$

Then the estimate of the Lemma follows from the result of Proposition 9.

We now turn to the estimate of the image by $\tilde{\Lambda}_{\bar{\varepsilon}, \bar{R}, \bar{a}}^{-1}$ of

$$
I I I\left(w_{1}, w_{2}\right) \equiv\left(D \mathcal{N}_{\mid\left(0, \bar{S}_{2}, \bar{\alpha}_{2}\right)}-D \mathcal{N}_{\mid(0,0,0)}\right)\left(0, \bar{S}_{2}, \bar{\alpha}_{2}\right)-\left(D \mathcal{N}_{\mid\left(0, \bar{S}_{1}, \bar{\alpha}_{1}\right)}-D \mathcal{N}_{\mid(0,0,0)}\right)\left(0, \bar{S}_{1}, \bar{\alpha}_{1}\right) .
$$

As before we decompose $I I I=I I I_{1}+I I I_{2}$ where

$$
I I I_{1} \equiv\left(D \mathcal{N}_{\mid\left(0, \bar{S}_{2}, \bar{\alpha}_{2}\right)}-D \mathcal{N}_{\mid(0,0,0)}\right)\left(0, \bar{S}_{2}-\bar{S}_{1}, \bar{\alpha}_{2}-\bar{\alpha}_{1}\right)
$$




$$
I I I_{2}=\left(D \mathcal{N}_{\mid\left(0, \bar{S}_{2}, \bar{\alpha}_{2}\right)}-D \mathcal{N}_{\mid\left(0, \bar{S}_{1}, \bar{\alpha}_{1}\right)}\right)\left(0, \bar{S}_{1}, \bar{\alpha}_{1}\right)
$$

To estimate either of these terms, it is convenient to consider them as special cases of the more general quantity

$$
\overline{I I I}\left(\bar{S}, \bar{S}^{\prime}, \bar{S}^{\prime \prime}, \bar{\alpha}, \bar{\alpha}^{\prime}, \bar{\alpha}^{\prime \prime}\right)=\left(D \mathcal{N}_{\mid\left(0, \bar{S}+\bar{S}^{\prime}, \bar{\alpha}+\bar{\alpha}^{\prime}\right)}-D \mathcal{N}_{\mid(0, \bar{S}, \bar{\alpha})}\right)\left(0, \bar{S}^{\prime \prime}, \bar{\alpha}^{\prime \prime}\right)
$$

We first prove the Lemma :

Lemma 13 There exists some constant $c>0$ (depending on $C_{0}$ ) such that

$$
\|\left.\tilde{\Lambda}_{\bar{\varepsilon}, \bar{R}, \bar{a}}^{-1} \overline{I I I}\left(\bar{S}, \bar{S}^{\prime}, \bar{S}^{\prime \prime}, \bar{\alpha}, \bar{\alpha}^{\prime}, \bar{\alpha}^{\prime \prime}\right)\right|_{\tilde{\mathcal{M}}} \leq c \varepsilon\left(\left|\bar{S}^{\prime}\right|\left|\bar{S}^{\prime \prime}\right|+\rho^{2}\left|\bar{\alpha}^{\prime}\right|\left|\bar{\alpha}^{\prime \prime}\right|+\rho\left|\bar{S}^{\prime}\right|\left|\bar{\alpha}^{\prime \prime}\right|+\rho\left|\bar{\alpha}^{\prime}\right|\left|\bar{S}^{\prime \prime}\right|\right) .
$$

Proof : We may write

$$
\overline{I I I}\left(\bar{S}, \bar{S}^{\prime}, \bar{S}^{\prime \prime}, \bar{\alpha}, \bar{\alpha}^{\prime}, \bar{\alpha}^{\prime \prime}\right)=\int_{0}^{1} D^{2} \mathcal{N}_{\mid\left(0, \bar{S}+s \bar{S}^{\prime}, \bar{\alpha}+s \bar{\alpha}^{\prime}\right)}\left(\left(0, \bar{S}^{\prime}, \bar{\alpha}^{\prime}\right),\left(0, \bar{S}^{\prime \prime}, \bar{\alpha}^{\prime \prime}\right)\right) d s,
$$

and shall concentrate on estimating integrands of the form

$$
\overline{i i i}\left(\bar{S}, \bar{S}^{\prime}, \bar{S}^{\prime \prime}, \bar{\alpha}, \bar{\alpha}^{\prime}, \bar{\alpha}^{\prime \prime}\right) \equiv D^{2} \mathcal{N}_{\mid(0, \bar{S}, \bar{\alpha})}\left(\left(0, \bar{S}^{\prime}, \bar{\alpha}^{\prime}\right),\left(0, \bar{S}^{\prime \prime}, \bar{\alpha}^{\prime \prime}\right)\right) .
$$

We take advantage from the observation that, for all $\bar{R} \in \mathbb{R}^{n}$ and for all $\bar{a} \in\left(\mathbb{R}^{N}\right)^{n}$

$$
\Delta \bar{u}_{\bar{\varepsilon}}(\bar{R}, \bar{a}, x)+\frac{N(N-2)}{4}\left(\bar{u}_{\bar{\varepsilon}}(\bar{R}, \bar{a}, x)\right)^{\frac{N+2}{N-2}}=0,
$$

if $\operatorname{dist}\left(x, x_{i}\right) \leq \rho_{i}$. Therefore, if $\operatorname{dist}\left(x, x_{i}\right) \leq \rho_{i},(79)$ is identically 0 . Moreover, if $\operatorname{dist}\left(x, x_{i}\right) \geq 2 \rho_{i}$, we observe that $\bar{u}_{\bar{\varepsilon}}(\bar{R}, \bar{a}, x)=\bar{w}_{\bar{\varepsilon}}(\bar{R}, x)$ is harmonic and does not depend on $\bar{a}$. Therefore, in this case, $\overline{i i i}$ reduces to

$$
\overline{i i i}=\frac{N(N-2)}{4} \sum_{i, j} \partial_{R_{i}, R_{j}}^{2}\left(\left(\bar{w}_{\bar{\varepsilon}}(\bar{R}+\bar{S})^{\frac{N+2}{N-2}}\right) S_{i}^{\prime} S_{j}^{\prime \prime},\right.
$$

And, still assuming that $\operatorname{dist}\left(x, x_{i}\right) \geq 2 \rho_{i}$, we find the estimate

$$
|\overline{i i i}| \leq c \varepsilon^{\frac{N+2}{N-2}} \operatorname{dist}(x, \Sigma)^{-N-2}\left|\bar{S}^{\prime}\right|\left|\bar{S}^{\prime \prime}\right| .
$$

Finally, if $\operatorname{dist}\left(x, x_{i}\right) \in\left[\rho_{i}, 2 \rho_{i}\right]$, then by definition

$$
\bar{u}_{\bar{\varepsilon}}(\bar{R}, \bar{a}, x)=\chi_{i}\left(x-x_{i}\right) u_{\varepsilon_{i}}\left(R_{i}, a_{i}, x-x_{i}\right)+\bar{w}_{\bar{\varepsilon}}(\bar{R}, x)\left(1-\chi_{i}\left(x-x_{i}\right)\right),
$$

so we can write

$$
\begin{gathered}
\Delta \bar{u}_{\bar{\varepsilon}}(\bar{R}, \bar{a}, x)+\frac{N(N-2)}{4}\left(\bar{u}_{\bar{\varepsilon}}(\bar{R}, \bar{a}, x)\right)^{\frac{N+2}{N-2}} \\
=\frac{N(N-2)}{4}\left(-\chi_{i}\left(u_{\varepsilon_{i}}\left(R_{i}, a_{i}, \cdot-x_{i}\right)\right)^{\frac{N+2}{N-2}}+\left(\chi_{i} u_{\varepsilon_{i}}\left(R_{i}, a_{i}, \cdot-x_{i}\right)+\left(1-\chi_{i}\right) \bar{w}_{\bar{\varepsilon}}(\bar{R}, \cdot)\right)^{\frac{N+2}{N-2}}\right) \\
+\Delta\left(\chi_{i}\right)\left(u_{\varepsilon_{i}}\left(R_{i}, a_{i}, \cdot-x_{i}\right)-\bar{w}_{\bar{\varepsilon}}(\bar{R}, \cdot)\right)+2 \nabla\left(\chi_{i}\right) \nabla\left(u_{\varepsilon_{i}}\left(R_{i}, a_{i}, \cdot-x_{i}\right)-\bar{w}_{\bar{\varepsilon}}(\bar{R}, \cdot)\right) .
\end{gathered}
$$

Differentiating twice with respect to both $\bar{R}$ and $\bar{a}$, it is easy to derive the estimate

$$
\begin{gathered}
\left|D_{\mid(\bar{R}+\bar{S}, \bar{a}+\bar{\alpha})}^{2}\left(-\chi_{i}\left(u_{\varepsilon_{i}}\right)^{\frac{N+2}{N-2}}+\left(\chi_{i} u_{\varepsilon_{i}}+\left(1-\chi_{i}\right) \bar{w}_{\bar{\varepsilon}}\right)^{\frac{N+2}{N-2}}\right)\left(\bar{S}^{\prime}, \bar{\alpha}^{\prime}\right)\left(\bar{S}^{\prime \prime}, \bar{\alpha}^{\prime \prime}\right)\right| \\
\leq c \varepsilon^{\frac{N+2}{N-2}}\left(\rho^{-4}\left(\rho^{2-N}\left|\bar{S}^{\prime}\right|\left|\bar{S}^{\prime \prime}\right|+\rho\left(\left|\alpha^{\prime}\right|\left|\bar{S}^{\prime \prime}\right|+\left|\alpha^{\prime \prime}\right|\left|\bar{S}^{\prime}\right|\right)+\rho^{2}\left|\alpha^{\prime}\right|\left|\alpha^{\prime \prime}\right|\right)\right. \\
\left.\quad+\rho^{N-6}\left(\rho^{2-N}\left|\bar{S}^{\prime}\right|+\rho\left|\bar{\alpha}^{\prime}\right|\right)\left(\rho^{2-N}\left|\bar{S}^{\prime \prime}\right|+\rho\left|\bar{\alpha}^{\prime \prime}\right|\right)\right) \\
\leq c \varepsilon^{\frac{N+2}{N-2}}\left(\rho^{-2-N}\left|\bar{S}^{\prime}\right|\left|\bar{S}^{\prime \prime}\right|+\rho^{-3}\left(\left|\alpha^{\prime}\right|\left|\bar{S}^{\prime \prime}\right|+\left|\alpha^{\prime \prime}\right|\left|\bar{S}^{\prime}\right|\right)+\rho^{-2}\left|\alpha^{\prime}\right|\left|\alpha^{\prime \prime}\right|\right) .
\end{gathered}
$$


Using the result of Proposition 4 and differentiating twice with respect to both $\bar{R}$ and $\bar{a}$, we get the expansion

$$
\begin{gathered}
D_{\mid(\bar{R}+\bar{S}, \bar{a}+\bar{\alpha})}^{2}\left(u_{\varepsilon_{i_{0}}}-\bar{w}_{\bar{\varepsilon}}(\bar{R}, x)\right)\left(\bar{S}^{\prime}, \bar{\alpha}^{\prime}\right)\left(\bar{S}^{\prime \prime}, \bar{\alpha}^{\prime \prime}\right) \\
=D_{\mid(\bar{R}+\bar{S}, \bar{a}+\bar{\alpha})}^{2}\left(\frac{\varepsilon_{i_{0}}}{2} R_{i_{0}}^{\frac{2-N}{2}}-\sum_{i \neq i_{0}} \frac{\varepsilon_{i}}{2} R_{i}^{\frac{N-2}{2}}\left|x_{i_{0}}-x_{i}\right|^{2-N}\right)\left(\bar{S}^{\prime}, \bar{\alpha}^{\prime}\right)\left(\bar{S}^{\prime \prime}, \bar{\alpha}^{\prime \prime}\right) \\
+\frac{N-2}{2} D_{\mid(\bar{R}+\bar{S}, \bar{a}+\bar{\alpha})}^{2}\left(\left(\varepsilon_{i_{0}} R_{i_{0}}^{\frac{2-N}{2}} a_{i_{0}}+\sum_{i \neq i_{0}} \frac{\varepsilon_{i}}{2} R_{i}^{\frac{N-2}{2}}\left|x_{i_{0}}-x_{i}\right|^{-N}\left(x_{i_{0}}-x_{i}\right)\right) \cdot\left(x-x_{i_{0}}\right)\right)\left(\bar{S}^{\prime}, \bar{\alpha}^{\prime}\right)\left(\bar{S}^{\prime \prime}, \bar{\alpha}^{\prime \prime}\right) \\
+O\left(\varepsilon \rho^{2}\right)\left(\left|\bar{S}^{\prime}\right|\left|\bar{S}^{\prime \prime}\right|+\left|\bar{\alpha}^{\prime}\right|\left|\bar{\alpha}^{\prime \prime}\right|+\left|\bar{S}^{\prime}\right|\left|\bar{\alpha}^{\prime \prime}\right|+\left|\bar{\alpha}^{\prime}\right|\left|\bar{S}^{\prime \prime}\right|\right) .
\end{gathered}
$$

and

$$
\begin{gathered}
D_{\mid(\bar{R}+\bar{S}, \bar{a}+\bar{\alpha})}^{2}\left(\partial_{r_{i_{0}}}\left(u_{\varepsilon_{i_{0}}}\left(R_{i_{0}}, a_{i_{0}}, x-x_{i_{0}}\right)-\bar{w}_{\bar{\varepsilon}}(\bar{R}, x)\right)\right)\left(\bar{S}^{\prime}, \bar{\alpha}^{\prime}\right)\left(\bar{S}^{\prime \prime}, \bar{\alpha}^{\prime \prime}\right) \\
=\frac{N-2}{2} D_{\mid(\bar{R}+\bar{S}, \bar{a}+\bar{\alpha})}^{2}\left(\left(\varepsilon_{i_{0}} R_{i_{0}}^{\frac{2-N}{2}} a_{i_{0}}-\sum_{i \neq i_{0}} \frac{\varepsilon_{i}}{2} R_{i}^{\frac{N-2}{2}}\left|x_{i_{0}}-x_{i}\right|^{-N}\left(x_{i_{0}}-x_{i}\right)\right) \cdot \frac{x-x_{i_{0}}}{\left|x-x_{i_{0}}\right|}\right)\left(\bar{S}^{\prime}, \bar{\alpha}^{\prime}\right)\left(\bar{S}^{\prime \prime}, \bar{\alpha}^{\prime \prime}\right) \\
+O(\varepsilon \rho)\left(\left|\bar{S}^{\prime}\right|\left|\bar{S}^{\prime \prime}\right|+\left|\bar{\alpha}^{\prime}\right|\left|\bar{\alpha}^{\prime \prime}\right|+\left|\bar{S}^{\prime}\right|\left|\bar{\alpha}^{\prime \prime}\right|+\left|\bar{\alpha}^{\prime}\right|\left|\bar{S}^{\prime \prime}\right|\right) .
\end{gathered}
$$

This expansion allows us to estimate

$$
\begin{gathered}
D_{\mid(\bar{R}+\bar{S}, \bar{a}+\bar{\alpha})}^{2}\left(\Delta\left(\chi_{i}\right)\left(u_{\varepsilon_{i}}\left(R_{i}, a_{i}, \cdot-x_{i}\right)-\bar{w}_{\bar{\varepsilon}}(\bar{R}, \cdot)\right)+\right. \\
\left.2 \nabla\left(\chi_{i}\right) \nabla\left(u_{\varepsilon_{i}}\left(R_{i}, a_{i}, \cdot-x_{i}\right)-\bar{w}_{\bar{\varepsilon}}(\bar{R}, \cdot)\right)\right)\left(\bar{S}^{\prime}, \bar{\alpha}^{\prime}\right)\left(\bar{S}^{\prime \prime}, \bar{\alpha}^{\prime \prime}\right) .
\end{gathered}
$$

As in the setting of Lemma 9 , we shall decompose this quantity as $h_{0}+h^{\prime}+h^{\prime \prime}$ as before, then we get the estimates

$$
\begin{gathered}
\left|h_{0}\right| \leq c \varepsilon\left(\rho^{-2}\left|\bar{S}^{\prime}\right|\left|\bar{S}^{\prime \prime}\right|+\left|\bar{\alpha}^{\prime}\right|\left|\bar{\alpha}^{\prime \prime}\right|+\left|\bar{S}^{\prime}\right|\left|\bar{\alpha}^{\prime \prime}\right|+\left|\bar{\alpha}^{\prime}\right|\left|\bar{S}^{\prime \prime}\right|\right) . \\
\left|h^{\prime}\right| \leq c \varepsilon\left(\rho^{-1}\left|\bar{S}^{\prime}\right|\left|\bar{S}^{\prime \prime}\right|+\left|\bar{\alpha}^{\prime}\right|\left|\bar{\alpha}^{\prime \prime}\right|+\rho^{-1}\left|\bar{S}^{\prime}\right|\left|\bar{\alpha}^{\prime \prime}\right|+\rho^{-1}\left|\bar{\alpha}^{\prime}\right|\left|\bar{S}^{\prime \prime}\right|\right) .
\end{gathered}
$$

and finally

$$
\left|h^{\prime \prime}\right| \leq c \varepsilon\left(\left|\bar{S}^{\prime}\right|\left|\bar{S}^{\prime \prime}\right|+\left|\bar{\alpha}^{\prime}\right|\left|\bar{\alpha}^{\prime \prime}\right|+\left|\bar{S}^{\prime}\right|\left|\bar{\alpha}^{\prime \prime}\right|+\left|\bar{\alpha}^{\prime}\right|\left|\bar{S}^{\prime \prime}\right|\right) .
$$

Therefore, using the result of Proposition 9 as well as the result of Lemma 9, with $\mu=0$, we obtain

$$
\|\left.\tilde{\Lambda}_{\bar{\varepsilon}, \bar{R}, \bar{a}}^{-1} \overline{i i i}\left(\bar{S}, \bar{S}^{\prime}, \bar{S}^{\prime \prime}, \bar{\alpha}, \bar{\alpha}^{\prime}, \bar{\alpha}^{\prime \prime}\right)\right|_{\tilde{\mathcal{M}}} \leq c \varepsilon\left(\left|\bar{S}^{\prime}\right|\left|\bar{S}^{\prime \prime}\right|+\rho^{2}\left|\bar{\alpha}^{\prime}\right|\left|\bar{\alpha}^{\prime \prime}\right|+\rho\left|\bar{S}^{\prime}\right|\left|\bar{\alpha}^{\prime \prime}\right|+\rho\left|\bar{\alpha}^{\prime}\right|\left|\bar{S}^{\prime \prime}\right|\right) .
$$

As a consequence we get that

$$
\|\left.\tilde{\Lambda}_{\bar{\varepsilon}, \bar{R}, \bar{a}}^{-1} \overline{I I I}\left(\bar{S}, \bar{S}^{\prime}, \bar{S}^{\prime \prime}, \bar{\alpha}, \bar{\alpha}^{\prime}, \bar{\alpha}^{\prime \prime}\right)\right|_{\tilde{\mathcal{M}}} \leq c \varepsilon\left(\left|\bar{S}^{\prime}\right|\left|\bar{S}^{\prime \prime}\right|+\rho^{2}\left|\bar{\alpha}^{\prime}\right|\left|\bar{\alpha}^{\prime \prime}\right|+\rho\left|\bar{S}^{\prime}\right|\left|\bar{\alpha}^{\prime \prime}\right|+\rho\left|\bar{\alpha}^{\prime}\right|\left|\bar{S}^{\prime \prime}\right|\right) .
$$

As a corollary of this last lemma, recalling that $\varepsilon\left|\bar{S}_{j}\right|+\varepsilon \rho\left|\bar{\alpha}_{j}\right| \leq C_{0} \varepsilon \rho^{2}$, we get that

$$
\left\|\left.\tilde{\Lambda}_{\bar{\varepsilon}, \bar{R}, \bar{a}}^{-1} I I I\left(w_{1}, w_{2}\right)\right|_{\tilde{\mathcal{M}}} \leq c \varepsilon\left(\rho^{2}\left|\bar{S}_{2}-\bar{S}_{1}^{\prime}\right|+\rho^{3}\left|\bar{\alpha}_{2}-\bar{\alpha}_{1}\right|\right) \leq c \rho^{2}|| w_{2}-w_{1}\right\|_{\tilde{\mathcal{M}}}
$$

Collecting the results of these lemmata, we have established that for some constant $c>0$ depending on $C_{0}$,

$$
\left\|\mathcal{P}\left(w_{2}\right)-\mathcal{P}\left(w_{1}\right)\right\|_{\tilde{\mathcal{M}}} \leq c \rho^{2-\nu}\left\|w_{2}-w_{1}\right\|_{\tilde{\mathcal{M}}}
$$

By taking $\varepsilon$, and hence $\rho$, sufficiently small, we have shown that the map $\mathcal{P}$ is a contraction on the ball of radius $C_{0} \varepsilon \rho^{2}$ in $\tilde{\mathcal{M}}$. Hence it has a unique fixed point $w$, and this function $w=(v, \bar{S}, \bar{\alpha})$ is a solution of the equation (77). It is clear that $\bar{u}_{\bar{\varepsilon}}(\bar{R}+\bar{S}, \bar{a}+\bar{\alpha}, \cdot)+v$ is positive near the points of $\Sigma$, hence by the maximum principle is positive everywhere.

This completes the existence of the solution promised in Theorem 1. 


\section{The nondegeneracy of the solutions}

We now show that for $\varepsilon$ sufficiently small, the solutions we have constructed above are nondegenerate in the sense defined in [7], [8]. Actually, there are two closely related notions of nondegeneracy, the definitions of which we now recall, in terms of the notations of this paper:

Definition 3 Let $g_{0}$ be the standard metric on $S^{N}$. A metric $g=u^{\frac{4}{N-2}} g_{0}$ of constant positive scalar curvature on $S^{N} \backslash \Lambda$, as well as the corresponding conformal factor $u$, is called marked nondegenerate if the linearization of the scalar curvature operator $\mathcal{N}$ for $g_{0}$ about the solution $u$ is injective on the function space $\mathcal{C}_{\mu}^{2, \alpha}\left(S^{N} \backslash \Lambda\right)$ for all $\mu>(2-N) / 2$, or equivalently, if the linearization of $\mathcal{N}$ relative to the metric $g$ about the constant solution 1 is injective on the function space $\mathcal{C}_{\delta}^{2, \alpha}\left(S^{N} \backslash \Lambda\right)$ (defined with respect to the metric $g$ ) for all $\delta>0$. The metric $g$, or solution $u$, is called unmarked nondegenerate if the linearization is injective for all $\mu>(4-N) / 2$, or equivalently, for all $\delta>1$.

These two nondegeneracy conditions are precisely what is needed to ensure the smoothness of the marked and unmarked moduli spaces $\mathcal{M}_{\Lambda}$ and $\mathcal{M}_{n}$ at $g$. The former of these spaces is the space of all metrics of constant positive scalar curvature on the complement of the finite set $\Lambda$ in the sphere $S^{N}$, while the latter is the set of all such metrics on the complement of any finite set $\tilde{\Lambda}$ of cardinality $n$. As proved in [7] and [8], these moduli spaces are real analytic sets, hence are stratified and may be written as the union of smooth, real analytic manifolds of varying dimensions. The existence of one smooth point in a given component shows that the top dimensional stratum in that component is of the dimension predicted by the formal, index-theoretic, calculations, namely $n$ for the marked spaces and $n(N+1)$ for the unmarked ones.

In this section we shall prove

Proposition 10 The solutions constructed here are unmarked nondegenerate. For generic configurations $\Lambda$ they are also marked nondegenerate.

As a corollary, we obtain smoothness of the unmarked moduli spaces without any restriction (when $\varepsilon$ is small) and of the marked moduli spaces for generic configurations. In fact, near a generic $\Lambda$, and when $\varepsilon$ is sufficiently small, we may use $\left(p_{1}, \ldots, p_{n}, \varepsilon_{1}, \ldots, \varepsilon_{n}\right)=(\bar{p}, \bar{\varepsilon}) \in$ $\left(S^{N}\right)^{n} \times\left(\mathbb{R}^{+}\right)^{n}$ as coordinates on the unmarked moduli space $\mathcal{M}_{n}$, and $\bar{\varepsilon}$ as coordinates on the marked moduli spaces. We note that marked nondegenerate solutions (which are a fortiori also unmarked nondegenerate) were constructed in [8] for certain very special configurations $\Lambda$, which in particular contain only even numbers of points; the Delaunay parameters of those solutions are not precisely prescribed, but they need not be close to zero. Even when $n$ is even, it is not clear that these solutions lie in the same component of $\mathcal{M}_{n}$ as the ones we construct above.

We first demonstrate the unmarked nondegeneracy; the proof is by contradiction. By a slight change of notation from the rest of the paper, we consider the solution $u$ on $S^{N} \backslash \Lambda$ to have the form $u=\bar{u}_{\bar{\varepsilon}}(\bar{R}, \bar{a}, \cdot)+v$, where the approximate solution on the sphere (rather than on $\mathbb{R}^{N}$ ) is now denoted $\bar{u}_{\bar{\varepsilon}}(\bar{R}, \bar{a}, \cdot)$, and $v$ is an element of $\mathcal{C}_{\nu}^{2, \alpha}$ with $1<\nu<2$, with norm in this space bounded by $C_{0} \varepsilon \rho^{2-\nu}$. Assume that, for some sequence of $\varepsilon_{k}$ tending to 0 , the linearized operator

$$
\mathcal{L}_{k}=\Delta_{S^{N}}-\frac{N(N-2)}{4}+\frac{N(N+2)}{4} u_{k}^{\frac{4}{N-2}}
$$

is not injective on $\mathcal{C}_{\mu}^{2, \alpha}\left(S^{N} \backslash \Lambda\right)$ for some $\mu>(4-N) / 2$. Here we have denoted by $u_{k}$ the solution $\bar{u}_{\bar{\varepsilon}_{k}}\left(\bar{R}_{k}, \bar{a}_{k}, \cdot\right)+v_{k}$. Then there is some element $w_{k} \in \mathcal{C}_{\mu}^{2, \alpha}$ such that $\mathcal{L}_{k} w_{k}=0$.

First normalize $w_{k}$, multiplying it by a suitable constant, so that $\sup d(y)^{-\mu}\left|w_{k}(y)\right|=1$, where $d(y)$ is the distance of the point $y$ from $\Lambda$, say in the spherical metric. Choose a point $y_{k} \in S^{N} \backslash \Lambda$ realizing this supremum, i.e. such that $d\left(y_{k}\right)^{-\mu}\left|w_{k}\left(y_{k}\right)\right|=1$. As $k$ tends to infinity, the function $v_{k}$ tends to zero in $\mathcal{C}_{\nu}^{2, \alpha}$, since its norm is dominated by $C_{0} \varepsilon_{k} \rho_{k}^{2-\nu}$, and $\bar{u}_{\bar{\varepsilon}_{k}}$ converges uniformly 
to zero on compact subsets of $S^{N} \backslash \Lambda$. In addition, we can assume that the sequences $\bar{R}_{k}$ and $\bar{a}_{k}$ converge to some fixed $\bar{R}^{*}$ and $\bar{a}^{*}$, respectively.

Suppose first that some subsequence of the $y_{k}$ converges to a point $y_{0} \in S^{N} \backslash \Lambda$. Then we can extract a subsequence of the $w_{k}$ which converge (in $\mathcal{C}^{\infty}$ ) to a limiting function $w$ on $S^{N} \backslash \Lambda$; $w$ must be nontrivial since $d\left(y_{0}\right)^{-\mu}\left|w\left(y_{0}\right)\right|=1$. Furthermore, $L w \equiv\left(\Delta_{S^{N}}-(N-2)^{2} / 4\right) w=0$, and $|w(y)| \leq d(y)^{\mu}$. Since $\mu>(4-N) / 2$, it is standard that $w$ is a weak solution of $L w=0$ on all of $S^{N}$, hence also a strong solution. But clearly $L$ has only trivial nullspace, hence $w \equiv 0$, which is a contradiction.

If, on the other hand, some subsequence of the $y_{k}$ converge to one of the points $p_{i_{0}} \in \Lambda$, then we must argue somewhat differently. Choose a function $A$ on $S^{N} \backslash\left\{p_{i_{0}}, q\right\}$, where $q \notin \Lambda$, which transforms this twice-punctured sphere to the cylinder $\mathbb{R} \times S^{N-1}$ and such that the standard spherical metric $g_{S}$ and the (dilated) cylindrical metric $g_{C}$ are related by

$$
g_{S}=A^{\frac{4}{N-2}} g_{C}, \quad g_{C}=\frac{N-2}{N}\left(d t^{2}+d \theta^{2}\right) .
$$

On $C$, the function $A$ is simply a multiple of $(\cosh t)^{\frac{2-N}{2}}$, and on $S^{N}$ is of the order $d(y, p)^{\frac{2-N}{2}}$, for $p=p_{i_{0}}$ or $q$. Noting that $g_{C}$ has scalar curvature $N(N-1)$, the same as $g_{S}$, the conformal Laplacians of these two metrics, $L_{C}$ and $L_{S}$ satisfy the usual transformation rule

$$
L_{C}(A \phi)=A^{\frac{N+2}{N-2}} L_{S} \phi
$$

for any function $\phi$. The solution $u_{k}$ on $S^{N}$ corresponds to a solution $A u_{k}$ on $C$. It is easy to check that the linearizations $\mathcal{L}_{S, k}$ of the scalar curvature operators on $S^{N}$ at $u_{k}$ and $\mathcal{L}_{C, k}$ on $C$ at $A u_{k}$ satisfy the same transformation rule (82) as $L_{S}$ and $L_{C}$, cf. [8]. (Unlike the transformation rule for the conformal Laplacian, this holds only because we are dealing with two metrics of the same constant scalar curvature.)

Because of these machinations, we may replace $w_{k}$ by a solution $\tilde{w}_{k}$ of $\mathcal{L}_{C, k} \tilde{w}_{k}=0$. For convenience, we call $\mathcal{L}_{C, k}$ simply $\mathcal{L}_{k}$ and $\tilde{w}_{k}$ simply $w_{k}$ again, and let $y=(t, \theta)$ denote the variable on the cylinder. Define $\delta=\mu+(N-2) / 2$, so that $\delta>1$. These new functions $w_{k}$ satisfy

$$
\sup d(y)^{-\delta}\left|w_{k}\right|=1 \text {, }
$$

where $d(y)$ is some function equalling the distance to the set of other singular points $\Lambda^{\prime}=\Lambda \backslash\left\{p_{i_{0}}\right\}$ (transplanted to the cylinder) in a neighbourhood of this set, and equalling $e^{-t}$ outside this neighbourhood. Because of $(83), w_{k}$ decays at either end of the cylinder.

As before, let $y_{k}=\left(t_{k}, \theta_{k}\right)$ denote the point on $C$ where the supremum in (83) is attained. We already are assuming that $t_{k} \rightarrow \infty$. By translating back by $t_{k}$ and multiplying by a suitable constant, we find yet another sequence of solutions, which we again call $w_{k}$, attaining their maximum at $t=0$, and which solve the translated equation, which we again write as $\mathcal{L}_{k} w_{k}=0$. Here $\mathcal{L}_{k}$ is the linearized scalar curvature operator relative to a metric $g_{k}=\left(A\left(\bar{u}_{\bar{\varepsilon}_{k}}+v_{k}\right)\right)^{4 /(N-2)} g_{C}$ which is singular at a finite collection of points which are translating toward $t=-\infty$. Since $v_{k}$ is tending to zero, $g_{k}$ more and more nearly approximates the Delaunay metric $V_{\varepsilon_{k}}^{4 /(N-2)} g_{C}$, where $V_{\varepsilon_{k}}$ is one of the ( $\theta$-independent) Delaunay solutions considered in $\S 2$.

It is not hard to see that the $w_{k}$ converge to a nontrivial solution $w$ of the limiting equation $\mathcal{L} w=0$, and that $w$ is bounded by $e^{-\delta t}$ for all $t$.

There are two subcases. In the first, $V_{k}(0, \theta)$ tends to zero. Then $w$ satisfies the equation

$$
\frac{N}{N-2}\left(\partial_{t}^{2}+\Delta_{S^{N-1}}\right) w-\frac{N(N-2)}{4} w=\frac{N}{N-2}\left(\partial_{t}^{2}+\Delta_{S^{N-1}}-\frac{(N-2)^{2}}{4}\right) w=0 .
$$

By decomposing $w$ into eigencomponents with respect to $\Delta_{S^{N-1}}$, we see that any eigencomponent $w_{j}$ is a sum of exponentials, $w_{j}=a_{j}^{+} e^{\gamma_{j} t}+a_{j}^{-} e^{-\gamma_{j} t}$. Since $w$ decays as $t \rightarrow+\infty, a_{j}^{+}=0$. But then it is clear that no function of the form $e^{-\gamma_{j} t}$ can be bounded for all $t$ by $e^{-\delta t}$ unless $\delta=\gamma_{j}$, 
which is not the case, so we arrive at a contradiction. In the second, $V_{k}(0, \theta)$ does not tend to zero. By translating by a fixed finite amount, we may assume that $V_{k}$ tends to the function $(\cosh t)^{(2-N) / 2}$, and hence, after pulling out the superfluous constants, that the limiting function $w$ satisfies

$$
\left(\partial_{t}^{2}+\Delta_{S^{N-1}}-\frac{(N-2)^{2}}{4}+\frac{N^{2}-4}{4} \operatorname{sech}^{2} t\right) w=0 .
$$

Again separate $w$ into its eigencomponents $w_{j}$. Then

$$
\partial_{t}^{2} w_{j}-\left(\frac{(N-2)^{2}}{4}+\lambda_{j}\right) w_{j}+\frac{N^{2}-4}{4} \operatorname{sech}^{2} t w_{j}=0
$$

For $j=0$ the indicial roots of this equation at both $\pm \infty$ are $\pm(N-2) / 2$, for $j=1, \ldots, N$ they are $\pm N / 2$, and for $j>N$ they all satisfy $\left|\gamma_{j}^{ \pm}\right| \geq(N+2) / 2$.

The components $w_{j}$ with $j>N$ are easy to eliminate. In fact, these $w_{j}$ must decay faster than $e^{ \pm(N+2)|t| / 2}$ at $\pm \infty$, so we may multiply the equation satisfied by $w_{j}$ and integrate by parts to obtain

$$
\int_{-\infty}^{\infty}\left(\partial_{t} w_{j}\right)^{2}+\left(\lambda_{j}+\frac{(N-2)^{2}}{4}\right) w_{j}^{2}-\frac{N^{2}-4}{4} \operatorname{sech}^{2} t w_{j}^{2} d t=0 .
$$

Since $\lambda_{j} \geq 2 N$, the integrand is nonnegative, hence $w_{j}=0$.

For the remaining cases, when $j \leq N$, the indicial roots at $\pm \infty$ are less than $(N+2) / 2$ in absolute value. On the other hand, to check unmarked nondegeneracy it suffices to use any $\delta>1$ which is also less than the next omitted weight for the linearization about a Delaunay solution, as determined in $\S 5$. But by the results of that section, as $\varepsilon$ tends to zero, this next omitted weight tends to $(N+2) / 2$. Thus now choose $\delta$ in the range $(N / 2,(N+2) / 2)$; then each of the $w_{j}$ with $j \leq N$ decays less quickly than $e^{-\delta t}$ as $t \rightarrow+\infty$, which implies that these $w_{j}$ too must vanish. This is a contradiction.

This covers all cases, so we have showed that the linearization is injective on the appropriate weighted Hölder spaces.

This completes the proof of unmarked nondegeneracy of the solutions when $\varepsilon$ is sufficiently small. We complete the proof of the proposition by demonstrating the marked nondegeneracy of solutions for generic configurations $\Lambda$. This is a simple consequence of Sard's theorem. In fact, as discussed in [8], near smooth points of the unmarked moduli space $\mathcal{M}_{n}$, there is a real analytic fibration $\pi: \mathcal{M}_{n} \rightarrow \mathcal{C}_{n}$ onto the configuration space of $n$ distinct points in $S^{N}$. $\mathcal{C}_{n}$ is itself a real analytic manifold. Standard differential topological arguments now imply the surjectivity of the differential $\pi_{*}$ for all points in a generic fibre of $\pi$. By a dimension count, surjectivity of this differential is equivalent to the marked nondegeneracy of all points in the fibre.

\section{The general case}

In this brief final section we discuss the essentially minor changes that need to be made in order to prove the more general statement of Theorem 1, where the singular set is allowed to have components of positive dimension. As in the main body of the paper, there are three steps. First, we must construct an approximate solution, or rather, a family of approximate solutions that become increasingly concentrated at $\Lambda$; next we must prove that the linearization about one of these approximate solutions is surjective on an appropriate function space provided the approximate solutions are sufficiently concentrated; finally, we perturb once again to an exact solution. In each of these steps, we must somehow combine the constructions and proofs from our previous paper [6] with the ones here. The last step, showing that an appropriate map is a contraction, is straightforward and we shall not comment on it further. We now describe the first two steps.

As in the introduction, divide the components of the singular set $\Lambda$ into two groups, $\Lambda=$ $\Lambda^{\prime} \cup \Lambda^{\prime \prime}$, where the first is a finite set and the latter contains all the higher dimensional components. 
The construction of a family of approximate solutions around a component $\Lambda_{j}$ of dimension $k$, where $0<k \leq(N-2) / 2$, is given in detail in [6], but briefly, it is obtained by first fixing a tubular neighbourhood $\mathcal{T}\left(\Lambda_{j}\right)$ and identifying it with a neighbourhood of the zero section in the normal bundle $N \Lambda_{j}$. On each of the fibres $N_{p} \Lambda_{j}$ we glue in a sufficiently dilated solution of the equation

$$
\Delta u+u^{\frac{N+2}{N-2}}=0
$$

cut off to be supported in $\mathcal{T}\left(\Lambda_{j}\right)$. Because the fibres are of dimension $N-k$, this equation is subcritical, and one can show that the radial solutions have the form $|x|^{(2-N) / 2} v$, where $v$ tends to zero rather quickly as $|x| \rightarrow \infty$. There is a one-parameter family $u_{\varepsilon}$ of such solutions, which are all, in a suitable sense, dilated versions of one another. Again, we refer to [6] for all details.

To construct the approximate solutions when $\Lambda^{\prime}$ and $\Lambda^{\prime \prime}$ are both nonempty, we fix Delaunay parameters $\bar{\varepsilon}^{\prime}$ for the points of $\Lambda^{\prime}$ and dilation parameters $\bar{\varepsilon}^{\prime \prime}$ for the components of $\Lambda^{\prime \prime}$, and set $\bar{\varepsilon}=\left(\bar{\varepsilon}^{\prime}, \bar{\varepsilon}^{\prime \prime}\right)$. The elements of each of these subsets are mutually commensurable, i.e. $\bar{\varepsilon}^{\prime}=\varepsilon^{\prime} \bar{q}^{\prime}$ and $\bar{\varepsilon}^{\prime \prime}=\varepsilon^{\prime \prime} \bar{q}^{\prime \prime}$, where $\bar{q}=\left(\bar{q}^{\prime}, \bar{q}^{\prime \prime}\right)$ is a vector with all components positive. Now construct the approximate solutions singular at the points of $\Lambda^{\prime}$ as in $\S 3$, balanced exactly as before (here we use that $\left|\Lambda^{\prime}\right| \geq 2$ ). This approximate solution is of size $\varepsilon^{\prime}$ outside a fixed neighbourhood of $\Lambda^{\prime}$, and so we use a partition of unity to glue it to the approximate solution defined in each $\mathcal{T} \Lambda_{j}$.

The main step is to show that the linearization of the scalar curvature operator, $L_{\bar{\varepsilon}}$, is surjective as a map

$$
L_{\bar{\varepsilon}}: \mathcal{C}_{\nu^{\prime}, \nu^{\prime \prime}}^{2, \alpha}\left(S^{N} \backslash \Lambda\right) \oplus \mathcal{W} \longrightarrow \mathcal{C}_{\nu^{\prime}-2, \nu^{\prime \prime}-2}^{0, \alpha}\left(S^{N} \backslash \Lambda\right),
$$

at least when all the components of $\bar{\varepsilon}$ are sufficiently small. Here $1<\nu^{\prime}<2$ is the weight parameter determining growth of functions in a neighbourhood of each point of $\Lambda^{\prime}$ and $\nu^{\prime \prime}<$ $(2-N) / 2$ determines the growth near each component of $\Lambda^{\prime \prime} . \mathcal{W}$ is the same deficiency space as before. From the results of this paper, there exists an inverse for the linearization about the part of the approximate solution which is singular only at $\Lambda^{\prime}$; we denote this inverse, as well as its Schwartz kernel, by $H_{\bar{\varepsilon}^{\prime}}^{\prime}$. Let $H_{\bar{\varepsilon}^{\prime \prime}}^{\prime \prime}$ denote the right inverse, or Schwartz kernel, for the linearization about the part of the approximate solution which is singular only at $\Lambda^{\prime \prime}$, as constructed in [6]. Now let $H_{\bar{\varepsilon}}$ be a Schwartz kernel obtained by using a partition of unity (e.g. the same one as used to construct the full approximate solution) to glue together these two pieces. Then it is easy to check that

$$
L_{\bar{\varepsilon}} H_{\bar{\varepsilon}}=I+R
$$

where $R$ is of size $\max \left\{\varepsilon^{\prime}, \varepsilon^{\prime \prime}\right\}$. Clearly, then, $I+R$ is invertible, and a right inverse for $L_{\bar{\varepsilon}}$ is given by $H_{\bar{\varepsilon}}(I+R)^{-1}$. It is straightforward to verify the necessary mapping properties for this operator.

As noted above, the final step, using a contraction mapping argument to show the existence of a solution of the problem, is standard.

This completes the proof of Theorem 1 in full generality.

\section{References}

[1] D. Finn Positive solutions of $\Delta_{g} u=u^{q}+S u$ singular at submanifolds with boundary, Indiana Univ. Math. J., 43 (1994), 1359-1397.

[2] D. Finn On the negative case of the singular Yamabe problem, To appear, Jour. Geom. Anal.

[3] N. Kapouleas, Complete constant mean curvature surfaces in Euclidean three-space, Ann. Math. 131 (1990) 239-330

[4] R. Kusner, R. Mazzeo and D. Pollack The moduli space of complete embedded constant mean curvature surfaces, Geom. Func. Anal. 6 No. 1, (1996), 120-137. 
[5] R. Mazzeo, Regularity for the singular Yamabe equation, Indiana Univ. Math. J. 40 (1991), $1277-1299$

[6] R. Mazzeo and F. Pacard, A new construction of singular solutions for a semilinear elliptic equation using asymptotic analysis, Jour. Diff. Geom. 44 No. 2 (1996), 331-370.

[7] R. Mazzeo, D. Pollack and K. Uhlenbeck. Moduli spaces of singular Yamabe metrics, J. Amer. Math. Soc. 9 No. 2, (1996), 303-344.

[8] R. Mazzeo, D. Pollack and K. Uhlenbeck. Connected sum constructions for constant scalar curvature metrics, Top. Methods Nonlin. Anal. 6 No. 2 (1995), 207-233.

[9] R. Mazzeo and N. Smale, Conformally flat metrics of constant positive scalar curvature on subdomains of the sphere, J. Diff. Geom. 34 (1991), 581-621.

[10] R. C. McOwen Singularities and the conformal scalar curvature equation Geometric Analysis and P.D.E. (I. Bakelman, eds.), Marcel Decker, 1992.

[11] F. Pacard, The Yamabe problem on subdomains of even dimensional spheres, Top. Methods Nonlin. Anal. 6 No. 1, (1995), 137-150.

[12] R. Schoen, The existence of weak solutions with prescribed singular behavior for a conformally invariant scalar equation Comm. Pure and Appl. Math. XLI (1988) 317-392.

[13] R. Schoen, Variational theory for the total scalar curvature functional for Riemannian metrics and related topics Topics in Calculus of Variations; Lecture Notes in Mathematics 1365 Springer-Verlag Eds M. Giaquinta (1987) 120-154.

[14] R. Schoen and S. T. Yau, Conformally flat manifolds, Kleinian groups and scalar curvature, Invent. Math. 92 (1988), 47-72. 